\title{
Statistics and Scaling in Disordered Mesoscopic Electron Systems
}

\author{
Martin Janssen \\ Institut für Theoretische Physik, Universität zu Köln, Zülpicher Strasse 77, 50937 Köln, Germany
}

(March 21, 1997)

\begin{abstract}
This review is intended to give a pedagogical and unified view on the subject of the statistics and scaling of physical quantities in disordered electron systems at very low temperatures. Quantum coherence at low temperatures and randomness of microscopic details can cause large fluctuations of physical quantities. In such mesoscopic systems a localization-delocalization transition can occur which forms a critical phenomenon. Accordingly, a one-parameter scaling theory was formulated stressing the role of conductance as the (oneparameter) scaling variable. The localized and delocalized phases are separated by a critical point determined by a critical value of conductance. However, the notion of an order parameter was not fully clarified in this theory. The one-parameter scaling theory has been questioned once it was noticed that physical quantities are broadly distributed and that average values are not characteristic for the distributions. Based on presently available analytical and numerical results we focus here on the description of the total distribution functions and their flow with increasing system size. Still, one-parameter scaling theory does work in terms of typical values of the local density of states and of the conductance which serve as order parameter and scaling variable of the localization-delocalization transition, respectively. Below a certain length scale, $\xi_{c}$, related to the value of the typical conductance, local quantities are multifractally distributed. This multifractal behavior becomes universal on approaching the localization-delocalization transition with $\xi_{c}$ playing the role of a correlation length.
\end{abstract}

PACS numbers: 71.23.An; 71.30.+h; 72.80.Ng; 73.23.-b

Keywords: Mesoscopic Systems; Localization-Delocalization Transition;

Disordered Electrons

Typeset using REVTEX 


\section{Contents}

\begin{tabular}{ll}
\hline Introduction & 4
\end{tabular}

$\begin{array}{lll}\text { II } & \text { Mesoscopic Experiments } & 7\end{array}$

A Electronic Aharonov-Bohm-Effect . . . . . . . . . . . . . . . . 8

B $\quad$ Universal Conductance Fluctuations . . . . . . . . . . . . . . . . . . . 9

C Weak And Strong Localization . . . . . . . . . . . . . . . . . . . . 9

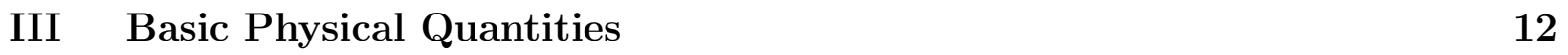

A Linear Response Quantities . . . . . . . . . . . . . . . . . . . . 12

B Relevant Scales . . . . . . . . . . . . . . . . . . . . . . . 14

\begin{tabular}{ll}
\hline IV Modeling of Mesoscopic Systems & 18
\end{tabular}

A Hamiltonian Modeling . . . . . . . . . . . . . . . . . . . . . 18

B Physical Quantities by Green's Function . . . . . . . . . . . . . . . . . . . 21

C The Statistical Problem . . . . . . . . . . . . . . . . . . . . . . . . 24

\begin{tabular}{|l|l}
\hline Scattering Matrix modeling & 26
\end{tabular}

A Quasi-One-Dimensional Conductor . . . . . . . . . . . . . . . 27

B Localization . . . . . . . . . . . . . . . . . . . . . . 30

C Networks Of Scatterers . . . . . . . . . . . . . . . 33

\begin{tabular}{|ll}
\hline VI & Statistics in Idealized Situations
\end{tabular}

A $\quad$ Localized Phase $\ldots \ldots \ldots$. . . . . . . . . . . . . . . . . . . . . . . . . . 36

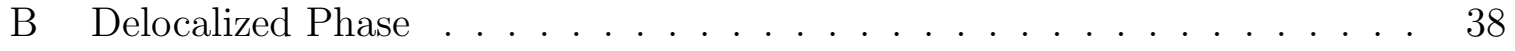

\begin{tabular}{|lll} 
VII Concept of Scaling & 41
\end{tabular}

A Relevant Scales And $\beta$-Function . . . . . . . . . . . . . . . . . . 41

B Scaling Of Typical Values . . . . . . . . . . . . . . . . . . . . . 47

C Scaling Of Distribution Functions . . . . . . . . . . . . . . . . . . . 48 


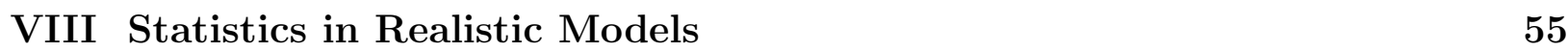

A Onset Of Broad Distributions . . . . . . . . . . . . . . . . . . 56

B Statistics in Quasi-One-Dimension . . . . . . . . . . . . . . . . . 62

C Finite Size Scaling . . . . . . . . . . . . . . . . . . . . . 70

IX $\quad$ Statistics At The Transition $\quad 72$

A Multifractality Of Critical Eigenstates . . . . . . . . . . . . . . . 72

B Local Density Of States As Order Parameter . . . . . . . . . . . . . . . . 77

C Conductance As Scaling Variable . . . . . . . . . . . . . . . . . . . . . . . 79

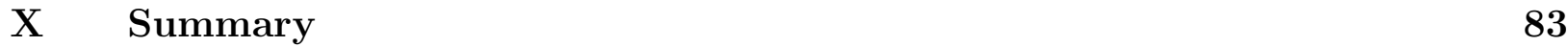

\begin{tabular}{lr}
\hline APPENDIXES & 85
\end{tabular}

\begin{tabular}{|ll}
\hline A $\quad$ Brief Account Of Linear Response Theory & 85
\end{tabular} 


\section{INTRODUCTION}

This review is about mesoscopic conductors, statistics of related physical quantities and their scaling behavior under change of the size of the conductor. A few words about the terms used here shall tell the reader what to expect.

Mesoscopic systems are intermediate between microscopic and macroscopic systems. The term goes back to van Kampen and was widely used after a work on "Physics of Mesoscopic Systems" by Imry [69]. Mesoscopic conductors contain many (e.g. 10 ${ }^{19}$ ) elementary objects like electrons and atoms. On the other hand their properties cannot be obtained by using the thermodynamic limit (volume and particle number going to infinity with fixed particle density). The thermodynamic limit is a convenient mathematical device in solid state theory if one is interested in material constants. In particular, in macroscopic conductors the conductivity, $\sigma$, is such a material constant and the conductance, $G$, is then given by Ohm's law, $G=\sigma W / L$, where $W$ is the cross section of the conductor and $L$ its length. This Ohmic behavior is to be expected once the system size (denoted by one length $L$ ) is much larger than characteristic length scales (see Fig. 1). These are (i) the kinematic scale set by the de Broglie wavelength $\lambda$ or, for low temperatures, by the Fermi wavelength $\lambda_{F}$, (ii) the elastic mean free path $l_{e}$, which is the average distance an electron travels before its initial momentum relaxes and (iii) the phase coherence length $L_{\phi}$, which is the average distance an individual electron travels before its initial and final phases become incoherent. Once the phase coherence length $L_{\phi}$ becomes larger than the system size the conductor can no longer be described by material constants. Microscopic details of the conductor will then influence even global physical quantities like conductance such that measurements yield finger prints of the mesoscopic conductor. These finger prints are due to quantum mechanical interference effects.

Since mesoscopic conductors contain many electrons one has to use a quantum statistical description for their thermodynamic and transport quantities. However, this is not the only aspect of statistics in mesoscopic conductors. Apart from the electronic degrees of freedom the conductor contains other degrees of freedom which are responsible for resistivity to applied voltages. In the simplest case these are static fields caused by impurities, vacancies and dislocations in an otherwise ideal crystal lattice. Also static inhomogeneous electric fields caused by surrounding media are responsible for resistivity. One refers to these static fields as static disorder potentials. For different realizations of a static disorder potential the global conductance of an otherwise identical mesoscopic conductor will differ - sometimes significantly. Therefore, one considers ensembles of disorder potentials characterized by a few parameters, e.g. by the average mean free path on short scales. The task of the theory is then to determine the probability distribution of physical quantities like conductance for such ensembles. Thus, on top of the quantum statistical treatment for an individual disorder potential the statistical properties of a whole ensemble of disorder potentials is addressed in 
mesoscopic physics.

Typically, the phase coherence length is of the order of a few microns for metals and semiconductors below liquid-helium temperatures. This is the reason that technological advances, starting in the 80's, were needed in order to study mesoscopic conductors systematically. We will discuss some of the fundamental experiments in section [1].

Although it is true that technological advances were needed to bring mesoscopic physics to the attention of a broader community in solid state physics, a great deal of the physics had been discussed much earlier. For example, Anderson had introduced the idea of localization already in 1958 [8]. By localization it is meant that strong disorder can trap electrons by quantum interference to a finite region such that the conductor actually behaves as an insulator. Also, Landauer had presented a formula in 1957 [84] that describes the conductance of a phase coherent conductor in terms of pure quantum mechanical transmission amplitudes, and a similar important approach to the conductance of mesoscopic systems goes back to works in 1972 by Edwards and Thouless [45].

The works by Edwards and Thouless [45] and by Wegner [131] form the starting point of the scaling theory for mesoscopic conductors. Later in 1979 it was formulated in a simple and predictive way by Abrahams, Anderson, Licciardello and Ramakrishnan [1]. The scaling theory for disordered mesoscopic conductors is based on the assumption that the transport properties of systems probed at scales much larger than the mean free path should become insensitive to the microscopic origin of the disorder. The scaling theory states that once we know the conductance for a given system size we can obtain the conductance for an even larger system from a universal flow equation. Now, since we have already pointed out that the conductance is a random variable which depends in a complicated manner on the particular disorder realization this assumption needs further explanation. As has been stressed by Anderson et al. [9] and later by Shapiro [115,116] it can be correct only in a probabilistic sense. The whole probability distribution of the conductance approaches a universal distribution function which depends only on very few characteristic system parameters.

The scaling theory leads to a classification of mesoscopic electron systems into three classes: depending on the initial values of the characteristic system parameters (called scaling variables) for a system size $L_{0}$ the system will flow under increase of system size to an insulating state (localized phase), or to an conducting state (delocalized phase), or it stays scale independent at a critical state. Thus a disorder induced transition from a localized to a delocalized phase can occur which resembles a critical phenomenon. This transition is frequently denoted as Anderson transition or metal-insulator transition (MIT). To distinguish this disorder induced MIT from other MITs occurring in solid state physics we adopt the notion of localization-delocalization (LD) transition. Accordingly, the critical point is referred to as the LD transition point.

The central aim of this article is to provide a unique picture about the LD transition in mesoscopic disordered electron systems. To achieve this aim we will introduce the basic 
physical quantities, scales and theoretical models and rely on simple calculations avoiding involved technical details. We stress general concepts and methods such as quantum composition rules for conductors, Fokker Planck equations, and scaling laws for distribution functions. Important results that are based on technically more elaborate methods will only be quoted without detailed description. Nevertheless, we hope that the present article will help to find a way through the original literature.

A number of reviews and books exist on related subjects discussed in this article. The following short list shall help for further reading. The review by Y. Imry [69] is an introduction to mesoscopic physics. A review on the subject of mesoscopic transport that contains also information about the technological aspect of devices was written by Beenakker and van Houten [15]. The book by Datta [38] on "Electronic Transport in Mesoscopic Physics" covers many subjects that have proved to be relevant in the field. As review on localization and scaling theory for mesoscopic systems we recommend the ones by Lee and Ramakrishnan [88] and by Kramer and MacKinnon [79]. The book by Al'tshuler and Lee [7] on "Mesoscopic Phenomena in Solids" contains review articles by inventors in the field.

The present article is organized as follows. In Sect. [1] fundamental experiments will be discussed. The electronic Aharonov-Bohm-Effect makes the definition of mesoscopic conductors more precise. Universal conductance fluctuations demonstrate the finger-print character of mesoscopic conductance measurements and experiments on weak and strong localization point to the necessity of a critical phenomenon description of the LD transitions. Basic physical quantities are the linear response quantities such as conductance. These and the corresponding relevant energy and length scales are the subject of Sect. [II. To discuss the statistical problem inherent in the problem of mesoscopic conductors we introduce Hamiltonian models in Sect. $\mathbb{Z}$. There we will see that the Green's function contains the essential information. Only the asymptotic behavior of the Green's function is needed for describing global conductances resulting in a scattering theoretical formulation of mesoscopic conductors (Sect. $\nabla$ ). This point of view has been particular useful in describing strong localization in so-called quasi-one-dimensional systems. However, the scattering theoretical modeling is not restricted to this case but can be extended to more general geometries by considering networks of scattering matrices. After the basic quantities and tools have been presented we discuss the statistical properties of mesoscopic conductors in the idealized localized and delocalized phase, respectively (Sect. VI). To prepare for the statistics in more realistic systems we introduce the concept of the scaling theory in Sect. VTT. The flow of the relevant scaling variables is controlled by so-called $\beta$-functions from which a critical exponent for the LD transitions can be obtained. It describes how the correlation length of this critical phenomenon diverges on approaching the LD transition point. Based on a pedagogical model, introduced by Shapiro (cf. [116]), we emphasize that typical values of the conductance distribution can be good candidates for scaling variables. We pay special attention to the statistics of local quantities (like the local density of states) that show power 
law scaling in non-localized phases. We come to the conclusion that, at the LD transition, a spectrum of power law exponents (called multifractal spectrum) is needed in order to describe the distribution. The more realistic models are the subject of Sect. VIII. The onset of broad distributions characterizing global and local quantities at large, but finite, values of typical conductance leaves open questions about their interpretation. To clarify some of them the conductance distribution in an idealized quasi-one-dimensional geometry is studied. The idealization is due to the assumption of absence of transversal diffusion in such systems. The calculations give useful insight into the physics of universal conductance fluctuations and lead to the interpretation that the onset of broad tails in the conductance distribution is intimately connected to the critical regime of the LD transition. Such regime, as well as long tails in the conductance distribution, is absent in the idealized quasi-onedimensional conductor. Giving up the idealization, one can study the LD transition in quasione-dimensional systems by the so-called finite size scaling method. Due to the complexity of calculations for non-ideal quasi-one-dimensional systems the finite size scaling method is often restricted to numerical calculations. However, such calculations are very successful and provide a lot of insight into the physics as well as quantitative results for universal properties. The investigation of statistics and scaling of mesoscopic disordered electron systems close to the LD transition in Sect. IX] reveals the multifractal properties of electronic states and opens the possibility to consider the local density of states as an appropriate order parameter field for the LD transition. The typical value, as given by the geometric mean, is the global order parameter. Similarly, we collect arguments that allow to view the typical conductance as the only relevant scaling variable. Our conclusions are summarized in Sect. $\mathrm{X}$ and a brief account of linear response theory is given in the appendix Sect. A.

\section{MESOSCOPIC EXPERIMENTS}

In this section we will discuss some fundamental mesoscopic experiments without paying much attention to historical order and completeness. Rather, we wish to give motivation to the following sections which are based on a theoretical physicists point of view.

The electronic Aharonov-Bohm-effect can be used for a definition of mesoscopic conductors. It is our starting point of investigations. It points to the necessity for a quantum mechanical description of transport. The need to describe physical quantities in terms of distribution functions rather than in terms of material constants became evident after the discovery of universal conductance fluctuations. That one conductor can behave both, as a perfect conductor and as a perfect insulator, when changing system parameters is the insight coming from experiments on weak and strong localization. Weak localization refers to a situation where the system is metallic, but the conductivity is reduced as compared to its classical value. This behavior is interpreted as a precursor of the strong localization phenomenon where the system is insulating due to spatial localization of electronic states. 
Weak localization effects have been found in various materials. The experimental verification of strong localization is most clear-cut in the quantum Hall effect, and first experimental investigations of fluctuations at the LD transition point have been performed.

\section{A. Electronic Aharonov-Bohm-Effect}

A ring-shaped conductor shown schematically in Fig. 2 with wire thickness of $\approx 40 \mathrm{~nm}$ and a diameter of $\approx 820 \mathrm{~nm}$ that was etched out of a high quality polycrystalline gold film of thickness $\approx 40 \mathrm{~nm}$ was used to perform the first realization of the most fundamental experiment in mesoscopic physics, the electronic Aharonov-Bohm-Effect. At temperatures less than $1 \mathrm{~K}$ the resistance oscillates as a function of the magnetic flux enclosed by the ring [129]. Figure 3 shows the result for the resistance oscillations found in a ring-shaped conductor fabricated in high mobility GaAs-Al GaAs heterostructures performed by Timp et al. [124]. Here the diameter of the ring is $2 \mu \mathrm{m}$. The period in the magnetic field $(\approx 5 \mathrm{mT})$ corresponds to a periodicity in the magnetic flux enclosed by the ring of one flux quantum $h / e$ where $-e$ is the elementary charge of an electron and $h$ is Planck's constant. The qualitative explanation of the oscillations relies on the concept that electrons in a magnetic field $\mathbf{B}=$ curl $\mathbf{A}$, where $\mathbf{A}$ is the vector potential, pick up a quantum mechanical phase,

$$
\varphi=\frac{e}{\hbar} \int_{C} \mathbf{A}(\mathbf{r}) \cdot d \mathbf{r},
$$

when they move along a classical path $C$ that connects two points in space. Now, for an electron coming from the current source the path splits up into the upper and lower branch of the ring and the phase difference $\varphi_{u}-\varphi_{l}$ between both paths is given by the total magnetic flux $\phi$ enclosed by the ring

$$
\varphi_{u}-\varphi_{l}=\frac{e}{\hbar} \oint \mathbf{A}(\mathbf{r}) d \mathbf{r}=\frac{e}{\hbar} \phi
$$

In terms of the the flux quantum $\phi_{0}:=h / e$ the phase difference is $2 \pi \phi / \phi_{0}$. Taking for granted that the resistance is sensitive to quantum interference of electronic waves the periodicity of the resistance is given by $\phi_{0}$. The electronic Aharonov-Bohm-Effect demonstrates that the electrons are to be treated quantum mechanically along the ring and that their initial phase is not randomized before leaving the ring. This phenomenon is called phase coherence and the Aharonov-Bohm-Effect gives rise to an operative definition of the phase coherence length $L_{\phi}$ (see also Sect. [IIB). On increasing the temperature or the diameter of the ring the phase coherence gets lost and the Aharanov-Bohm effect disappears. The system size at which the Aharanov-Bohm effect (gradually) disappears quantifies the temperature dependent phase coherence length $L_{\phi}$. The disappearance of the Aharanov-Bohm effect is caused by an increasing number of inelastic scattering events which destroy the phase coherence. The average distance of inelastic scattering events can be identified with $L_{\phi}$. 


\section{B. Universal Conductance Fluctuations}

Measurements of conductance in mesoscopic systems that have been performed since the 80 's, showed sample specific, reproducible, statistical fluctuations when system parameters like Fermi energy, magnetic field or disorder configurations were changed [130]. In Figure 4 the results of a recent experiment are shown. Gold-nanowires of very high purity with cross-section $30^{2} \mathrm{~nm}^{2}$ and lengths between 400 and $1000 \mathrm{~nm}$ were used. The conductance $G$ was measured as a function of applied magnetic field below $16 \mathrm{~T}$ in steps of $1 \mathrm{mT}$ at $60 \mathrm{mK}$. Although the average conductance varies from $\approx 3000 e^{2} / h$ (for $L=400 \mathrm{~nm}$ ) to $\approx 1400 e^{2} / h$ (for $L=1000 \mathrm{~nm}$ ), the variance of the conductance stayed constant and was $\approx 0.1\left(e^{2} / h\right)^{2}$. This phenomenon is referred to as universal conductance fluctuations (UCF). In a mesoscopic conductor with high conductance (in atomic units $e^{2} / h$ ) the conductance fluctuations are reproducible finger-prints of the conductor. However, the variance turns out to be approximately a constant

$$
\operatorname{var}(G)=\text { const. } \sim \mathcal{O}(1) \times\left(e^{2} / h\right)^{2}
$$

Two aspects of UCF are interesting: reproducibility and universality. That the fluctuations are reproducible can be understood again by a semiclassical description of conducting electrons. Consider electrons moving along classical paths between elastic collisions, but having a quantum mechanical phase attached. Since the phase information is not lost over distances of the phase coherence length (mesoscopic regime) a sample specific interference pattern arises. The interference pattern will be altered e.g. by applying a magnetic field. This gives rise to reproducible conductance fluctuations. The fact that the order of magnitude of these fluctuations turns out to be independent of the average conductance and does only depend weakly on dimensionality and symmetry properties of the conductor is, however, not easy to understand without having a transport theory of mesoscopic conductors. A striking consequence of the phenomenon is that mesoscopic conductors can not be characterized by an average conductivity being a material constant, even if the system size $L<L_{\phi}$ becomes very large. Conductivity $\sigma$ is related to conductance by Ohm's law. For a cubic geometry

the conductance behaves as $G=\sigma L^{d-2}$. For UCF the relative fluctuations of conductance are $\delta G / G \propto L^{2-d}$ which is in contrast to the classical behavior $\delta G / G \propto L^{-d / 2}$. Thus, even in $3 \mathrm{D}$ metallic systems the relative fluctuations are much stronger than $1 / \sqrt{\text { volume. }}$

\section{Weak And Strong Localization}

Early measurements of conductivity of amorphous Silicon [19] showed that, at low enough temperatures, the temperature behavior of conductivity follows Mott's $T^{-1 / 4}$-law,

$$
\sigma(T)=\sigma_{0} \exp \left(-\left(T_{0} / T\right)^{1 /(d+1)}\right) \quad \text { for } d=3,
$$


over two orders of magnitude with some constants $T_{0}$ and $\sigma_{0}$ (see Fig. 5). This behavior of the conductivity could be understood when assuming that the transport is mediated by phonon assisted hopping processes between localized states [100]. Localized states are spatially localized to some finite volume within the conductor. The finite volumes are characterized by a localization radius $\xi_{r}$. The phonon assisted hopping is possible because localized states can be energetically close to each other if the corresponding localization centers are separated by distances much larger than the localization radius. The observation of Mott's law made it clear that electronic states can indeed be localized by disorder which has been pointed out by Anderson [8] much earlier. The effect is referred to as strong localization since the localization radius is smaller than system size.

A further experimental hint to localization came from weakly disordered metallic films at low temperatures. They showed a logarithmic increase of resistance when the temperature is decreased (see Fig. 6). Although the system behaves metallic with large conductance the conductance was lower than its classical value. The effect was interpreted as being due to quantum interference corrections to classical transport and is referred to as weak localization. In weak localization the localization length is larger than system size but the effect is interpreted as a precursor of strong localization, i.e. under further increase of system size (while still being mesoscopic, i.e $L<L_{\phi}$ ) the system would show strong localization. This is, of course, in practice very hard to realize when localization lengths are larger than microns.

The weak localization effect (predicted by Gor'kov et al. [59]) can be explained by the phenomenon of enhanced backscattering due to quantum interference. It relies on the fact that the quantum coherent probability of the sum of two probability amplitudes $|A+A|^{2}=$ $4|A|^{2}$ is two times larger than the incoherent sum of the corresponding probabilities, $|A|^{2}+$ $|A|^{2}=2|A|^{2}$. Consider the chance for an electron traveling in a phase coherent conductor (see Fig. 1) to return back to some point within the conductor. In Fig. 7 a possible path is drawn for such a path (straight line). If the motion is time reversal symmetric also the time reversed path (broken line) is possible. The amplitudes of both paths add coherently in a semiclassical calculation of the return probability. Thus, the quantum mechanical return probability is twice the classical value. Without explicitly calculating the conductivity it is obvious from this observation that the conductivity must be reduced as compared to its classical value.

The coherence is limited by the phase coherence length $L_{\phi}$ which varies as some inverse power of temperature, $L_{\phi}(T) \propto T^{-p}$ For system sizes $L$ larger than the phase coherence length $L_{\phi}(T)$ the logarithmic corrections to the classical conductivity could be interpreted as being due to the quantity $-\ln L_{\phi} / l_{e}$ where $l_{e}$ is the average elastic mean free path.

Magnetic fields destroy the time reversal symmetry of the electronic motion and thus the weak localization effect vanishes (see upper set of Fig. 8) A very interesting effect is due to spin-orbit scattering processes that are time reversal symmetric. In this case the time 
reversed path, however, gives rise to destructive interference in the return probability. As a result the sign of the conductance corrections is reversed (see lower set of Fig. 8) and the enhancement of conductivity above its classical value is denoted as weak anti-localization (first predicted by Hikami et al. [63]).

Weak localization experiments are an important tool e.g. to determine the phase coherence length $L_{\phi}$ and the spin-orbit scattering length. For more details we refer to the review by Bergmann [18].

To reach experimentally the regime of strong localization where the localization radius is less than the system size $L<L_{\phi}$ one has to use strongly disordered systems or work in energy regions with very low density of states. Localization-delocalization (LD) transitions have been observed in several experiments (for reviews see [104,113]). Figure 9 shows the finding of an experiment by Stupp et al. [122] indicating that the conductivity vanishes on approaching a critical charge carrier concentration. A major problem in comparing theoretical models with the experiments comes from the fact that in the low density of states regimes Coulomb interaction between electrons becomes essential, but most theoretical models are based on an independent electron picture.

The most clear-cut experiment for the occurrence of LD transitions is the quantum Hall effect discovered by von Klitzing [76]. The quantum Hall effect that occurs in twodimensional electron gases in the presence of strong perpendicular magnetic fields is characterized by a step-function like behavior of the Hall conductivity $\sigma_{H}$ as a function of the so called filling factor (a dimensionless quantity proportional to carrier concentration and inverse magnetic field) and by a vanishing dissipative conductivity $\sigma$ in the Hall plateau regimes (see Fig. 10; Note, that for non-vanishing Hall conductivity a vanishing dissipative conductivity causes also a vanishing longitudinal resistivity.). The peaks in the dissipative conductivity as a function of the filling factor have a clear interpretation in terms of LD transitions. However, no metallic phase exists. All states, except for those at critical fillings where the localization radius is larger than system size, are strongly localized. The systems showing the quantum Hall effect (quantum Hall systems) are well suited to study the properties of critical states. Recently, in an experiment by Cobden and Kogan [35] it was possible to extract the whole conductance distribution at criticality for a truly mesoscopic quantum Hall system. The main finding was a conductance distribution which is independent of system size (within the mesoscopic regime), almost uniform between 0 and $e^{2} / h$. This means in particular that the fluctuations are of the same order as the average value. Thus, fluctuations in the conductance can become much stronger than one could expect from classical transport theory where the relative fluctuations scale like the square root of the inverse volume. 


\section{BASIC PHYSICAL QUANTITIES}

This section prepares for the discussion of transport theory in mesoscopic electron systems. We introduce the basic physical quantities of disordered mesoscopic systems for which the phase coherence length is larger than the system size. They are introduced here on a phenomenological level as response quantities to applied fields. The modeling of mesoscopic systems and the tools to calculate these quantities is the subject of the following Sect. IV (and the appendix A). The basic physical quantities are the thermodynamic density of states and the transport quantities resistance, conductance and diffusion. Related physical scales are thermodynamic and kinematic scales like level spacing, Fermi energy, Fermi wavelength, magnetic length and transport scales like Thouless energy, elastic mean free path and the localization length.

\section{A. Linear Response Quantities}

The thermodynamic density of states

$$
\rho(\mu)=\frac{\partial n}{\partial \mu}
$$

determines the change of the global particle density $n$ with respect to a change in the chemical potential $\mu$. The local thermodynamic density of states $\rho(\mathbf{r} ; \mu)$ is defined similarly when replacing $n$ by the local particle density $n(\mathbf{r})$.

In charge transport one considers a stationary though non-equilibrium situation. Global transport quantities are the resistances in a multi-probe setup

$$
\mathcal{R}_{k l, m n}=\frac{U_{m}-U_{n}}{I}, \quad I=I_{k}=-I_{l}, \quad I_{i \neq\{k, l\}}=0
$$

where probes are characterized by electro-chemical potentials $U_{i}$. The current $I$ is driven from the current source (probe $k$ ) to the current sink contact (probe $l$ ) (cf. Fig. 11).

The resistances $\mathcal{R}_{k l, m n}$ are determined by the conductance coefficients $G_{k n}$ [20]

$$
I_{k}=\sum_{n} G_{k n}\left(U_{k}-U_{n}\right)
$$

With the condition of total current conservation $\sum_{k} I_{k}=0$ the conductance coefficients $G_{k n}$ fulfill the relations 1

\footnotetext{
${ }^{1}$ Equations (6, (6) can also be considered for time dependent transport with the quantities $U_{m}$, $I_{k}$ being the Fourier components for frequency $\omega$. In that case $\mathcal{R}_{k l, m n}$ and $G_{k m}$ will depend on $\omega$,
} 


$$
\sum_{k} G_{k n}=\sum_{n} G_{k n}=0
$$

In a two-probe geometry the transport is determined by the two-probe conductance $G$ (Ohm's law)

$$
G=I / U=R^{-1}
$$

where $U=U_{1}-U_{2}$ and $G=G_{12}$ and $R=\mathcal{R}_{12,12}$.

A full description of the local charge transport is possible with the help of the conductivity tensor, $\sigma_{\mu \nu}\left(\mathbf{r}, \mathbf{r}^{\prime} ; \omega\right)$,

$$
j_{\mu}(\mathbf{r})=\int d^{d} r^{\prime} \sum_{\nu} \sigma_{\mu \nu}\left(\mathbf{r}, \mathbf{r}^{\prime} ; \omega\right) E_{\nu}\left(\mathbf{r}^{\prime}\right)
$$

relating local electric field, $\mathbf{E}(\mathbf{r})$, to the local current density, $\mathbf{j}(\mathbf{r})$. Both can here be considered as the Fourier components for frequency $\omega$ of the corresponding time-dependent quantities $\left(\sim e^{-i \omega t}\right)$.

Local currents can be also generated by charge density gradients under conditions of local equilibrium, i.e. the chemical potential $\mu$ is a function of position. The relevant transport coefficient in this case is the diffusion function, $D_{\mu \nu}\left(\mathbf{r}, \mathbf{r}^{\prime} ; \omega\right)$,

$$
j_{\mu}(\mathbf{r})=-\sum_{\nu} \int d^{d} r^{\prime} D_{\mu \nu}\left(\mathbf{r}, \mathbf{r}^{\prime} ; \omega\right) \frac{\partial}{\partial r_{\nu}^{\prime}} q\left(\mathbf{r}^{\prime}\right)
$$

This name is justified since the continuity equation between current and charge density, $q(\mathbf{r}), \operatorname{div} \mathbf{j}(\mathbf{r})=i \omega q(\mathbf{r})$ tells that $q(\mathbf{r})$ obeys a non-local diffusion equation

$$
-i \omega q(\mathbf{r})=\sum_{\mu \nu} \int d^{d} r^{\prime} \frac{\partial}{\partial r_{\mu}} D_{\mu \nu}\left(\mathbf{r}, \mathbf{r}^{\prime} ; \omega\right) \frac{\partial}{\partial r_{\nu}^{\prime}} q\left(\mathbf{r}^{\prime}\right)
$$

Under the conditions of local equilibrium the charge density can be related to the gradient of the chemical potential via the thermodynamic charge response, $\Pi\left(\mathbf{r}, \mathbf{r}^{\prime}\right)=e^{2} \delta n(\mathbf{r}) / \delta \mu\left(\mathbf{r}^{\prime}\right)$,

$$
\frac{\partial}{\partial r_{\mu}} q(\mathbf{r})=(-e)^{-1} \int d^{d} r^{\prime} \Pi\left(\mathbf{r}, \mathbf{r}^{\prime}\right) \frac{\partial}{\partial r_{\mu}^{\prime}} \mu\left(\mathbf{r}^{\prime}\right) \text {. }
$$

Since the linear response quantities conductivity and diffusion belong to the same system and describe currents in response to local electric and chemical potential gradients, respectively, they can be related by identifying the currents and driving fields. This constitutes a generalization of the important Einstein relation between conductivity and diffusivity,

too. The total current conservation and Eq.(8) will still be valid as long as electric fields which are generated when applying voltages to the system (including the coherent probe region) have their sources and sinks within the system (see e.g. [21]). 


$$
\sigma_{\mu \nu}\left(\mathbf{r}, \mathbf{r}^{\prime} ; \omega\right)=\int d^{d} r^{\prime \prime} D_{\mu \nu}\left(\mathbf{r}, \mathbf{r}^{\prime \prime} ; \omega\right) \Pi\left(\mathbf{r}^{\prime \prime}, \mathbf{r}^{\prime}\right)
$$

Equation (10) reduces to Ohm's law for spatially averaged currents and electric fields

$$
j_{\mu}=\sum_{\nu} \sigma_{\mu \nu}(\omega) E_{\nu}
$$

and for the global conductivity tensor, $\sigma_{\mu \nu}$, Eq. (13) reduces to the global Einstein relation between conductivity and diffusion

$$
\sigma_{\mu \nu}(\omega)=e^{2} D_{\mu \nu}(\omega) \frac{\partial n}{\partial \mu} .
$$

In the following we will concentrate on the d.c. limit of transport $(\omega \rightarrow 0)$ and on a longitudinal (dissipative) component of the conductivity tensor, say $\sigma:=\sigma_{x x}$. In the case where off-diagonal components of conductivity vanish, e.g. in the absence of magnetic field, the two-probe conductance $G$ of a system with box geometry (characterized by length $L$ and cross section $L_{t}^{d-1}$, see Fig. 12) is related to the conductivity $\sigma$ by

$$
G=\sigma L_{t}^{d-1} / L
$$

Equation (17) is also referred to as Ohm's law. Whenever off-diagonal components of conductivity are present Eq. (17) refers to the dissipative conductance which can be measured in an appropriate four-probe setup. We stress that the global conductivity is treated here as a spatial average over the conductivity tensor, $\sigma=\int d^{d} r \int d^{d} r^{\prime} \sigma\left(\mathbf{r}, \mathbf{r}^{\prime}\right) /\left(L L_{t}^{d-1}\right)$, and may depend on $L, L_{t}$.

\section{B. Relevant Scales}

The Einstein relation, Eq. (16), and Ohm's law, Eq. (17) allow for a very interesting interpretation of the conductance measured in atomic units $e^{2} / h$. Defining a diffusion time $t_{D}$ by $t_{D}:=L^{2} / D$ and a quantum mechanically related energy scale

$$
E_{\mathrm{Th}}:=h / t_{D}
$$

called Thouless energy, and the thermodynamic level spacing, $\Delta(\mu)$, by

$$
\left(L L_{t}^{d-1}\right) \frac{\partial n}{\partial \mu}=:(\Delta(\mu))^{-1}
$$

we obtain the Thouless formula [123] for the dissipative conductance, $G=\left(e^{2} / h\right) g$,

$$
g=E_{\mathrm{Th}} / \Delta
$$


This means that the dimensionless conductance, $g$, can be expressed as the ratio of two characteristic energy scales of the problem: a transport related energy scale $E_{\mathrm{Th}}$ and a thermodynamically defined energy scale $\Delta$. As we will see later this expression will help a lot in classifying mesoscopic electron systems.

So far we have not introduced any particular dynamical properties of the electron system. In a linear response treatment as outlined in the appendix Sect. A thermodynamic as well as dynamic response quantities can be calculated by appropriate correlation functions. In a first attempt we make a phenomenological ansatz to describe the global dissipative conductivity $\sigma$ thereby making contact with standard macroscopic transport theory.

In the absence of magnetic fields and in the presence of static disorder one can make the plausible assumption that an electron starting with velocity $\mathbf{v}$ will decelerate owing to scattering by impurities. This process can be modeled in the time dependent velocity correlator (see Eq. (A4, A8)

$$
\left\langle v_{\nu} ; v_{\mu}(t)\right\rangle=v_{0}^{2} e^{-t / \tau} \delta_{\mu \nu}
$$

where $\tau$ is a phenomenological momentum relaxation time and $v_{0}^{2}$ the static velocity autocorrelator for a given direction and serves also as a measure of the kinetic energy of the initial state. With the help of the Kubo relation, Eq. (A11), this correlator is found to be

$$
v_{0}^{2}=\frac{N}{\beta m}
$$

( $N$ being the number of electrons, $\beta$ the inverse temperature $1 / k_{B} T$ and $m$ being the electrons mass). The global conductivity is proportional to the Laplace transform of the velocity correlator (see Eq. (A19)) which yields the Drude formula for the d.c. conductivity

$$
\sigma_{x x}=\frac{e^{2} n \tau}{m}, \quad \sigma_{y x}=0
$$

For non-interacting electrons, at $T=0$, Eq. (23) can be interpreted as

$$
\sigma_{x x}\left(E_{\mathrm{F}}\right)=e^{2} n\left(E_{\mathrm{F}}\right) \tau\left(E_{\mathrm{F}}\right) / m=e^{2} \rho\left(E_{\mathrm{F}}\right) \frac{v_{F}^{2} \tau\left(E_{\mathrm{F}}\right)}{d}
$$

where $E_{\mathrm{F}}$ is the Fermi energy, $v_{F}=\sqrt{(2 / m) E_{\mathrm{F}}}$ the Fermi velocity and $\rho\left(E_{\mathrm{F}}\right)$ the density of states.

In a finite system of volume $L^{d}$ the density of states defines the level spacing

$$
\Delta=\left(\rho L^{d}\right)^{-1}
$$

which is the smallest quantum kinematic energy scale of the system.

The Fermi velocity sets a quantum kinematic length scale, the Fermi wavelength

$$
\lambda_{F}=\frac{h}{v_{F} m}
$$


The momentum relaxation time is equivalent to an elastic mean free path, $l_{e}$, that describes a typical distance between elastic collisions

$$
l_{e}=v_{F} \tau \text {. }
$$

The corresponding diffusion constant follows from the Einstein relation, Eq.(16),

$$
D=\frac{v_{F} l_{e}}{d}
$$

Assuming that in a large but finite system the diffusion constant is indeed a constant the Thouless energy, Eq. (18), and the conductance, Eq. (19), can be determined as

$$
E_{\mathrm{Th}}=\frac{h D}{L^{2}}, \quad g=\frac{S_{d}}{d}\left(\frac{L}{\lambda_{F}}\right)^{d-2} \frac{l_{e}}{\lambda_{F}} .
$$

Here $S_{d}$ is a geometrical number, the surface area of the unit sphere in $d$ dimensions, e.g. $2 \pi$ in $2 \mathrm{D}$. The situation with $l_{e} \gg \lambda_{F}$ corresponds to large $g$ and will be denoted as weak scattering case.

Within macroscopic transport theory the transport is determined by material constants, e.g. the diffusion constant, and the basic task is to calculate them from microscopic models. The situation changes when the system becomes smaller than the phase coherence length $L_{\phi}$.

Consider a given conducting system with fixed geometry and at fixed temperature. Imagine that we can pierce a hole in the conductor and put a magnetic flux through it. As quantum mechanics tells, such flux can only influence the phase of the electrons wave functions. In case that one observes periodic oscillations in the resistances when changing the flux over several flux quanta, $h / e$, (electronic Aharonov-Bohm effect, see Sect.IIA) the electronic system is mesoscopic, i.e. the electronic wave functions are phase coherent over the entire conducting system. As soon as the charge carrying wave packets experience scattering processes with dynamical degrees of freedom, e.g. phonons, that can dissipate energy from the electronic system the phase coherence will be destroyed and the electronic AharonovBohm effect gets lost. It is worth noticing that elastic scattering cannot destroy the phase coherence. Thus, the criterion for an electron system to be mesoscopic is set by the phase coherence length $L_{\phi}$ being larger than the system size $L$,

$$
L_{\phi} \geq L
$$

Here $L_{\phi}$ can be considered as a typical distance before the charge carrying states experience an inelastic scattering event. It is evident that $L_{\phi}$ will usually decrease with increasing temperature. In practice one has to go below liquid Helium temperatures and use microstructured electron systems to reach the mesoscopic regime.

The main consequence of the phase coherence in mesoscopic conductors is the fact that quantum interference plays a dominant role for physical quantities to be observed. As 
we have already seen in Sec. [1] it can cause two important effects: unexpectedly large fluctuations and localization.

The first effect can be qualitatively described as follows. A wave packet entering the mesoscopic conductor will be scattered by some elastic collisions. After a while it will form a complicated interference pattern. If one changes an external parameter only slightly, e.g. magnetic field, one has to be aware of drastic effects on the measured physical quantity since the interference pattern changes. A similar effect can be caused by a slight modification in the arrangement of elastic scatterers. At least, one cannot expect that a measured physical quantity is close to an average value obtained from a large number of measurements under slightly different conditions. Instead, the quantities will show fluctuations which can become unexpectedly large. However, such fluctuations are reproducible. The magnitude of mesoscopic fluctuation effects will depend on system specific parameters. This will be the topic of Secs. VII,VII], [XX. What can be learned by this reasoning is that a characterization of a mesoscopic sample is incomplete unless the whole distribution function of the physical quantity of interest is obtained. It is no longer possible to describe it by material constants (see Sect. [IB). This insight forms the motivation to ask for the statistical properties of mesoscopic electron systems.

The second effect, localization, was pointed out by Anderson in a by now famous paper [8]. He demonstrated in a model calculation that the conductance of a phase coherent disordered (mesoscopic) system can decrease exponentially with increasing system size. Denoting $L$ as the linear system size, the conductance can scale as

$$
g(L) \propto \exp (-2 L / \xi)
$$

where $\xi$ is called the localization length A. The origin of this phenomenon lies in the multiple interference of the electrons wave packet after several elastic collisions that can lead to destructive interference such that the wave packet is bound to a finite region, the localization volume, with exponentially decreasing amplitude outside of this region. For system sizes that are several times larger than the localization length the conductor actually behaves as an insulator. The effect becomes even more interesting since it is possible that one system can change from the insulating, localized, phase to a conducting, delocalized, phase when parameters are changed continuously. Such parameters can be the degree of disorder, electron density or external fields like pressure, electric or magnetic field (see Sect. IIO). Thus, mesoscopic systems can undergo a disorder induced metal-insulator transition which we called localization-delocalization transition (LD transition).

\footnotetext{
${ }^{2}$ The factor of 2 is due to conventions which will be discussed later.
} 


\section{MODELING OF MESOSCOPIC SYSTEMS}

In this section we introduce model systems appropriate for disordered mesoscopic systems and describe how to obtain relevant physical quantities. Since we do not want to describe coupling of electrons to dynamic degrees of freedom that can dissipate energy from the electronic system we ignore from the very beginning electron-phonon interactions and restrict ourself to zero temperature, unless otherwise stated. In addition, we will treat electrons mainly as independent additive systems characterized by effective mass, only. This is a drastic restriction and it is in general not justified to incorporate electron-electron interactions into such effective one-particle models. However, with one-particle models one can already gain some insight into the mechanism of LD transitions and fluctuation effects. The interplay of disorder and electron-electron interaction forms a challenging research field which has still to be developed. Furthermore, internal degrees of freedom of the electrons will be ignored to a large extent, leading to models of non-interacting spin-less fermions in the presence of static disorder potentials.

\section{A. Hamiltonian Modeling}

Independent electrons of effective mass $m$ moving in a random one-particle potential $V(\mathbf{r})$ are described by the Hamiltonian

$$
H=\frac{(\mathbf{p}+e \mathbf{A})^{2}}{2 m}+V(\mathbf{r})
$$

where the kinetic momentum $\mathbf{p}+e \mathbf{A}$ may include magnetic fields $\mathbf{B}=$ curl $\mathbf{A}$. Randomness of the disorder potential has to be specified by a distribution function. It is often assumed that the essential parameters of the distribution are contained in the first and second cumulant, i.e. a Gaussian distribution, fixed by the mean value $\langle V(\mathbf{r})\rangle$ and a finite range correlation function $\left\langle V(\mathbf{r}) V\left(\mathbf{r}^{\prime}\right)\right\rangle$, will be general enough to cover the essential physics. It is reasonable to assume that neither specific points, nor specific directions are preferred by the whole ensemble of potentials. Then one can characterize the ensemble by two parameters, a potential correlation strength, $V_{0}$, and a potential correlation length, $l_{V}$,

$$
\begin{aligned}
\langle V(\mathbf{r})\rangle & \equiv 0 \\
\left\langle V(\mathbf{r}) V\left(\mathbf{r}^{\prime}\right)\right\rangle & =V_{0}^{2} \exp \left(-\left|\mathbf{r}-\mathbf{r}^{\prime}\right|^{2} / l_{V}^{2}\right)
\end{aligned}
$$

In case that $l_{V}$ can be considered to be smaller than other relevant microscopic scales, it can be set to zero, leading to a Gaussian white-noise potential

$$
\left\langle V(\mathbf{r}) V\left(\mathbf{r}^{\prime}\right)\right\rangle=\gamma^{2} \delta\left(\mathbf{r}-\mathbf{r}^{\prime}\right)
$$


where $\gamma^{2}$ corresponds to $\left(\sqrt{\pi} l_{V}\right)^{d} V_{0}^{2}$. Within the Gaussian white-noise potential model the disorder is characterized by one parameter, $\gamma^{2}$, of dimension [Energy ${ }^{2}$ Volume]. We will see later, Eq. (59), how this parameter corresponds to an elastic mean free path, $l_{e}$.

A homogeneous magnetic field pointing in a fixed direction, say $\mathbf{B}=(0,0, B)$, introduces a quantum mechanical length scale, the magnetic length $l_{B}$ defined by the size of an area which is penetrated by one flux quantum $h / e$

$$
l_{B}=\sqrt{\hbar /(e B)} .
$$

The magnetic length comes along with chirality due to the axial vector character of magnetic field. As long as the magnetic length is larger than the actual system size, the effect of the magnetic field on the classical paths of electrons can be neglected and it will only influence the phases of wave functions. As soon as the magnetic length is of the order of the mean free path $l_{B} \sim l_{e}$ the dynamics is, to a large extent, determined by the magnetic field and we refer to this situation as strong magnetic field case.

A simple modeling to account for possible spin-orbit scattering events that can flip the electron's spin is given by the addition of

$$
\mathbf{S} \cdot\left(\operatorname{grad} U_{\mathrm{SO}} \times \mathbf{p}\right)
$$

to the Hamiltonian in Eq. (32) [46,52]. Here $\mathbf{S}$ is the spin operator and $U_{\mathrm{SO}}$ denotes an average spin-flip potential the gradient of which is taken to be a constant into all directions

$$
\operatorname{grad} U_{\mathrm{SO}}=\left(\sigma_{U}, \sigma_{U}, \sigma_{U}\right) .
$$

Depending on the kinematic properties of the electron's state the inverse of $\sigma_{U}$ gives rise to a distance, the spin-orbit scattering length $l_{S O}$, after which the electrons spin relaxes on average.

So far, we have considered a Hamiltonian modeling in a continuous real space representation. The randomness is characterized completely by a diagonal potential, Eqs. (33),34, 355). It is instructive to consider the same model in a discrete matrix representation and to ask for the resulting statistical properties. In a finite cubic system (with volume $L^{d}$ ) with periodic boundary conditions we can use the momentum representation, $\mathbf{p}|\mathbf{k}\rangle=\hbar \mathbf{k}|\mathbf{k}\rangle$, which diagonalizes the kinetic energy in Eq. (32) $(\mathbf{B}=0)$

$$
H_{\mathbf{k k}^{\prime}}=\frac{(\hbar \mathbf{k})^{2}}{2 m} \delta_{\mathbf{k k}^{\prime}}+\tilde{V}\left(\mathbf{k}-\mathbf{k}^{\prime}\right)
$$

where $\tilde{V}(\mathbf{k})$ is the Fourier transform of $V(\mathbf{r})$. The Gaussian white noise results in the correlation

$$
\left\langle\tilde{V}\left(\mathbf{k}_{1}-\mathbf{k}_{2}\right) \tilde{V}\left(\mathbf{k}_{3}-\mathbf{k}_{4}\right)\right\rangle=L^{-d} \gamma^{2} \delta_{\mathbf{k}_{1}-\mathbf{k}_{2}, \mathbf{k}_{3}-\mathbf{k}_{4}} .
$$


Thus, the random matrix $H_{\mathbf{k k}^{\prime}}$ has a diagonal deterministic part and a random part the average value of which vanishes, but with strong statistical correlations among those elements which fulfill momentum conservation. What can be seen here is that the statistical properties of a representing matrix for the Hamiltonian do, in general, depend on the chosen basis in Hilbert space.

The matrix $H_{\mathbf{k k}^{\prime}}$ can be taken to be finite dimensional if one restricts the wave lengths to be larger than a convenient microscopic scale, $a$. Then the matrix dimension is $\sim(L / a)^{d}$ and the system can be studied on a computer.

A direct modeling of disordered mesoscopic systems by a finite dimensional Hamiltonian matrix is given by a tight-binding version of the Gaussian white-noise potential and was introduced in the work of Anderson [8]. This Anderson model is defined on a cubic lattice with lattice constant $a$

$$
H=\sum_{\mathbf{m}} \varepsilon_{\mathbf{m}}|\mathbf{m}\rangle\left\langle\mathbf{m}\left|+\sum_{\left\langle\mathbf{m}, \mathbf{m}^{\prime}\right\rangle} t_{\mathbf{m}, \mathbf{m}^{\prime}}\right| \mathbf{m}\right\rangle\left\langle\mathbf{m}^{\prime}\right|,
$$

where $|\mathbf{m}\rangle$ denote tight-binding states situated at lattice point $\mathbf{m}$ and $\left\langle\mathbf{m}, \mathbf{m}^{\prime}\right\rangle$ means nearest neighbors only. The site energies $\varepsilon_{\mathbf{m}}$ correspond to the (random) potential energy and the (deterministic) hopping matrix elements $t_{\mathbf{m}, \mathbf{m}^{\prime}}$ to the kinetic energy. Again, the matrix dimension is $\sim(L / a)^{d}$ for a cubic system of linear size $L$. However, the matrix is sparse since the nearest neighbor condition leaves most elements vanishing.

In the absence of magnetic field the hopping strength is taken to be equal for all nearest neighbors (with coordination number $Z$ ) and set to 1 . This defines the kinetic energy band to be of width $2 Z$.

A homogeneous magnetic field $\mathbf{B}=(0,0, B)$ can be included in the kinetic energy by the Peierls substitution $[106$. In the Landau gauge, $\mathbf{A}=(0,-B x, 0)$, it reads in $2 \mathrm{D}$

$$
t_{\mathbf{m}, \mathbf{m}^{\prime}}(B)=t_{\mathbf{m}, \mathbf{m}^{\prime}}(0)\left\{e^{ \pm 2 \pi i \alpha m_{x}} \delta_{m_{x}^{\prime}, m_{x}} \delta_{m_{y}^{\prime}, m_{y} \pm 1}\right\} .
$$

Here $0<\alpha<1$ is the number of flux quanta $h / e$ per unit cell. In contrast to continuum models a feature of commensurability appears. Requiring commensurability between the lattice constant and the magnetic length restricts $\alpha$ to take rational values only

$$
\alpha=p / q .
$$

Furthermore, in this model the magnetic length is restricted to be larger than the lattice constant since the effect of magnetic field is assumed to be weak on the size of a unit lattice cell, i.e. only its influence on phases is taken into account by the Peierls substitution.

The statistical properties of the model are fixed once a distribution of the independent diagonal energies is chosen. A convenient choice is a box distribution on the interval $\left[-V_{0}, V_{0}\right]$ with correlation

$$
\left\langle\varepsilon_{\mathbf{m}} \varepsilon_{\mathbf{m}^{\prime}}\right\rangle=\frac{V_{0}^{2}}{3} \delta_{\mathbf{m m}^{\prime}}
$$


The parameter $V_{0}^{2} / 3$ can be identified with $\gamma^{2} /(\sqrt{\pi} a)^{d}(\gamma$ taken from the white-noise model of Eq. (35)) and determines the mean free path $l_{e}$ (see Eq. (59)). We mention that the inclusion of spin-orbit scattering into the Anderson model is also possible 48,10,51, and gives rise to a spin-orbit scattering length $l_{S O}$.

Equations (32,41) correspond to the most prominent models of disordered mesoscopic electron systems. In the following we want to discuss, how one can use these models to calculate some of the basic physical quantities.

\section{B. Physical Quantities by Green's Function}

As outlined in the appendix, for non-interacting Fermion models we can concentrate on zero temperature (see Eq. (A27)) and only need the resolvent of the Hamiltonian

$$
G^{ \pm}(E)=\left(E^{ \pm}-H\right)^{-1}, \quad E^{ \pm}=E \pm i \epsilon, \quad \epsilon \rightarrow+0
$$

to calculate the basic physical quantities (Eqs. (A24 - A32)). Choosing a convenient basis

one has to perform traces involving $G^{ \pm}(E)$. The knowledge of $G^{ \pm}(E)$ is equivalent to finding the eigenvalues $\varepsilon_{\alpha}$ and eigenvectors $\left|\psi_{\alpha}\right\rangle$ of $H$ since

$$
G^{ \pm}(E)=\sum_{\alpha} \frac{\left|\psi_{\alpha}\right\rangle\left\langle\psi_{\alpha}\right|}{E^{ \pm}-\varepsilon_{\alpha}}
$$

The dynamic content of the resolvent is expressed by its relation to the time evolution operator $U(t)=e^{-i H t / \hbar}$

$$
i \hbar G^{ \pm}(E)=\int_{0}^{ \pm \infty} d t e^{(i E \mp \epsilon) t / \hbar} U(t) .
$$

With this it can be concluded that the long time average of the time dependent probability, $P\left(\mathbf{r}, \mathbf{r}^{\prime} ; t\right)$, to find an electron at point $\mathbf{r}^{\prime}$ that was created at point $\mathbf{r}$ is related to the Green's function, $G^{ \pm}\left(\mathbf{r}, \mathbf{r}^{\prime} ; E\right)=\left\langle\mathbf{r}^{\prime}\left|G^{ \pm}(E)\right| \mathbf{r}\right\rangle$, by

$$
\overline{P\left(\mathbf{r}, \mathbf{r}^{\prime} ; t\right)}=\lim _{\epsilon \rightarrow+0} \frac{\epsilon}{2 \pi} \int d E\left|G^{+}\left(\mathbf{r}, \mathbf{r}^{\prime} ; E\right)\right|^{2} .
$$

That leads to the important interpretation that $(\epsilon / 2 \pi)\left|G^{+}\left(\mathbf{r}, \mathbf{r}^{\prime} ; E\right)\right|^{2}$ defines the long time transition probability from $\mathbf{r}$ to $\mathbf{r}^{\prime}$ for a given energy $E$.

The local density of states (LDOS) at $T=0, \rho\left(\mathbf{r} ; E_{\mathrm{F}}\right)=\left\langle\mathbf{r}\left|\delta\left(E_{\mathrm{F}}-H\right)\right| \mathbf{r}\right\rangle$ can be calculated by (see Eq. (

$$
\rho\left(\mathbf{r} ; E_{\mathrm{F}}\right)=-\pi^{-1} \operatorname{Im} G^{+}\left(\mathbf{r}, \mathbf{r} ; E_{\mathrm{F}}\right)=\frac{\epsilon}{\pi} \int d^{d} r^{\prime}\left|G^{+}\left(\mathbf{r}, \mathbf{r}^{\prime} ; E_{\mathrm{F}}\right)\right|^{2}=\sum_{\alpha}\left|\psi_{\alpha}(\mathbf{r})\right|^{2} \delta\left(E_{\mathrm{F}}-\varepsilon_{\alpha}\right)
$$


and the global (averaged over volume $L^{d}$ ) density of states (DOS) by

$$
\rho\left(E_{\mathrm{F}}\right)=L^{-d} \sum_{\alpha} \delta\left(E_{\mathrm{F}}-\varepsilon_{\alpha}\right)
$$

For a finite closed system the DOS consists of isolated $\delta$ peaks which have to be smeared out by attaching a finite width of the scale of the level spacing to the peaks or by averaging over the ensemble of disorder realizations. The average level spacing $\Delta$ at $E_{\mathrm{F}}$ can thus be obtained by

$$
\Delta=-\pi\left\langle\operatorname{Im} \operatorname{Tr} G^{+}\right\rangle^{-1}
$$

Furthermore, it is of conceptual importance to mention that the Green's function $G\left(\mathbf{r}, \mathbf{r}^{\prime} ; E\right)$ can be represented by a path integral, since

$$
\left\langle\mathbf{r}^{\prime}|U(t)| \mathbf{r}\right\rangle=\int d[\mathbf{r}(t)] \exp (i / \hbar) S[\mathbf{r}(t)]
$$

where the integration runs over all paths $\mathbf{r}(t)$ connecting initial $(\mathbf{r})$ and final $\left(\mathbf{r}^{\prime}\right)$ points and $S[\mathbf{r}(t)]$ denotes the classical action associated to the Hamiltonian of the electron. The stationary path of $S[\mathbf{r}(t)]$ yields the solution of the classical equation of motion.

As a first instructive example we calculate the ensemble average of the Green's function in the continuous model without magnetic field, using the momentum representation, Eqs. (39,40). Since we cannot solve the Schrödinger equation for arbitrary disorder potential we rely on a perturbative analysis,

$$
G^{+}=G^{+0} \sum_{n=0}^{\infty}\left(V G^{+^{0}}\right)^{n},
$$

where $\left\langle\mathbf{k}^{\prime}\left|G^{ \pm 0}(E)\right| \mathbf{k}\right\rangle=\left(E^{ \pm}-(\hbar \mathbf{k})^{2} / 2 m\right)^{-1} \delta_{\mathbf{k} \mathbf{k}^{\prime}}$ is the Green's function of the kinetic energy term. Due to the property Eq. (40) the ensemble averaged Green's function is diagonal in momentum representation, too. It can be written in the form

$$
\left\langle\mathbf{k}\left|G^{ \pm}(E)\right| \mathbf{k}\right\rangle=\left(E^{ \pm}-(\hbar \mathbf{k})^{2} / 2 m-\Sigma^{ \pm}(\mathbf{k})\right)^{-1}
$$

where $\Sigma^{ \pm}(\mathbf{k})$ is called the self-energy the imaginary part of which defines an inverse time scale which can be interpreted as an elastic mean free time $\tau$

$$
\frac{\hbar}{2 \tau}=\operatorname{Im} \Sigma^{+}(\mathbf{k})
$$

We mention that, in general, this $\tau$ must not be identical to the average momentum relaxation time due to random collisions.

The self-energy can now be calculated order by order in perturbation theory (for details cf. [2]). For weak scattering, i.e. for $l_{e} \gg \lambda_{F}$, it turns out to be sufficient to take the first non-vanishing contribution which yields 


$$
\Sigma^{+}(\mathbf{k})=L^{-d} \gamma^{2} \sum_{\mathbf{k}}\left\langle\mathbf{k}\left|G^{+0}\right| \mathbf{k}\right\rangle
$$

and improve it by replacing $G^{+0}$ with the full resolvent $G^{+}$in Eq. (56) making the approximation for the self energy self-consistent. It leads to

$$
\tau=\frac{\hbar}{2 \pi \gamma^{2} \nu}
$$

where $\nu$ is the average DOS which, for Fermi energies far off the kinetic energy band edges, is not changed as compared to the DOS without disorder, i.e.

$$
\nu\left(E_{\mathrm{F}}\right)=\frac{m S_{d}}{(2 \pi)^{d} \hbar^{2}}\left(\frac{2 \pi}{\lambda_{F}}\right)^{d-2}
$$

Consequently, the elastic mean free path is related to $\gamma$ and $\lambda_{F}$ by

$$
l_{e}=v_{F} \tau=\frac{h^{2} \hbar^{2} \lambda_{F}^{d-3}}{m^{2} \gamma^{2} S_{d}} \propto \frac{E_{\mathrm{F}}^{2} \lambda_{F}^{d}}{\gamma^{2}} \lambda_{F}
$$

Calculating the disorder average of the conductivity requires already the average over a product of two Green's functions (called two-particle Green's function) which is already a non-trivial task. Starting from the Kubo formula, Eq. (A28), for the velocity correlator, using the momentum representation and replacing the matrix elements of the resolvent by their average values leads back to the Drude formula with the momentum relaxation time given by Eq. (57). However, this procedure ignores completely that the average of two Green's functions does not decouple into a product of averages. In the context of the path integral representation for the Green's functions, Eq. (52), it becomes obvious that this method cannot cope with quantum interference effects. Thus, the mean free time as given by Eq. (57) corresponds to a Drude like conductivity when quantum interference is suppressed.

The symmetry properties of the disorder ensemble yield a translational and rotational invariant two-particle Green's function

$$
\left\langle\left|G^{+}\left(\mathbf{r}, \mathbf{r}^{\prime}, E\right)\right|^{2}\right\rangle=: K\left(\left|\mathbf{r}-\mathbf{r}^{\prime}\right|, E\right)
$$

With this function the longitudinal conductivity, as given by Eq. (A32), is

$$
\sigma=\frac{2 e^{2} \epsilon^{2}}{h} \int d^{d} r x^{2} K\left(|\mathbf{r}|, E_{\mathrm{F}}\right) .
$$

The diffusion constant $D(E)$ is defined by the long time limit of the square displacement with respect to the probability distribution $\epsilon K(r, E)$ as

$$
\rho(E) D(E) t(\epsilon):=\epsilon \int d^{d} r x^{2} K(|\mathbf{r}|, E)
$$


where $t(\epsilon)=h / 2 \epsilon$ is a growing time scale as $\epsilon$ is sent to +0 . Thus, Eq. (61) demonstrates the validity of the Einstein relation.

Furthermore, it allows for a first definition of a localization length by means of the Green's function, i.e. if $\epsilon K(|\mathbf{r}|, E)$ stays finite for arbitrary finite $\mathbf{r}$, but falls off as

$$
\epsilon K(|\mathbf{r}|, E) \propto \exp \left(-2|\mathbf{r}| / \xi_{0}\right)
$$

the average conductance of a large volume $L^{d}$ will show the localization phenomenon introduced in Eq. (31) with $\xi_{0}$ defining a localization length. Note, that this length is defined with respect to the average of $\left|G^{+}\right|^{2}$ and must not be identical to just $1 / q$ of the exponential

fall off of averages of powers $\left|G^{+}\right|^{2 q}$. Nevertheless, the factor of 2 in Eq. (63) follows the convention to associate the localization length with the modulus of the Green's function.

\section{The Statistical Problem}

We are now in the position to formulate the statistical problem of calculating physical quantities in mesoscopic electron systems. The system is described by an ensemble of Hamiltonian matrices in a certain matrix representation. The relevant physical quantities can be obtained from the Green's function $G^{+}\left(\mathbf{r}, \mathbf{r}^{\prime} ; E\right)$. The average value will only determine the average DOS and the short distance mean free path, however not the global transport behavior. To account for the latter, the averaged two-particle Green's function is needed in order to see localization effects. Fluctuation effects in global transport cannot be obtained on the basis of averaged two-particle Green's functions; at least four-particle Green's functions have to be considered. We see that this situation calls for a general approach to the distribution functions of $G^{+}\left(\mathbf{r}, \mathbf{r}^{\prime} ; E\right)$. The field theoretic approaches to mesoscopic systems commonly known as non-linear sigma-models are constructed to fulfill this purpose [132,46]. We will not enter this subject because of its technical complexity, but will here focus on some general considerations.

Owing to Eq. (46) the statistics of $G^{+}\left(\mathbf{r}, \mathbf{r}^{\prime} ; E\right)$ is contained in the joint probability distribution of eigenvalues, $\varepsilon_{\alpha}$, and eigenvectors, $\psi_{\alpha}$,

$$
\mathcal{P}\left(\varepsilon_{\alpha_{1}}, \psi_{\alpha_{1}}, \varepsilon_{\alpha_{2}}, \psi_{\alpha_{2}}, \varepsilon_{\alpha_{3}}, \psi_{\alpha_{3}}, \ldots\right)
$$

Of course, it seems hopeless to find a general method to determine this function for any given ensemble of Hamiltonian matrices.

To get some feeling about the nature of the statistical problem we consider the Hamiltonian in a finite basis $\{|i\rangle\}_{i=1, \ldots, N}$ as hermitean $N \times N$ matrix $H_{i k}=\langle i|H| k\rangle$. The diagonalizing unitary matrix $U \in \mathcal{U}(N)$ with matrix elements $U_{k \alpha}$ fulfills

$$
\sum_{i k} U_{\beta i}^{\dagger} H_{i k} U_{k \alpha}=\varepsilon_{\alpha} \delta_{\alpha \beta}
$$


It is related to the amplitude of an eigenstate $\psi_{\alpha}$ in the $\{|k\rangle\}$ basis by

$$
\psi_{\alpha}(k):=\left\langle k \mid \psi_{\alpha}\right\rangle=U_{k \alpha}
$$

We can think of the randomness of $H$ as being controlled by a large number of independent parameters, e.g. strength and position of point scatterers in space, or the on-site energies in the Anderson model. Each realization of the Hamiltonian represents one point in this high dimensional parameter space. We can ask what happens for a certain stochastic process $H(s)$ in this parameter space where $s$ denotes a fictitious time and each element $H(s)$ is weighted by the corresponding probability. The original $H$ corresponds to some arbitrary point, say $H\left(s_{0}\right)$. We can increase $s$ in small steps $\delta s$ which leads to

$$
H(s+\delta s)=H(s)+\delta H(s, \delta s)
$$

The corresponding unitary matrix $U(s)$ behaves then as

$$
U(s+\delta s)=U(s) \tilde{U}(s, \delta s)
$$

where $\tilde{U}(s, \delta s)$ denotes the unitary matrix that diagonalizes the Hamiltonian $H(s+\delta s)$ with respect to the eigenvector basis of $H(s)$. Thus, $U(s)$ evolves in a multiplicative manner. One could think of treating $\tilde{U}(s, \delta s)$ by perturbation theory with respect to $\delta s$ to derive closed evolution equations for the probability distribution of $U_{k \alpha}(s)$ and the corresponding set of eigenvalues $\varepsilon_{\alpha}(s)$. This has, so far, not been undertaken. However, recently Chalker et al. 32 adopted a related picture to investigate the eigenvalue statistics separately by relying on simplifying approximations. Here, we only wanted to stress, that the problem of the statistics of eigenvectors can be viewed as the problem of a random multiplicative process for unitary matrices.

As to the problem of the statistics of eigenvectors one can easily imagine two extreme situations. In the first situation the probability distribution of $U$ is peaked at a single fixed matrix. This matrix singles out a certain basis of eigenstates. We choose this matrix as the unit matrix, such that the Hamiltonian is diagonal in the initial basis

$$
H_{i k}=\varepsilon_{i} \delta_{i k} .
$$

The corresponding eigenstates are localized at certain 'sites', i.e.

$$
\psi_{\alpha}(k)=\delta_{\alpha k}
$$

The second extreme situation corresponds to an isotropic distribution for the unitary matrix. By this we mean that the probability density, $\mathcal{P}(U)$, to find a certain unitary matrix $U$ within the volume element, $d[\mathcal{U}(N)]$, is equal for all elements $U \in \mathcal{U}(N)$. The volume element itself stays invariant under the action of group transformations (invariant measure) $d[\mathcal{U}(N)]$, i.e. it does not single out any particular element. The corresponding eigenstates are also isotropically distributed among all possible eigenstates, no basis is preferred.

Of course, both extreme situations are not generic ones and we have to see for which values of physical scales they may appear. 


\section{SCATTERING MATRIX MODELING}

For mesoscopic conductors with several probes the d.c. conductance coefficients $G_{k m}$ are determined by asymptotic current correlators, Eq. (A20), and hence for independent Fermions at $T=0$ by asymptotic Green's functions, Eq. (A31). In a geometry where probes are represented as infinite leads attached to the conductor the asymptotic Green's functions determine the matrix of transmission amplitudes, $t_{k m}^{\alpha \beta}$, to scatter from channel $\alpha$ in lead $k$ to channel $\beta$ in lead $m$ and the Büttiker formula relates them to the conductance, Eq. (A36),

$$
G_{k \neq m}(\omega=0, T=0)=\frac{e^{2}}{h} \operatorname{Tr}\left\{t_{k m} t_{k m}^{\dagger}\right\}\left(E_{\mathrm{F}}\right) .
$$

This formula tells that the dimensionless conductance, especially in a two-probe geometry, is the number of effective transmitting modes,

$$
g=\sum_{\alpha} T_{\alpha}, \quad T_{\alpha}=\sum_{\beta}\left|t_{\alpha \beta}\right|^{2}, \quad 0 \leq T_{\alpha} \leq 1
$$

and it is thus of similar conceptual importance as the Thouless formula, Eq. (20).

The transmission matrix can be calculated by asymptotic Green's functions. These have to be determined under the requirement of attaching the leads to the conductor. For this purpose it is advantageous to divide the Hilbert space into two parts by the projection operator $P$ onto the Hilbert space of the conductor and its counterpart $Q=1-P$ that projects onto the leads. Denoting projected operators $A$ by $P A P=: A_{P P}, P A Q=: A_{P Q}$, etc. the general algebraic solution of the problem to calculate the projected Green's function reads

$$
G_{P P}^{+}(E)=\left(E^{+}-\tilde{H}_{P P}(E)\right)^{-1}
$$

where the effective (in general energy dependent and non-hermitean), Hamiltonian $\tilde{H}_{P P}$ is defined via the couplings and the Green's function of the leads $\tilde{G}_{Q Q}^{+}(E):=\left(E^{+}-H_{Q Q}\right)^{-1}$

$$
\tilde{H}_{P P}(E)=H_{P P}+H_{P Q} \tilde{G}_{Q Q}^{+}(E) H_{Q P} .
$$

Since Eq. (73) is defined for a finite system and the infinite clean leads Green's function can be determined analytically for not too complicated geometries the method of effective Hamiltonian also helps in doing the calculations numerically. The Green's function evaluated at the surface between leads and conductor will then determine the conductance coefficients.

The Büttiker formula points out that the mesoscopic conductor has very much in common with optical wave guides where transmission probabilities between incoming and outgoing wave modes are the central physical quantity of interest. It is thus tempting to model mesoscopic conductors by scattering matrices in analogy to optical wave guides. 


\section{A. Quasi-One-Dimensional Conductor}

As a guiding example let us consider a box shaped system (see Fig. 12 with two semiinfinite leads attached serving as particle reservoirs (contacts)). The cross section $L_{t}^{d-1}$ is kept fixed while the the length $L$ is treated variable. Such systems are denoted as quasione-dimensional (quasi-1D) conductors. The leads are characterized by discrete branches of energy dispersion (see Fig. 13) due to the finite extension in transverse direction. For a given value of the Fermi energy there exist $N_{c}$ quantum states of incoming and outgoing waves (channels). As a rough estimate one may think of $N_{c}$ being the number of lattice points one can put on the cross section of the conductor with lattice spacing of half a Fermi wavelength, $N_{c} \approx\left(2 L_{t} / \lambda_{F}\right)^{d-1}$.

A direct consequence of the Büttiker formula is the integer quantization of dimensionless conductance in ideal ballistic conductors where no scattering occurs, or the mean free path $l_{e}$ is much larger than $L_{t}, L$. Then $T_{\alpha}=1$ and the conductance displays quantized plateaus as a function of the Fermi energy (or particle density). Jumps from one plateau to another occur whenever a new channel is occupied, i.e. the Fermi energy crosses a new branch of the leads energy dispersion. In contrast to classical physics the conductance of an ideal conductor is not infinite but bounded by the finite number of quantum channels. The classical behavior can only be recovered for vanishing Fermi wavelength or, equivalently, for infinite number of channels. The finite resistance of ideal ballistic conductors can be attributed to the contacts where the equilibration of the electrons take place. Therefore, one may think of a decomposition of the resistance into a contact resistance, $R_{c}=\left(e / h^{2}\right) 1 / N_{c}$, and an intrinsic resistance, $\hat{R}$, such that $R=R_{c}+\hat{R}$. This yields for the corresponding intrinsic conductance $\hat{g}=\sum_{\alpha} T_{\alpha} /\left(1-N_{c}^{-1} \sum_{\alpha} T_{\alpha}\right)$, a result that was obtained by Langreth and Abrahams 87 generalizing the one-channel version of Landauer's pioneering works 84,85. However, one should keep in mind that the notion of intrinsic conductance is a theoretical construction

and not a quantity to be measured in a two-probe experiment. Such experiment corresponds to the conductance as described by the Büttiker formula.

In general, the quasi-1D conductor can be described by a $2 N_{c} \times 2 N_{c}$ scattering matrix $S$

$$
S=\left(\begin{array}{ll}
r & t^{\prime} \\
t & r^{\prime}
\end{array}\right)
$$

connecting incoming and outgoing channels left and right of a scatterer, $t, r, t^{\prime}$ and $r^{\prime}$ being $N_{c} \times N_{c}$ matrices of transmission and reflection coefficients for scattering from left to right and vice versa, respectively (see Fig. 14). Here $t$ is precisely the matrix of transmission amplitudes that appear in the Büttiker formula. Due to probability-flux conservation $S$ is unitary,

$$
S^{\dagger}=S^{-1}
$$


To model a long quasi-1D system one can now add several conductors in series. However, the total $S$-matrix is not multiplicative. Instead of

$$
\left(\begin{array}{c}
O \\
O^{\prime}
\end{array}\right)=S\left(\begin{array}{c}
I \\
I^{\prime}
\end{array}\right)
$$

we are seeking a transfer matrix $M$ with the property $M_{1+2}=M_{2} M_{1}$, i.e.

$$
\left(\begin{array}{c}
O^{\prime} \\
I^{\prime}
\end{array}\right)=M\left(\begin{array}{l}
I \\
O
\end{array}\right)
$$

A straightforward calculation yields

$$
M=\left(\begin{array}{cc}
\left(t^{\dagger}\right)^{-1} & r^{\prime} t^{\prime-1} \\
-t^{\prime-1} r & t^{\prime-1}
\end{array}\right)
$$

Making use of probability-flux conservation

$$
M \Sigma_{z} M^{\dagger}=\Sigma_{z}, \quad \Sigma_{z}=\left(\begin{array}{cc}
1 & 0 \\
0 & -1
\end{array}\right)
$$

where 1 stands for the $N_{c} \times N_{c}$ unit matrix we can rewrite the dimensionless two-probe conductance as

$$
g=\operatorname{Tr} \frac{2}{M M^{\dagger}+\left(M M^{\dagger}\right)^{-1}+2} .
$$

A modeling of a mesoscopic quasi-1D conductor can now be based directly on the scattering matrix or, equivalently, on the transfer matrix. This can be done by fixing the statistical properties of the $S$-matrix corresponding to a small strip of length $\delta L$, denoted as strip $S$-matrix $S(\delta L)$ and compose the whole conductor by putting statistically independent strip $S$-matrices in series. The total $S$-matrix follows then from the multiplication of the corresponding strip transfer matrices $M(\delta L)$. The assumption of statistical independence is justified if the strip length $\delta L$ is larger than the microscopic potential correlation length $l_{V}$ introduced in Sect. IIIB. Furthermore, this modeling allows for a simple description of the mean free path corresponding to the strip $S$-matrix. As long as the corresponding reflection probabilities $\left|r_{\alpha \beta}\right|^{2}=: R_{\alpha \beta}$ are small compared to 1 the mean free path $l_{e}$ is large compared to $\delta L$ and can be defined as follows

$$
\frac{\delta L}{l_{e}}:=N_{c}^{-2} \sum_{\alpha \beta}\left\langle R_{\alpha \beta}(\delta L)\right\rangle .
$$


The $S$-matrix fulfills the requirement of unitarity. To model systems with specific symmetry properties one can impose further symmetry constraints on $S$. The most important case is that of time reversal symmetry. This symmetry can be broken by magnetic fields. To model systems with magnetic field one faces the problem that the magnetic length (see Eq. (36)) as a chiral length scale has to be incorporated into the model. It is not obvious how to do that in the scheme described here. However, weak magnetic fields only break time reversal symmetry (the magnetic length being larger than system size) and can be simply incorporated in the symmetry properties of $S$. For example, time reversal operates by interchanging incoming and outgoing channels and complex conjugation of amplitudes. In case of time inversion symmetry the $S$-matrix and transfer matrix $M$ fulfill

$$
S=S^{\mathrm{T}}, \quad M^{*}=\Sigma_{x} M \Sigma_{x}, \quad \Sigma_{x}=\left(\begin{array}{ll}
0 & 1 \\
1 & 0
\end{array}\right) .
$$

Internal degrees of freedom, such as spin, can also be incorporated by taken the corresponding time reversal transformation into account.

So far, the modeling rests on a microscopic length scale, the mean free path $l_{e}$, and symmetry properties. The statistical problem is thus defined by (i) fixing the distribution of the strip $S$-matrix $S(\delta L)$ and (ii) by applying the composition law for the corresponding transfer matrices

$$
M(L+\delta L)=M(\delta L) M(L) .
$$

This defines a stochastic multiplicative process where the time variable is the system length. This resembles Eq. (68). (iii) The statistical properties of the increment

$$
\delta M(L, \delta L)=M(L+\delta L)-M(L)
$$

are known by construction. Following the steps (i) - (iii) it is possible to construct a stochastic differential equation (Fokker-Planck equation) for the distribution function $\mathcal{P}(M ; L)$ (see e.g. [94). Still, a general solution of this equation is presently not available and we will not discuss it in detail. A related approach, based on simplifying assumptions, will be discussed in Sect. VIIIB.

The matrices $t t^{\dagger}$ and $M M^{\dagger}$ occurring in the conductance formulas, Eqs. (71, 81), are hermitean and, thus, can be diagonalized. The positive eigenvalues are denoted as $0 \leq \mathcal{T}_{i} \leq 1$ (for $t t^{\dagger}$ ) and as $0 \leq e^{\nu_{i}}<\infty$ (for $M M^{\dagger}$ ). The eigenvalues of $M M^{\dagger}$ appear in inverse pairs and we can restrict to those with $\nu_{i} \geq 0$. The conductance reads in the corresponding eigenvalue representation

$$
g=\sum_{i} \mathcal{T}_{i}=\sum_{i} \frac{2}{1+\cosh \nu_{i}}
$$


Here the eigenvalues $\mathcal{T}_{i}$ have to be distinguished from the total probability to be scattered from a channel $\alpha$ into forward direction, $T_{\alpha}$, that occurs in Eq. (72). A related polar parameterization (cf. [121]) of the transfer matrix $M$ in terms of radial coordinates $0 \leq \lambda_{i}<$ $\infty$ and four unitary $N_{c} \times N_{c}$ matrices $u^{i}$ is also used frequently

$$
M=\left(\begin{array}{cc}
u^{1} & 0 \\
0 & u^{3}
\end{array}\right)\left(\begin{array}{cc}
\sqrt{1+\lambda} & \sqrt{\lambda} \\
\sqrt{\lambda} & \sqrt{1+\lambda}
\end{array}\right)\left(\begin{array}{cc}
u^{2} & 0 \\
0 & u^{4}
\end{array}\right) .
$$

Here $\lambda=\operatorname{diag}\left(\lambda_{1}, \ldots, \lambda_{N_{c}}\right)$ and

$$
\mathcal{T}_{i}=\left(1+\lambda_{i}\right)^{-1}
$$

It worth noticing that in these $S$-matrix models the conductance itself appears as a linear statistics 3 of eigenvalues of a certain random matrix. However, one should keep in mind that the statistical properties of these eigenvalues are, in general, not independent of those of the associated eigenvectors.

\section{B. Localization}

Before we introduce generalizations of the $S$-matrix modeling we will show that the present one is well suited to discuss the problem of localization in quasi-1D conductors.

An instructive example is the $1 \mathrm{D}$ conductor for which $N_{c}=1$. The composition law Eq. (84) tells that the composition law for the transmission reads (see Fig. 15)

$$
t_{12}=\frac{t_{1} t_{2}}{1-r_{1}^{\prime} r_{2}}
$$

Exploiting unitarity of $S$ yields for the transmission probability $T=|t|^{2}=1-R, R=|r|^{2}=$ $\left|r^{\prime}\right|^{2}$

$$
T_{12}=\frac{T_{1} T_{2}}{1-2 \cos \phi \sqrt{R_{1} R_{2}}+R_{1} R_{2}}
$$

where $\phi$ is the sum of phases of $r_{1}^{\prime}$ and $r_{2}$. Based on Eq. (90) one can derive an evolution equation for the probability distribution of $T$ with increasing length $L$. We will postpone this to Sect. VIIC. Here we will take advantage of the fact that an average of $\ln T_{12}$ over a uniform distribution of phases $\phi$ yields the simple expression [9]

\footnotetext{
${ }^{3}$ By linear statistics one denotes quantities that are sums of a specific function $f$ of the random parameters, $\sum_{i} f\left(\lambda_{i}\right)$.
} 


$$
\left\langle\ln T_{12}\right\rangle_{\phi}=\ln T_{1}+\ln T_{2}
$$

Thus, the phase-average of the logarithm of the transmission turns out to be additive. Iterating this procedure tells that the logarithm of the transmission will be distributed in a Gaussian way according to the central limit theorem for independent additive random numbers. This motivates to call $T_{\mathrm{t}}:=\exp \langle\ln T\rangle$ the typical transmission, and to write down a quantum series composition law for conductance $g=T$ in $1 \mathrm{D}$ conductors

$$
g_{\mathrm{t}}\left(L_{1}+L_{2}\right)=g_{\mathrm{t}}\left(L_{1}\right) g_{\mathrm{t}}\left(L_{2}\right)
$$

This composition law is in striking contrast to the macroscopic composition law for incoherent conductors, $G^{-1}\left(L_{1}+L_{2}\right)=G^{-1}\left(L_{1}\right)+G^{-1}\left(L_{2}\right)$. Equation (92) immediately tells that the conductance will exponentially decrease with increasing system length $L$, which means localization. To determine the localization length $\xi$ we calculate

$$
\frac{d g_{\mathrm{t}}(L)}{d L}=g_{\mathrm{t}}(L) \frac{g_{\mathrm{t}}(\delta L)-1}{\delta L}, \delta L \rightarrow 0 .
$$

Thus, $g_{\mathrm{t}}(L)=g_{\mathrm{t}}\left(L_{0}\right) \exp \left(-2\left(L-L_{0}\right) / \xi\right)$ with $\xi / 2=\delta L /\left(1-T_{\mathrm{t}}(\delta L)\right)$, and by Eq. (82)

$$
\xi=2 l_{e} .
$$

This result demonstrates that in one-dimensional conductors all states are localized. Furthermore, the localization already occurs on the scale of the mean free path. This is a crucial mesoscopic effect as it is a consequence of the multiplicative quantum series composition, Eq. (92).

For arbitrary channel numbers $N_{c}$ the above 1D result does not apply. However, one can make an educated guess what might happen to the localization length, if localization occurs at all.

To this end, we notice that the conductance in $1 \mathrm{D}$ for length $L$ much smaller than $l_{e}$ is given by $g(L)=1-L / l_{e}$ in accordance with our definition of $l_{e}$. Since the result $g=1$ corresponds to ideal transmission of the wave guide an Ohmic series decomposition into contact resistance 1 and intrinsic resistance, $L / l_{e}$, is meaningful. Assuming that for $N_{c}>1$ and $L \ll \xi\left(N_{c}\right)$ Ohm's parallel composition law is applicable yields the classical conductance

$$
g_{c l}(L)=\frac{N_{c} l_{e}}{L}
$$

and the natural limit for this law to hold, $g(L \approx \xi) \approx 1$, yields an estimate of $\xi\left(N_{c}\right)$

$$
\xi\left(N_{c}\right) \approx N_{c} l_{e}
$$

We are now going to discuss rigid results that confirm the localization effect for arbitrary $N_{c}$. In Sect. VIIIB we will see that our estimate of the quasi-1D localization length is in accordance with known results. 
A crucial point in the 1D calculation was the multiplicative composition law for typical conductance. The transfer matrix obeys also a multiplicative composition law and the question arises what can be said about the statistical properties of a large product of random transfer matrices. Recall that the product of independently distributed positive random numbers $X(i)$,

$$
\mathcal{X}(N)=\prod_{i=1}^{N} X(i)=\exp \left[\sum_{i=1}^{N} \ln X(i)\right]
$$

gives rise to a central limit theorem for its logarithm: For large $N$, the distribution of $\ln \mathcal{X}(N)$ is well approximated by a Gaussian with mean value $N\langle\ln X\rangle$ and variance

$$
\sigma_{N}^{2}=N\left[\left\langle(\ln X)^{2}\right\rangle-\langle\ln X\rangle^{2}\right] .
$$

For the original variable $\mathcal{X}(N)$ the distribution is called log-normal distribution and reads

$$
\mathcal{P}(\mathcal{X}, N) d \mathcal{X}=\frac{1}{\sqrt{2 \pi} \sigma_{N}} \exp \left[-\frac{1}{2 \sigma_{N}^{2}}(\ln \mathcal{X}-N\langle\ln X\rangle)^{2}\right] d \ln \mathcal{X}
$$

Furthermore, one can conclude that for almost all realizations the geometric mean asymptotically coincides with the typical value

$$
\lim _{N \rightarrow \infty}(\mathcal{X}(N))^{1 / N}=\exp (\langle\ln X\rangle)=: X_{\mathrm{t}} .
$$

There exist some extensions of the law of large numbers to the case of products of independent random transfer matrices (see [37]). A version of the theorem of Oseledec [103] guarantees the existence (with probability 1) of eigenvalues of the diagonalizable limiting matrix 126, 127]

$$
\mathcal{M}:=\lim _{N \rightarrow \infty}\left(M^{\dagger}(N) M(N)\right)^{\frac{1}{2 N}}
$$

( $N=L / L_{0}, L_{0}$ some fixed reference length) of the form

$$
\mathcal{M}=\left(e^{\gamma_{N_{c}}}, \ldots, e^{\gamma_{1}}, e^{-\gamma_{1}}, \ldots, e^{-\gamma_{N_{c}}}\right) .
$$

Motivated by the analogy between transfer matrices and time evolution matrices considered in chaotic dynamics the quantities $\gamma_{1}<\ldots<\gamma_{N_{c}}$ are called Lyapunov exponents.

The Lyapunov exponents $\gamma_{i}$ are asymptotic non-random numbers and are the socalled self averaging limit of the random numbers $\nu_{i}(N)$, the exponents of eigenvalues of $M^{\dagger}(N) M(N)$, divided by $2 N$

$$
\gamma_{i}=\lim _{N \rightarrow \infty} \frac{\nu_{i}(N)}{2 N}
$$

A system with channel number $N_{c}$ has thus $N_{c}$ characteristic length scales 


$$
\xi_{i}=\frac{L_{0}}{\gamma_{i}}=\lim _{L \rightarrow \infty} \frac{2 L}{\nu_{i}(L)}
$$

According to the conductance formula Eq. (86) the inverse of the smallest Lyapunov exponent yields the relevant localization length

$$
\xi=\frac{L_{0}}{\gamma_{1}}
$$

A theorem by Fürstenberg [57] states that the largest Lyapunov exponent is always positive. This proves localization in strictly 1D, but is meaningless for $N_{c}>1$. Virster [127] has later proofed that $\gamma_{1}$ is strictly positive, as long as $N_{c}$ is finite. Thus, in the quasi-1D limit $L \rightarrow \infty$ the localization length $\xi$ is finite.

We postpone the discussion of the statistics of $\nu_{i}(L)$ to Sect. VIIIB and proceed by introducing more general $S$-matrix models.

\section{Networks Of Scatterers}

So far, the modeling of mesoscopic systems by scattering matrices was restricted to the quasi-1D case. More general $d$-dimensional mesoscopic systems can be described by a model introduced by Shapiro [114,44]. Instead of taking a strip $S$-matrix one can consider a regular network (lattice spacing $a$ ) consisting of $N=$ volume $/ a^{d}$ sites and $N_{b}$ bonds (see Figs. 16, 17). Each site represents a scatterer and is described by a unitary $2 d \times 2 d$ scattering matrix $S$. The bonds represent free propagation between the blocks. Each bond carries two waves propagating in opposite directions. Each $S$-matrix transforms $2 d$ incoming amplitudes into $2 d$ outgoing amplitudes. To each bond amplitude a random phase is added which simulates an irregular lattice of scatterers which are distributed between distance $a$ and $a+\lambda_{F}$ where $\lambda_{F}$ is the wavelength of scattered waves.

By specifying appropriate boundary conditions one can describe multi-probe conductors within this network model. Consequently, physical quantities like conductance coefficients and even channel dependent transmission amplitudes can be studied with less effort than in systems described by a Hamiltonian.

Interesting is the fact that the $S$-matrix modeling does also allow to determine energy eigenstates and eigenvalues. This comes along with the following interpretation: mapping incoming to outgoing states by scattering matrices usually refers to a process consuming infinite time in order to guarantee energy conservation. However, if one allows for a certain uncertainty in the energy, a finite time is enough to follow the scattering process. Therefore, one can take the point of view that the scattering matrices at each site describe a unitary time evolution of states in one unit of time. By this interpretation the finite energy uncertainty is treated as negligible. Accordingly we define the unitary matrix of time evolution in a unit of time by [44,55, 74 


$$
\sum_{k} U_{i k} \psi(k ; t)=\psi(i ; t+1)
$$

Here $\{\psi(k ; t)\}$ forms, for each time $t$, a set of $2 N_{b}$ complex bond amplitudes. Normalizing them, $\sum_{i}^{2 N}|\psi(i)|^{2}=1$, makes them a proper choice for local wavefunctions. The unitary $2 N_{b} \times 2 N_{b}$ matrix $U$ is uniquely determined by the network of scattering matrices since it summarizes nothing but the scattering conditions at the sites. Energy eigenstates $\psi$ are stationary solutions of the unitary operator $U$

$$
U \psi=\psi
$$

and thus correspond to the eigenstates of $U$ with eigenvalue 1 . The energy of the underlying electron system enters here only parametrically through the scattering amplitudes. For simplicity, let us assume that the scattering strengths are equal at all nodes (abbreviated by one symbol $s$ ) and randomness enters only through the bond phases. Then $U$ will have an eigenstate to eigenvalue 1 only for a discrete set of scattering strengths, which correspond to a discrete set of energy eigenvalues of the underlying electron system. Instead of looking for the discrete set of energies one can also fix the energy and ask for the more general eigenvalue problem

$$
U(E) \psi_{\alpha}(E)=e^{i \phi_{\alpha}(E)} \psi_{\alpha}(E)
$$

$\psi_{\alpha}(E)$ are, for fixed $E$, a set of $2 N_{b}$ eigenstates at energy $E$ of slightly modified disorder configurations, each of the modifications being an overall shift in random phases on the links. The eigenphases $\phi_{\alpha}(E)$ can be considered as an energy excitation spectrum at energy $E$. Thus, the network models allow to investigate energy eigenvector statistics and, via the phases $\phi_{\alpha}(E)$, energy eigenvalue statistics.

The network models have a further advantage as compared to Hamiltonian models concerning numerical calculations. In case that only a finite resolution of energy scales is required one does not need to fully diagonalize the unitary operator. Instead, one iterates the time evolution step by step until a certain time scale is reached, the inverse of which yields the required energy resolution.

Finally, we like to point out that within the network models there are possibilities not only to include global symmetries but also characteristic length scales. For example by allowing for internal degrees of freedom (e.g. spin) the number of amplitudes on each bond can be increased and scattering strengths can be introduced that take the change of internal degrees of freedom into account. The inclusion of strong magnetic fields with their characteristic chiral character is also possible. No commensurability features like in the Anderson model with Peierls substitution do occur. Especially, one model of this category has become widely known as the Chalker-Coddington model [26] in 2D which only allows for scattering to the left and right (see Figs. 18, 19). 


\section{STATISTICS IN IDEALIZED SITUATIONS}

In this section we consider idealized mesoscopic systems and ask for the statistics of energy eigenvalues and eigenvectors. The idealization is due to the assumption that the statistical properties of both can be studied separately. We distinguish between the localized situation imitated by a diagonal Hamiltonian and the delocalized situation imitated by a standard random hermitean matrix having independent Gaussian distributed random entries.

We will review the energy level statistics in these systems and the statistics of the local box-probability $P\left(L_{b}\right)$ for an electron that is in an eigenstate $\psi(\mathbf{r})$ to be found in a certain box of volume $L_{b}^{d}$,

$$
P\left(L_{b}\right):=\int_{\text {box }} d^{d} r|\psi(\mathbf{r})|^{2}
$$

The complete information about the (energy) level statistics of a $N \times N$ Hamiltonian is contained in the joint probability density

$$
\mathcal{P}\left(\varepsilon_{1}, \ldots, \varepsilon_{N}\right)
$$

To simplify notations we will assume throughout that any reordering of levels in $\mathcal{P}$ does not change the probability. The simplest statistical quantity to be extracted is the average level density

$$
\nu(E):=\langle\rho(E)\rangle=N^{-1}\left\langle\sum_{\alpha} \delta\left(E-\varepsilon_{\alpha}\right)\right\rangle=\int d \varepsilon_{2} \ldots d \varepsilon_{N} \mathcal{P}\left(E, \varepsilon_{2}, \ldots, \varepsilon_{N}\right)
$$

defining the average level spacing $\Delta(E)=(N \nu(E))^{-1}$. The two-level correlation function is

$$
R(s)=\Delta^{2}\left\langle\sum_{\alpha} \delta\left(E-\varepsilon_{\alpha}\right) \sum_{\beta} \delta\left(E^{\prime}-\varepsilon_{\beta}\right)\right\rangle-1, \quad s:=\frac{E-E^{\prime}}{\Delta} .
$$

For simplicity we assumed that it is translational invariant within a band of states with $\Delta$ being independent of energy. By writing the energy separation in units of average level spacing $(s)$ one focuses on those properties that are independent of the chosen energy units. Quantities which contain information about $n$-level correlation functions are the $n$-level spacing distribution $Q(n, s)$ which yields the probability to find exactly $n$ levels in an energy interval of width $s$ (the center of which can be arbitrary). The probability to find exactly no level inside the interval defines the so-called level spacing distribution $P(s)=d^{2} Q(0, s) / d s^{2}$ which yields the probability density to find a level spacing $s$ with respect to $d s$. The average number $\langle n(s)\rangle$ of levels in an interval $s$ and its variance $\Sigma^{2}:=\left\langle n^{2}(s)\right\rangle-\langle n(s)\rangle^{2}$ are also of interest. The derivative of $\Sigma^{2}$ with respect to $\langle n\rangle$ defines (for large $\langle n\rangle$ ) a level compressibility

$$
\chi:=\lim _{\langle n\rangle \rightarrow \infty} \frac{d \Sigma^{2}}{d\langle n\rangle} .
$$




\section{A. Localized Phase}

We consider $N$ orthogonal energy states $\psi_{\alpha}, \alpha=1,2, \ldots, N$ each of which is localized to one of $N$ sites of a regular lattice (lattice spacing $a$ ) in an arbitrary dimension $d$, leading to a vanishing localization length. The corresponding energy eigenvalues are independent random variables taken from a distribution

$$
p(\varepsilon)=\frac{1}{\sqrt{2 \pi} \varepsilon_{0}} \exp \left(-\frac{\varepsilon^{2}}{2 \varepsilon_{0}^{2}}\right) .
$$

The corresponding random Hamiltonian is diagonal in site representation

$$
H_{i k}=\varepsilon_{i} \delta_{i k}
$$

with eigenstates

$$
\psi_{\alpha}(i)=\delta_{\alpha i} \text { for } \varepsilon_{\alpha}=\varepsilon_{i}
$$

This situation was already addressed in Sect. IVC as one of two extreme cases for the eigenvector statistics. As compared to the Anderson tight-binding Hamiltonian, Eq. (41), the Hamiltonian in Eq. (115) ignores the kinetic energy completely. The energy level statistics is contained in the joint probability distribution

$$
\mathcal{P}\left(\varepsilon_{1}, \ldots, \varepsilon_{N}\right)=\prod_{\alpha=1}^{N} p\left(\varepsilon_{\alpha}\right) .
$$

Consequently, the average level density is

$$
\nu(E)=p(E)
$$

and the average level spacing within the band $\left(|E| \ll \varepsilon_{0}\right)$ is $\Delta=1 /\left(N p_{0}\right), p_{0}:=\left(\sqrt{2 \pi} \varepsilon_{0}\right)^{-1}$. The two-level correlation function $R(s)$ reflects the fact that there is only auto-correlation between levels (up to $1 / N$ corrections).

$$
R(s)=\delta(s)-N^{-1}
$$

The level spacing distribution follows the Poisson law

$$
P(s)=\exp (-s)
$$

(that is why the ensemble is called Poisson ensemble) and the characteristic relation between number variance and average number of levels can be obtained

$$
\Sigma^{2}=\langle n\rangle
$$

indicating that the levels are compressible with compressibility 1. 
The probability to find an electron in a box of size $a$ in the eigenstate $\psi_{\alpha}$ is

$$
P_{\alpha}(a ; i)=\left|\psi_{\alpha}(i)\right|^{2}=\delta_{\alpha i}
$$

Increasing box sizes to $L_{b}>a$ does not change the result as long as the boxes are chosen to be non-overlapping and centered at the original point. Independently of the box size the probability is either zero, if the state is not located in the box or 1 in the opposite case.

For a given energy $E$ we consider the microcanonical average of $P_{\alpha}$

$$
P\left(E, L_{b} ; i\right):=\frac{\sum_{\alpha} \delta\left(E-\varepsilon_{\alpha}\right) P_{\alpha}\left(L_{b} ; i\right)}{\sum_{\beta} \delta\left(E-\varepsilon_{\beta}\right)}
$$

where a smearing-out of $\delta$-functions is understood. The smearing width $\Gamma(E)$ has to be adjusted such that isolated $\delta$-peaks are broadened, but not over many neighboring levels. A convenient choice is to take $\Gamma(E)$ of the order of the actual level spacing at $E$ in a given realization. The denominator in Eq. (123) is then $1 / \Gamma(E)$. Since the eigenvalues are independently distributed, the centers of the corresponding eigenstates are randomly distributed over the lattice points. Therefore, in almost any case one will find zero boxprobability at a certain site $i$, unless the energy $E$ coincides (within the scale of the smearing $\Gamma(E)$ ) with eigenvalue $\varepsilon_{i}$, in which case the box-probability is 1 . The average box-probability is finite and given by $\langle P(E)\rangle_{L_{b}}=(1 / N)\left(L_{b} / a\right)^{d}=\langle P\rangle_{L_{b}}$.

Thus, the probability distribution $\mathcal{P}\left(P ; L_{b}\right)$ of the box-probability is obtained as

$$
\mathcal{P}\left(P ; L_{b}\right) d P=\left[\delta(P)\left(1-\langle P\rangle_{L_{b}}\right)+\delta(P-1)\langle P\rangle_{L_{b}}\right] d P
$$

The average value is not characteristic for this distribution, since the main weight is given to $P=0$. However, e.g. the geometric mean

$$
P_{\mathrm{t}}\left(L_{b}\right):=\exp \langle\ln P\rangle_{L_{b}}=0
$$

reflects a typical box-probability. Alternatively, one may consider the median of the distribution (that value up to which half of the total weight is used up) which vanishes also and could be termed typical as well.

The LDOS $\rho(E, i)=\sum_{\alpha} \delta\left(E-\varepsilon_{\alpha}\right)\left|\psi_{\alpha}(i)\right|^{2}$ with a smearing-out of $\delta$-functions on the scale of $\Gamma(E)$ yields for the LDOS in a box, $\rho\left(E, L_{b} ; i\right)$

$$
\rho\left(E, L_{b} ; i\right):=\Gamma(E)^{-1} P\left(E, L_{b} ; i\right) .
$$

However, we must mention that this notion of LDOS in a box differs from that used by other authors (see e.g. [47]). If one considers the LDOS with a parametric Lorentzian smearing-out of $\delta$-functions,

$$
\rho(E ; \mathbf{r})=\sum_{\alpha} \frac{\Gamma}{2 \pi} \frac{1}{\left(E-\varepsilon_{\alpha}\right)^{2}+\Gamma^{2} / 4}\left|\psi_{\alpha}(\mathbf{r})\right|^{2},
$$


where $\Gamma / \Delta$ can vary from 0 to $\infty$ one can investigate parametrically the interplay of level and eigenvector statistics.

To simplify the discussion we will be often more restrictive and call

$$
\tilde{\rho}\left(E, L_{b} ; i\right):=\Delta^{-1} P\left(E, L_{b} ; i\right)
$$

the reduced LDOS in a box. This definition concentrates on the eigenvector statistics. Consequently, the distribution function reads

$$
\mathcal{P}\left(\tilde{\rho} ; L_{b}\right) d \tilde{\rho}=\left[\delta(\tilde{\rho})\left(1-\Delta\langle\tilde{\rho}\rangle_{L_{b}}\right)+\delta\left(\tilde{\rho}-\Delta^{-1}\right) \Delta\langle\tilde{\rho}\rangle_{L_{b}}\right] d \tilde{\rho} .
$$

In summary, ideal localized systems (with vanishing localization length $\xi$ ) are characterized by a compressible spectrum of uncorrelated energies and a LDOS in a box that typically vanishes, although the average DOS is finite.

\section{B. Delocalized Phase}

For the idealized delocalized phase we make the assumption that eigenstates are distributed isotropically within the space of all possible eigenstates for a $N \times N$ Hamiltonian matrix, $H_{i k}$. In other words the diagonalizing unitary matrices $U_{k \alpha}$, Eq. (65), are uniformly distributed with respect to the invariant measure $d[\mathcal{U}(N)]$. This situation was already addressed in Sect. IVQ as the second extreme case for the eigenvector statistics.

Therefore, we assume the distribution of the Hamiltonian matrix to be unitarily invariant. One of the simplest possible choices is the so-called Gaussian unitary ensemble (GUE) introduced by Wigner [134] when studying the level statistics of complex nuclei,

$$
\mathcal{P}\left(\left\{\operatorname{Re} H_{i k}, \operatorname{Im} H_{i k}\right\}\right) d[H]=\tilde{C}_{N} \exp \left(-\frac{N}{2 \mathcal{E}_{0}^{2}} \operatorname{Tr} H^{2}\right) d[H] .
$$

Here $\tilde{C}_{N}$ is a normalization constant, $\mathcal{E}_{0}$ some arbitrary energy scale and the volume element is defined in terms of the independent matrix elements of $H$ as

$$
d[H]=\prod_{1}^{N} d H_{i i} \prod_{i<k}^{N} d\left(\operatorname{Re} H_{i k}\right) d\left(\operatorname{Im} H_{i k}\right) .
$$

Since $\operatorname{Tr} H^{2}=2 \sum_{i<k}\left[\left(\operatorname{Re} H_{i k}\right)^{2}+\left(\operatorname{Im} H_{i k}\right)^{2}\right]+\sum_{i} H_{i i}^{2}$ the GUE describes a random matrix with all its elements uncorrelated. Each of the elements vanishes on average and its absolute value fluctuates, $\left\langle\left|H_{i k}\right|^{2}\right\rangle=\mathcal{E}_{0}^{2} / N$. If we think of the model in site-representation and compare it to the Anderson model we see that by Eq. (130) hopping terms are dominant and hops from one site to another are equally probable independent of the spatial distance. This means that we can associate a vanishing time scale $t_{D}$ to travel through the system. 
Introducing eigenvalues $\varepsilon_{\alpha}$ and eigenvectors $\psi_{\alpha}(k)=U_{k \alpha}$ one can transform the probability $\mathcal{P}\left(\left\{\operatorname{Re} H_{i k}, \operatorname{Im} H_{i k}\right\}\right) d[H]$ to these variables at the expense of introducing a Jacobian between the set of variables. Fortunately, the Jacobian can be calculated, the integration over $d[\mathcal{U}(N)]$ being a trivial normalization (since $\operatorname{Tr} H^{2}$ is unitarily invariant), and one obtains for the joint probability of eigenvalues

$$
\mathcal{P}\left(\varepsilon_{1}, \ldots, \varepsilon_{N}\right)=C_{N} \prod_{\alpha<\beta}\left(\varepsilon_{\alpha}-\varepsilon_{\beta}\right)^{2} \exp \left(-\sum_{\alpha}^{N} \frac{N}{2 \mathcal{E}_{0}^{2}} \varepsilon_{\alpha}^{2}\right) .
$$

The factors in front of the exponential are due to the Jacobian and ensure that the probability to find two levels close to each other vanishes; a phenomenon which is denoted as level repulsion. The factors can be rewritten as $\exp \left(2 \sum_{\alpha<\beta} \ln \left|\varepsilon_{\alpha}-\varepsilon_{\beta}\right|\right)$ such that the joint probability describes a classical Gibbs ensemble,

$$
\mathcal{P}\left(\left\{\varepsilon_{\alpha}\right\}\right)=C_{N} \exp \left[-\beta \mathcal{H}\left(\left\{\varepsilon_{\alpha}\right\}\right)\right]
$$

of a gas of particles with coordinates $\varepsilon_{\alpha}$ and a Hamiltonian

$$
\mathcal{H}\left(\left\{\varepsilon_{\alpha}\right\}\right)=\frac{1}{2} \sum_{\alpha \neq \beta} U\left(\varepsilon_{\alpha}, \varepsilon_{\beta}\right)+\sum_{\alpha} V\left(\varepsilon_{\alpha}\right)
$$

that contains a logarithmic two-body interaction $U(x, y)=-\ln |x-y|$ and a one-body (confining) potential $V(x)=N x^{2} /\left(2 \beta \mathcal{E}_{0}^{2}\right)$. The inverse temperature is $\beta=2$ for the GUE. A related ensemble of real symmetric matrices, reflecting time inversion symmetry, denoted as Gaussian orthogonal ensemble (GOE), gives rise to the same Gibbs ensemble, the only change being $\beta=1$ ( $\beta=4$ corresponds to spin systems with time reversal symmetry, for more details and review see [92]). The interpretation of the joint probability distribution of levels as a Gibbs-ensemble has led to an important mean-field approach to level statistics 433 which we will use later in the context of quasi-1D conductance (cf. Sect. VIIIB). In the standard random matrix of GUE and GOE a number of results are well known:

1. The average DOS is (for $N \gg 1$ ) given by Wigner's semi-circle law,

$$
\nu(E)=\frac{1}{\pi \mathcal{E}_{0}} \sqrt{1-\left(\frac{E}{2 \mathcal{E}_{0}}\right)^{2}}
$$

with average level spacing $(E \approx 0) \Delta=\pi \mathcal{E}_{0} / N$.

2. The two-level correlation function shows level-repulsion for $s \ll 1$ and decays $\sim-1 / s^{2}$ for $s \gg 1$. In the GUE it reads $(s \neq 0, N \rightarrow \infty)$

$$
R(s)=-\left(\frac{\sin \pi s}{\pi s}\right)^{2} \text {. }
$$


3. The level spacing distribution is very well approximated by Wigner's surmise

$$
P(s)=A_{\beta} s^{\beta} \exp \left(-B_{\beta} s^{2}\right)
$$

where e.g. $A_{2}=\pi / 2, B_{2}=4 / \pi$. The characteristic small $s$ behavior $\sim s^{\beta}$ reflects the power of level-repulsion characteristic of the symmetry of the Hamiltonian.

4. The level number variance $\Sigma^{2}$ depends only logarithmically on the average level number,

$$
\Sigma^{2} \propto \ln \langle n\rangle,
$$

which yields a vanishing level compressibility $\left(\sim\langle n\rangle^{-1} \rightarrow 0\right)$.

Eigenvectors $\psi_{\alpha}(k)=U_{k \alpha}$ are isotropically distributed uncorrelated Gaussian variables up to $1 / N$ corrections. More precisely, in the GUE the eigenvector components are complex with uncorrelated Gaussian distributed real and imaginary parts. The average of each is zero while the variance is, due to normalization, given as

$$
\left\langle\left(\operatorname{Re} \psi_{\alpha}(k)\right)^{2}\right\rangle=\left\langle\left(\operatorname{Im} \psi_{\alpha}(k)\right)^{2}\right\rangle=\frac{1}{2 N} .
$$

For the GOE the components are real with vanishing average and variance $\left\langle\left|\psi_{a}(k)\right|^{2}\right\rangle=N^{-1}$.

This fixes the distribution of the probability $P(E ; a)$ to find an electron in a box of size $a$ (a lattice spacing). In the GUE one finds the Rayleigh distribution

$$
\mathcal{P}(P ; a)=N \exp (-P N),
$$

and in the GOE the Porter-Thomas distribution

$$
\mathcal{P}(P ; a)=\left(\frac{N}{2 \pi P}\right)^{1 / 2} \exp (-P N / 2) .
$$

Both distributions follow immediately once an intensity of waves $\psi$ is considered which are large sums of either complex or real numbers. This leads, by the law of large numbers, to Gaussian distributions for $\psi$. Both distributions can be generalized to arbitrary box size $L_{b}$ by replacing $N^{-1}$ with $\langle P\rangle_{L_{b}}=N^{-1}\left(L_{b} / a\right)^{d}$. Characteristic of these distributions is the exponential tail and the fact that no parameter besides $\langle P\rangle_{L_{b}}$ occurs. Still, the average value is a typical value for the distribution; e.g. the median differs from the average value by only a factor of $\ln 2$.

The statistics for the reduced LDOS in a box, $\tilde{\rho}\left(E ; L_{b}\right)=\Delta^{-1} P\left(E ; L_{b}\right)$, is then straightforward.

In summary, ideal delocalized systems (with vanishing time scale $t_{D}$ ) are characterized by an incompressible spectrum of correlated energies and a LDOS in a box the distribution of which develops an exponential tail. Still, the average value is typical. 


\section{CONCEPT OF SCALING}

The level and eigenvector statistics of the idealized systems show a remarkable feature: No physical scale besides the kinematic scales, level spacing $\Delta$, total volume $N a^{d}$ and box volume $L_{b}^{d}$ enters the statistical quantities. The parameters of the models, $\varepsilon_{0}$ (for the localized phase), and $\mathcal{E}_{0}$ only define the energy unit for $\Delta$. No transport scale enters the models. This is consistent with the following interpretation: In the localized phase the only relevant scale is the localization length, which was treated as zero. In the idealized delocalized phase the only relevant scale is the time to cross the system, or equivalently the corresponding Thouless energy $E_{\mathrm{Th}}=h / t_{D}$ which was treated as infinite. We may thus expect that the statistics described in the foregoing section correspond to asymptotic situations for which the dimensionless conductance is either zero (localized phase) or infinite (delocalized) phase.

The question then arises how the statistical properties do change for finite conductance. Of particular importance are the statistical properties when approaching the LD transition. Which physical scales determine the statistical properties? A related question concerns the change of the statistical properties with increasing system size.

\section{A. Relevant Scales And $\beta$-Function}

Looking at the conductance formulas by Thouless and Büttiker, Eqs. (20,72), it is tempting to assume that the conductance itself is the (perhaps only) relevant quantity to distinguish localized and delocalized phases and to characterize the LD transition.

1. The localized phase with

$$
g \ll 1
$$

corresponds to only closed transport modes (channels) (Büttiker formula with $T_{\alpha} \ll 1$ ) and the existence of a finite localization length $\xi \ll L, g \sim \exp (-2 L / \xi)$.

2. The delocalized phase with

$$
g \gg 1
$$

corresponds to many open channels (Büttiker formula with $N_{c} \approx g$ modes of $T_{\alpha} \approx 1$ ) and a Thouless energy much larger than the level spacing, $E_{\mathrm{Th}} \gg \Delta$.

3. The LD transition is expected to occur when scales match, i.e.

$$
E_{\mathrm{Th}} \sim \mathcal{O}(\Delta), g \sim \mathcal{O}(1)
$$


According to the Büttiker formula only a number of $\mathcal{O}(1)$ modes are open and the localization length diverges. This divergence turns out to be algebraically on approaching the transition point,

$$
\xi \propto \tau^{-\nu}
$$

where $\tau$ measures the deviation of a tuning parameter (e.g. Fermi energy) from its critical value at the LD transition. The exponent $\nu$ is called critical exponent of the localization length.

By this reasoning a relevant length scale $\xi_{c}$ can be defined via the conductance: $\xi_{c}$ is that fictitious system size for which

$$
g\left(\xi_{c}\right) \sim \mathcal{O}(1) .
$$

In the localized phase $\xi_{c}$ can therefore be identified with the localization length, while in the delocalized phase $E_{\mathrm{Th}}\left(\xi_{c}\right) \sim \mathcal{O}\left(\Delta\left(\xi_{c}\right)\right)$.

As our considerations call for a description of the LD transition as a critical phenomenon we briefly review some aspects of the latter.

A thermodynamic system is described in terms of the coordinates of its equilibrium states manifold $\mathcal{M}$ (e.g. volume $V$, particle number $N$ and temperature $T$ ). Any thermodynamic quantity of interest can be calculated from a thermodynamic potential (e.g. the free energy $\mathcal{F}(V, T, N)$ ) which is an analytic function of these coordinates (e.g. the specific heat $c_{V}=$ $\left.\partial^{2} \mathcal{F} / \partial T^{2}\right)$. Generally, one can change the parameterization of $\mathcal{M}$ (e.g. volume $V \rightarrow$ pressure $p)$ to describe the same equilibrium state - at least locally. In the thermodynamic limit where $N$ and $V$ tend to infinity simultaneously leaving the density $n=N / V$ constant, it may happen that the thermodynamic potential is no longer an analytic function of its coordinates. In such a situation the state of the system is not uniquely described by only one set of coordinates manifesting in phases. When crossing the coexistence curve the thermodynamic potential jumps. Such a process is called a first-order phase transition. The jump is quantitatively described by the value of the latent heat. Assume that the coexistence curve ends in some point of the $(p, T)$ diagram which is called the critical point. Here the latent heat vanishes, the thermodynamic potential is continuous and the difference of densities between the phases vanishes. Yet, at the critical point, the thermodynamic potential is not analytic since its second derivatives are discontinuous. It is then helpful to look for a quantity which serves as an order parameter of the transition. It should be finite in one phase and vanishes in the other phase. A more restrictive requirement is that an order parameter should correspond to a local quantity $\varphi(\mathbf{r})$ the average value of which $m=\langle\varphi(\mathbf{r})\rangle$ vanishes with some power law at the critical point (e.g. the difference of the corresponding densities in the transition), 


$$
\begin{array}{ll}
m=0, & T<T_{c} \\
m \propto \tau^{\beta}, & T>T_{c} .
\end{array}
$$

The exponent $\beta$ is called the critical exponent of the order parameter and $\tau=\left|\left(T-T_{c}\right) / T_{c}\right|$. It is a necessary condition for such power law behavior that the phase transition is not of first order since a power law is the paradigm of a physical law with no scales involved. In a first order transition, however, the jump in the thermodynamic potential sets a physical energy scale. A defining characteristic of a critical phenomenon is that there is also no relevant length scale at the critical point. Thus, the correlation length $\xi_{c}$ of the order parameter, $m=\langle\varphi(\mathbf{r})\rangle$, defined by the statistical properties of the local order parameter field

$$
\chi(r):=\langle\varphi(0) \varphi(\mathbf{r})\rangle \propto \exp \left(-r / \xi_{c}\right), \quad r:=|\mathbf{r}|,
$$

has to diverge at $T_{c}$ with a power law with some critical exponent $\nu$,

$$
\xi_{c} \propto \tau^{-\nu}
$$

The crucial assumption in any scaling approach to critical phenomena is that the critical exponents $\beta, \nu$ have their origin in the divergence of a single relevant length scale, the correlation length $\xi_{c}$. At the critical point where $\xi_{c}$ diverges the correlation function obeys a power law

$$
\chi(r) \propto r^{-\tilde{\eta}}
$$

Using $\xi_{c}$ as a cutoff when calculating the global susceptibility $\chi=\int d^{d} r \chi(r)$ we find a scaling relation

$$
\tilde{\eta}=\frac{2 \beta}{\nu}
$$

Let us now return to finite systems of linear size $L$ and assume that both the distance $r$ and the finite system size $L$ are within the regime without length scales and that the function $C(r, L):=\langle\varphi(0) \varphi(\mathbf{r})\rangle_{L}$ obeys a power law with respect to both lengths

$$
C(r, L) \propto r^{-\eta} L^{-y}
$$

The new exponent $y$ describes the system length dependence and $\eta \neq \tilde{\eta}$ describes the distance dependence. To consider this case turns out to be relevant in the LD transition problem as will be discussed in more detail in Sect. IXA. A similar reasoning as above yields now

$$
y+\eta=\tilde{\eta}=\frac{2 \beta}{\nu}
$$

Notice that the quantity $\eta$ is not determined by $\beta$ and $\nu$ alone but requires the additional knowledge of the unusual exponent $y$. 
Far away from the critical regime there may still exist a scaling variable $\Lambda(L)$ which replaces the role of $T$ in the following sense. The state of the system is described for a finite size $L$ already by the quantity $\Lambda(L)$ and the flow with increasing system size $L$ is determined by a single function of this variable, the so called $\beta$-function

$$
\beta(\ln \Lambda):=\frac{d \ln \Lambda(L)}{d \ln L}
$$

which should be a function of $\ln \Lambda$ alone. Provided the $\beta$-function is smooth, the flow is determined by the solution of the differential equation,Eq. (154), frequently called a renormalization group equation. The regime where $\beta(\ln \Lambda)$ can be linearized around a fixed point $\Lambda^{*}$ of the flow $\left(\beta\left(\ln \Lambda^{*}\right)=0\right)$,

$$
\beta(\ln \Lambda)=\beta^{\prime}\left(\ln \Lambda-\ln \Lambda^{*}\right),
$$

with $\beta^{\prime}$ being the slope of the $\beta$-function at $\Lambda^{*}$, is called the critical regime. It is in this regime where power law scaling can be expected. If we start with a system of size $L=L_{0}$, $\Lambda=\Lambda_{0}$ chosen close to $\Lambda^{*}$, and turn on the renormalization flow until the system reaches a size $\xi_{c}$ determined by the range of validity of Eq. (155) (which is here by definition the correlation length) we get

$$
\left(\frac{\xi_{c}}{L_{0}}\right)^{\beta^{\prime}}=\left(\frac{\ln \Lambda-\ln \Lambda^{*}}{\ln \Lambda_{0}-\ln \Lambda^{*}}\right) .
$$

Assuming that the critical regime is narrow, we can expand $\Lambda\left(\xi_{c}\right)$ and $\Lambda_{0}$ around $\Lambda^{*}$ and get for the correlation length

$$
\xi_{c}=L_{0}\left(\frac{\Lambda-\Lambda^{*}}{\Lambda_{0}-\Lambda^{*}}\right)^{1 / \beta^{\prime}} .
$$

The width of the critical regime $\Delta \Lambda=\Lambda_{0}-\Lambda^{*}$ is determined by $\tau$ such that

$$
\xi_{c} \propto(\Delta \Lambda)^{-\nu} \propto \tau^{-\nu}
$$

Thus, the exponent $\nu$ is given by the inverse slope of the $\beta$-function at the fixed point

$$
\nu=1 / \beta^{\prime}\left(\Lambda^{*}\right)
$$

The behavior of the scaling variable close to the transition is therefore of the form

$$
\Lambda(L ; \tau)=\Lambda^{*}+A \tau L^{1 / \nu}+\mathcal{O}\left(\tau^{2}\right)
$$

The coefficient $A$ can be brought to the form $A \tau L^{1 / \nu}=\tilde{A}\left(L / \xi_{c}\right)^{1 / \nu}$ where $\tilde{A}$ is of order $\Lambda^{*}$. Note, that, in general, the coefficient $\tilde{A}$ can differ on both sides of the transition, while the critical exponent $\nu$ is the same. 
It is crucial that any other scaling variable $\tilde{\Lambda}$ which is a smooth function of the previous one leads to the same value of $\nu$, although the overall form of its $\beta$-function can differ. This reflects the fact that critical exponents are universal numbers characterizing a whole class of systems. Such systems may differ by microscopic details, however the critical behavior is characterized by universal critical exponents of a few relevant quantities.

In Sect. IVB we have learned that knowing the Green's function is, in principle, enough to describe the transport properties including the LD transition. A natural guess for the order parameter field is the LDOS since it typically vanishes in the localized phase and is finite in the delocalized phase. Consequently, the average DOS (which is determined by the one-particle Green's function) appears as the natural (formal) order parameter. Since the average DOS does not show the LD transition but is a smooth function of the energy (which, in the problem of the LD transition, is the analog of temperature in equilibrium phase transitions) the critical exponent $\beta$ of this formal order parameter vanishes

$$
\beta=0
$$

The LD transition is not manifested in the one-particle Green's function, but in transport related quantities such as the two-particle Green's function. We like to mention that this fact rules out the possibility of a straightforward mean-field theory for the LD transition.

Furthermore, as the scaling relation Eq. (151) tells us the correlation exponent of the local susceptibility (a two-particle Green's function) vanishes,

$$
\tilde{z}=0
$$

This result seems to rule out a power law behavior with finite power for the two particle Green's function (say the density correlator) at criticality. In spite of that a finite power has been observed [27] for the density correlator at a LD transition. However, it turned out that it corresponds to a regime where the correlation function shows a power law (with unusual exponent $y$ ) also with respect to the system size $L$.

A further unusual feature of the LD transition, which at first glance seems to be independent of the previous ones, was first pointed out by Al'tshuler et al. [5] when reexamining the phenomenological scaling theory of Abrahams et al. [1].

This theory picks up the ideas that we introduced in the beginning of this section. The conductance $g(L)$ of a cube with linear dimension $L$ is treated as a scaling variable, i.e. $g$ obeys a differential equation in terms of a $\beta$-function which, for a certain universality class, is a unique function of $\ln g$. The fact that one scaling variable is enough to describe the transition is referred to as one-parameter scaling theory.

That such treatment makes sense can be seen when calculating the $\beta$-function for extreme situations. In a good metal with a conductivity that is a material constant, Ohm's law, Eq. (17), yields

$$
\beta(\ln g) \equiv d-2
$$


while in the exponential localized phase one obtains

$$
\beta(\ln g)=\ln g+A_{d}
$$

where $A_{d}$ is a constant for each dimension $d$. Assuming $\beta(\ln g)$ to be a smooth function one may interpolate between the asymptotic branches (see Fig. 20). From this picture one concludes: In 1D all states are localized, in 3D a generic LD transition takes place and for $2 \mathrm{D}$ the situation depends on the sign of weak-localization effects for large $g$. If these tend to localize, the $\beta$-function stays negative and all states will localize in the large $L$ limit. For weak anti-localization (found in 2D systems with spin-orbit interaction 63, 18, a LD transition should occur. It has been observed by numerical model calculations (see e.g. [51]). In an experiment by Kravchenko et al. 80 on a strongly interacting 2D electron system a LD transition was observed. At present it is not clear if it can be due to strong spin-orbit scattering. The quantum Hall effect, as mentioned in the introduction, is exceptional in this picture. This is due to the absence of a metallic phase. The corresponding $\beta$-function terminates from below at the critical point.

Although the one-parameter scaling theory by Abrahams et al. [1] is simple, predictive and qualitatively correct, it has an obvious shortcoming as was pointed out by Al'tshuler et al. [5]. The conductance of a mesoscopic system strongly depends on the individual system properties (e.g. on the given disorder potential). As a result, an ensemble of different systems exhibits conductance fluctuations which can become so strong that the corresponding distribution cannot be characterized by the mean value alone. The latter as well as higher moments, can be drastically influenced by the far tails of the distribution. It can thus happen that the mean value of the conductance $\langle g\rangle$ can not serve as a scaling variable.

Instead, an appropriate scaling approach to the LD transition has to be set up in terms of the complete distribution function of the conductance. However, as pointed out by Shapiro 116] it may be possible to apply the renormalization group approach to certain parameters occurring in that distribution function. If an appropriate parameter of the distribution exists which is less influenced by the far tails and which can serve to define a typical conductance, $g_{\mathrm{t}}$, it may be possible to find a flow equation in terms of a $\beta$-function for this typical conductance. In this sense the theory by Abrahams et al. can still be complete. For this scenario to be realistic, at least in the vicinity of the transition point, only one length scale $\xi_{c}$ should diverge at criticality. Then the LD transition obeys one-parameter scaling and the exponent $\nu$ of the correlation length $\xi_{c}$ is given by the $\beta$-function of $g_{\mathrm{t}}$.

It is worth mentioning that the conductivity, defined by the conductance as $\sigma(L)=$ $G(L) L^{2-d}$, is often referred to as an order parameter of the transition. It vanishes at the transition point in $d>2$ and $\sigma(L \rightarrow \infty, \tau) \propto \tau^{s}$ defines an critical exponent $s$. However, this notion of conductivity gives no further information than that already contained in the conductance. Only for $d>2$ the conductivity does vanish at the transition point and the exponent $s$ is trivially related to $\nu$ by $s=(d-2) \nu$ since $G(\xi)-G^{*} \propto \tau \xi^{1 / \nu}$. This relation is 
sometimes referred to as a scaling relation between critical exponents $s$ and $\nu$ suggesting that this replaces the scaling relation Eq. (151) in common critical phenomena. To the author this interpretation is misleading. Firstly, the conductivity is defined via the conductance, which is the scaling variable of the problem. For any critical phenomenon with a scaling variable $\Lambda(L)$ one can define similar order parameters by simply multiplying the scaling variable by a negative power of $L$. It is obvious that this does not give further insight. Secondly, for LD transitions occurring in $d=2$ the conductivity stays finite at the transition, which invalidates the notion of an order parameter for $\sigma$. Thirdly, the conductivity is not related to a local field of the problem.

In Sect.[X] we will see that the choice of an appropriate order parameter and the occurrence of broad distributions of physical quantities are intimately interrelated.

\section{B. Scaling Of Typical Values}

In this section we will exploit our experience with the notion of typical conductance in 1D and make an extension to higher dimensions following a work by Shapiro [116]. The extension relies on uncontrolled approximations and may thus be only of pedagogical interest. However, the results are surprisingly far-reaching and consistent with commonly accepted results about LD transitions.

Recall that the typical transmission probability in 1D followed a simple multiplicative composition law which under a scale transformation $L \rightarrow b L$ leads to the scaling law in 1D

$$
T_{\mathrm{t}}(b L)=\left(T_{\mathrm{t}}(L)\right)^{b} .
$$

We would like to generalize this law to higher dimensions. The multiplicative nature of the composition law is a consequence of the multiplicative nature of transfer matrices when put in series. If the cross section of the conductor is finite the transfer matrix acquires more modes. The parallel composition law for classical conductance treats all modes equal and so do we. To this end, we first have to define an appropriate conductance for a quasi-1D system for which the parallel composition makes sense. The notion of intrinsic resistance $\hat{R}$ and conductance $\hat{g}=\hat{R}^{-1}$, respectively, is helpful. In 1D we have $\hat{R}=(1-T) / T$ which can take all positive values. By Eq. (165) the series composition law reads

$$
\hat{R}(b L)=(1+\hat{R}(L))^{b}-1 .
$$

We adopt this law for any series composition in quasi-1D when we put $b$ blocks of size $L^{d}$ in series. That this makes sense can be seen by considering the cases with $\hat{R}(L) \gg 1$ and $\hat{R}(L) \ll 1$, respectively. For weak resistivity of each block, the resistances behave additive like in classical resistor networks. However, for strong resistivity of each block we recover the multiplication law leading to strong localization which is consistent with the finding of 
strong localization in quasi-1D. Once we have put the $b$ blocks in series, forming a quasi-1d conductor of cross section $L^{d-1}$ and length $b L$ we put $b^{d-1}$ of such conductors in parallel, resulting in a cubic conductor of total volume $(b L)^{d}$. For this composition we assume the classical parallel composition law to be valid f,

$$
\hat{R}(b L)=b^{1-d}\left[(1+\hat{R}(L))^{b}-1\right] .
$$

The application of parallel composition to arbitrary scaling factors can become quite inadequate. However, we will now restrict to very small scaling factors $b=1+\zeta, \zeta=\delta L / L \ll 1$ since we are only interested in the change of $\hat{R}$ under a small increase of system size. By this we can easily derive the corresponding $\beta$-function for $\hat{R}$,

$$
\frac{d \ln \hat{R}}{d \ln L}=(1-d)+\frac{1+\hat{R}}{\hat{R}} \ln (1+\hat{R}) .
$$

For easier comparison with the scaling theory of Abrahams et al. we reformulate the result in terms of the intrinsic conductance

$$
\beta(\ln \hat{g})=\frac{d \ln \hat{g}}{d \ln L}=(d-1)-(1+\hat{g}) \ln \left(1+\hat{g}^{-1}\right) .
$$

One sees immediately that this $\beta$-function has the same asymptotic behavior as in the scaling theory by Abrahams et al., i.e. for $\hat{g} \gg 1, \beta(\ln \hat{g}) \approx d-2-\hat{g}^{-1} / 2$, and for $\hat{g} \ll 1$, $\beta(\ln \hat{g}) \approx \ln \hat{g}$. Furthermore, it has no zero for $d \leq 2$ (see Fig. 21) and for $d=2+\epsilon$ with $\epsilon \ll 1$ one recovers the results $\hat{g}^{*}=1 / \epsilon=\nu$ of a much more involved field theoretic calculation [133] which is expected to be exact in the limit $\epsilon \ll 1$. In addition, one finds for $d=3$ a value of $\nu \approx 1.65$ which is close to known results $(\nu \approx 1.4$ 62, 64, 65]) of numerical calculations for $3 \mathrm{D}$ models .

\section{Scaling Of Distribution Functions}

We are now going to address the flow of distribution functions. As a guiding example we consider the 1D conductor. Here localization on the scale of the mean free path $l_{e}$ dominates and we can expect to get an almost complete description.

The composition law for transmission, Eq. (90), translates to that of intrinsic resistance, $\hat{R}$,

$$
\hat{R}_{12}=2 \hat{R}_{1} \hat{R}_{2}+\hat{R}_{1}+\hat{R}_{2}-2 \cos \phi \sqrt{\hat{R}_{1} \hat{R}_{2}\left(1+\hat{R}_{1}\right)\left(1+\hat{R}_{2}\right)}
$$

\footnotetext{
${ }^{4}$ Note, that this procedure is not capable of neither strong magnetic field nor spin-orbit interaction. In both of these cases a quantum parallel composition has to be constructed.
} 
We can use this to derive an evolution equation for the distribution function $\mathcal{P}(\hat{R} ; L)$. Since the procedure is of general importance we describe the essential steps (cf. [112]): a stochastic quantity $X(t)$ depending on time $t$ may increase in time $\delta t$ by $\delta X(t)=X(t+\delta t)-X(t)$. If the stochastic process is Markovian, i.e. it is determined by transition probabilities from one time step to the next and is not influenced by the history of the process the essential information is contained in the moments, $\left\langle(\delta X(t))^{n}\right\rangle$. If, in addition, only the first two moments scale linear with $\delta t$ (while all higher moments scale with higher powers) a FokkerPlanck equation (FPE) results

$$
\frac{\partial \mathcal{P}(X ; t)}{\partial t}=-\frac{\partial\left[\mathcal{D}^{1}(X) \mathcal{P}(X ; t)\right]}{\partial X}+\frac{\partial^{2}\left[\mathcal{D}^{2}(X) \mathcal{P}(X ; t)\right]}{\partial X^{2}}
$$

where $\mathcal{D}^{1}(X)=\langle\delta X(t)\rangle / \delta t$ and $\mathcal{D}^{2}(X)=\left\langle(\delta X(t))^{2}\right\rangle / 2 \delta t$ (for $\delta t \rightarrow 0$ ) are called drift and diffusion function, respectively.

In our problem, the stochastic quantity is $\hat{R}(L)$ and by Eq. (170) we obtain

$$
\delta \hat{R}(L)=2 \hat{R}(L) \hat{R}(\delta L)+\hat{R}(\delta L)-2 \cos \phi \sqrt{\hat{R}(L) \hat{R}(\delta L)(1+\hat{R}(L))(1+\hat{R}(\delta L))} .
$$

The average is to be taken over random phases $\phi$ and over the strip-resistance $\hat{R}(\delta L)$ which introduces the mean free path $\langle\hat{R}(\delta L)\rangle=\delta L / l_{e}$. This definition is often referred to as local weak scattering condition and is the basis of a FPE approach to the statistics. Equation (172) shows that only the first and second moments are linear in $\delta L$ while higher moments scale with higher powers. Thus, a FPE can be derived for $\mathcal{P}(\hat{R}, L)$ with drift and diffusion given by

$$
\mathcal{D}^{1}=\frac{2 \hat{R}+1}{l_{e}}, \quad \mathcal{D}^{2}=\frac{\hat{R}^{2}+\hat{R}}{l_{e}} .
$$

The FPE can be brought to the form [95]

$$
\frac{\partial \mathcal{P}(\hat{R} ; L)}{\partial L}=\frac{1}{l_{e}} \frac{\partial}{\partial \hat{R}}\left[\left(\hat{R}^{2}+\hat{R}\right) \frac{\partial \mathcal{P}(\hat{R} ; L)}{\partial \hat{R}}\right] .
$$

This distribution depends on only one parameter, the mean free path $l_{e}$. Two limiting cases can be considered now: (i) $\hat{R} \ll 1$ and (ii) $\hat{R} \gg 1$.

For case (i) the solution is an exponential distribution

$$
\mathcal{P}(\hat{R} ; L)=\frac{l_{e}}{L} \exp \left(-\hat{R} l_{e} / L\right)
$$

with average value $\langle\hat{R}(L)\rangle=L / l_{e}$. This solution corresponds to system sizes $L \ll l_{e}$.

For case (ii) the solution is a log-normal distribution

$$
\mathcal{P}(\hat{R} ; L) d \hat{R}=\frac{1}{\sqrt{4 \pi L / l_{e}}} \exp \left[-\frac{\left(\ln \hat{R}-L / l_{e}\right)^{2}}{2\left(2 L / l_{e}\right)}\right] d \ln \hat{R}
$$


with a typical value $\hat{R}_{\mathrm{t}}=\exp \langle\ln \hat{R}\rangle=\exp \left(L / l_{e}\right)$. This solution corresponds to localization with $L \gg l_{e}$, and a typical localization length of $\xi=2 l_{e}$, consistent with our previous finding (see Eq. (94)). Interesting is the fact that the second parameter of the log-normal distribution, the $\log$-variance $2 L / l_{e}$, is simply related to the log-average value

$$
\left\langle(\ln \hat{R}-\langle\ln \hat{R}\rangle)^{2}\right\rangle=2\langle\ln \hat{R}\rangle=2 L / l_{e}
$$

The log-normal form of the distribution for $L \gg \xi$ is also consistent with the general statement, Eq. (103), that the localization length $\left(\frac{2 L}{\nu_{1}}\right)$ corresponds to normally distributed quantities that become self averaging in the limit $L \rightarrow \infty$.

The distribution $\mathcal{P}(\hat{R} ; L)$ is broad even for $L \ll \xi$ (since an exponential tail already introduces a strong growth of moments $\propto n !)$ and causes large fluctuations for $\hat{R}$. The observation of broad distributions is central to mesoscopic systems and therefore we make some general statements about them. Following Shapiro [116] we formulate the concept of one-parameter scaling for broad distributions as follows.

A distribution of a physical quantity $X$ that depends on system size $L$ and a set of initial parameters $\left\{\alpha_{n}\right\}, \mathcal{P}\left(X ; L ;\left\{\alpha_{n}\right\}\right)$, obeys one-parameter scaling if (for large $L$ ) it is approximately a function of only $X$ and one scale dependent parameter $\alpha_{L}$,

$$
\mathcal{P}\left(X ; L ;\left\{\alpha_{n}\right\}\right) \approx F\left(X ; \alpha_{L}\right)
$$

and the flow of the parameter $\alpha_{L}$ is determined by a $\beta$-function

$$
\beta\left(\ln \alpha_{L}\right)=\frac{d \ln \alpha_{L}}{d \ln L} .
$$

That means that the whole set of initial parameters $\left\{\alpha_{n}\right\}$, needed to specify the initial smallscale distribution, disappear into the single parameter $\alpha_{L}\left(\left\{\alpha_{n}\right\}\right)$. The exact distribution does contain all the information about the initial set of parameters; however, the information can be accumulated in the very far tails of the distribution. By very far tail of a distribution we denote that range of values $X<X_{1}, X>X_{2}$ that is not statistically significant to some small but fixed probability $\delta$

$$
\int_{X_{1}}^{X_{2}} d X \mathcal{P}(X ; L)=1-\delta .
$$

By Eq. (178) it is meant that the approximation for the main part of the distribution is exact up to corrections of order $\delta$. For sufficiently large $L$ it can be taken to be arbitrarily small. Still, the moments of the distribution might be dominated by the very far tails of the exact distribution. Therefore, one cannot expect that in general the parameter $\alpha_{L}$ is simply related to moments of the distribution.

As a consequence of one-parameter scaling there should exist a universal limiting distribution at a critical point 


$$
\lim _{L \rightarrow \infty} \mathcal{P}(X ; L)=\mathcal{P}^{*}(X)
$$

which is fixed by the critical value $\alpha^{*}=\lim _{L \rightarrow \infty} \alpha_{L}$ of the scaling variable.

A distribution is said to be broad if its moments $\left\langle X^{n}\right\rangle$ grow like exp $f(n)$ with $f(n)$ increasing stronger than linear with $n$, or if its moments might even diverge. This notion refers to the fact that the corresponding distribution of the sum of $N$ independent realizations of the variable $X$ will still deviate substantially from a Gaussian, even for very large $N$. According to this definition of broadness a weak case of a broad distribution is realized by the exponential distribution $(X \geq 0)$

$$
\mathcal{P}(X)=\frac{1}{\langle X\rangle} \exp (-X /\langle X\rangle)
$$

for which the moments grow as $\left\langle X^{n}\right\rangle \propto n ! \sim \exp (n \ln n)$. The prototype of a broad distribution is the log-normal distribution

$$
\mathcal{P}(X) d X=\frac{1}{\sqrt{2 \pi} \sigma} \exp \left[-\frac{1}{2 \sigma^{2}}(\ln X-\langle\ln X\rangle)^{2}\right] d \ln X
$$

the moments of which grow as $\left\langle X^{n}\right\rangle \propto \exp \left(\sigma^{2} n^{2} / 2\right)$. It represents the generic distribution for random multiplicative variables.

After these general remarks we would like to calculate the conductance distribution in higher dimensions including the LD transition. However, this is difficult. An interesting attack has been undertaken by Shapiro [116. He used the classical parallel composition law to derive Fokker-Planck equations for the conductance distribution in higher dimensions. However, the uncontrolled approximations involved overestimate fluctuations. Analytical approaches are usually restricted to dimension $d=2+\epsilon$ where the critical conductance is of order $\epsilon^{-1}$ and perturbation theory together with renormalization techniques can be used. We do not enter this technically involved subject and we will briefly review the main conclusions in Sects. VIII, IX.

Here, we will turn to local quantities and address the question of power law scaling relevant to the delocalized phase and the LD transition.

In the delocalized phase wavefunctions are extended all over the system. The correlation length $\xi_{c}$ in this phase can be estimated from

$$
g\left(\xi_{c}\right)=\mathcal{O}(1)
$$

Taking the metallic limit, Eq. (29), one finds that $(d>2)$

$$
\xi_{c} \sim\left(\frac{\lambda_{F}}{l_{e}}\right)^{\frac{1}{d-2}} l_{e}
$$

is a truly microscopic scale. Thus, one can expect a power law scaling of moments of the box probability $P\left(E, L_{b}\right)$ for $\xi_{c} \ll L_{b} \ll L$. In the critical regime of the LD transition the 
correlation length $\xi_{c}$ is larger than system size $\mathrm{L}$ and one can thus also expect a power law scaling of moments of the box probability $P\left(E, L_{b}\right)$ for $l \ll L_{b} \ll L \ll \xi_{c}$ (where $l$ stands for microscopic scales). Therefore, we will now consider distribution functions the moments of which display power law scaling. To simplify notations we consider a random variable $X \in[0,1]$ and a scale $\lambda \in[0,1]$ with distribution $\mathcal{P}(X ; \lambda)$. We assume that for sufficiently small $\lambda \ll 1$ the moments obey power law scaling

$$
\left\langle X^{q}\right\rangle_{\lambda}=\int_{0}^{1} d X \mathcal{P}(X ; \lambda) X^{q}=c_{q} \lambda^{\tilde{\tau}(q)}
$$

reflecting that scales in the system are separated. Here $c_{q}$ and $\tilde{\tau}(q)$ are treated as functions of arbitrary real values $q$.

The power law scaling condition is very restrictive and allows for a number of conclusions:

1. Normalization requires

$$
c_{0}=1, \quad \tilde{\tau}(0)=0
$$

2. Since $\tilde{\tau}(q)$ is independent of $\lambda$ one can calculate its derivatives and send $\lambda \rightarrow 0$. By this one can conclude two strong inequalities

$$
\tilde{\tau}^{\prime}(q) \geq 0, \quad \tilde{\tau}^{\prime \prime}(q) \leq 0
$$

where the prime means differentiation with respect to $q$.

3. Introducing the variable $Y=-\ln X \in[0, \infty[$ one can see that the moments are the Laplace transform of the function $e^{-Y} \mathcal{P}(X(Y) ; \lambda)$,

$$
\left\langle X^{q}\right\rangle_{\lambda}=\int_{0}^{\infty} d Y\left[e^{-Y} \mathcal{P}(X(Y) ; \lambda)\right] e^{-Y q}
$$

Thus, one may reconstruct the distribution from the moments by inverse Laplace transformation (if the latter can be carried out).

4. The case of strictly linear $\tilde{\tau}(q)=\gamma q(\gamma=0$ is not interesting) allows for a global rescaling of the distribution function by its first moment.

$$
\mathcal{P}(X ; \lambda)=\frac{1}{\lambda^{\gamma}} \tilde{\mathcal{P}}\left(\frac{X}{\lambda^{\gamma}}\right),\langle X\rangle_{\lambda}=c_{1} \lambda^{\gamma}
$$

and the coefficients $c_{q}$ are determined by the scale independent distribution $\tilde{\mathcal{P}}(Z)$

$$
c_{q}=\int_{0}^{1} d Z \tilde{\mathcal{P}}(Z) Z^{q} .
$$


A linear behavior of $\tilde{\tau}$ seems to be natural for power law scaling; all moments scale as powers of the first and only one critical exponent describes the scaling of all of them, including that of the distribution function. The rescaled distribution function is now scale independent and can take any form, e.g. it can be a broad distribution in the sense defined above. Examples are the Rayleigh and Porter-Thomas distributions found for the box-probability in the ideal delocalized phase (see Eqs. (140,141)). We refer to the situation of linear $\tilde{\tau}(q)$ as the gap scaling situation.

5. The linear case is not the most general one. For finite curvature in $\tilde{\tau}(q), \tilde{\tau}^{\prime \prime}(q)<0$, the coefficients $c_{q}$ can be treated as constant since the essential $q$ dependence that dominates for small $\lambda$ comes from $\tilde{\tau}(q)$. We will see in the following that the distribution function $\mathcal{P}(X, \lambda)$ can be essentially reconstructed from $\tilde{\tau}(q)$ by a Legendre transformation and it is an almost log-normal distribution with special features. One can write

$$
\mathcal{P}(X ; \lambda) d X=\mathcal{N} \lambda^{-\tilde{f}(a)} d a, \quad a:=\frac{\ln X}{\ln \lambda}
$$

where the normalization $\mathcal{N}$ has only a weak $\lambda$ dependence (e.g. $\sim \ln \lambda$ ). The essential information is now contained in the function $\tilde{f}(a)$ which is the Legendre transform of $\tilde{\tau}(q)$,

$$
\tilde{f}(a(q))=a(q) q-\tilde{\tau}(q), a(q):=\frac{d \tilde{\tau}(q)}{d q}>0
$$

Before we outline a derivation of the statement we make further comments.

6. In contrast to the gap scaling situation with arbitrary form of the scale independent distribution we have now the situation that the form of the distribution is fixed by a spectrum of scaling exponents. This situation has been phrased (in a bit more restricted context) multifractal scaling situation (for a review see [105]). The spectrum has several analytical properties following from the Legendre transformation. It has a unique maximum at $(a(0), 0)$ and a slope of 1 at $a(1)$. The derivatives fulfill

$$
\frac{d \tilde{f}}{d a}=q(a), \quad \frac{d^{2} \tilde{f}}{d a^{2}}=\frac{1}{\tilde{\tau}^{\prime \prime}(q(a))}<0 .
$$

Close to the typical value

$$
X_{\mathrm{t}}(\lambda)=\exp \left(\langle\ln X\rangle_{\lambda}\right) \propto \lambda^{a_{0}}, \quad a_{0}=a(0)
$$

the function $\tilde{f}(a)$ is well approximated by a parabola and $\mathcal{P}(X ; \lambda)$ is approximated by a log-normal distribution 


$$
\mathcal{P}(X ; \lambda) d X \approx \mathcal{N} \exp \left[-\frac{\ln ^{2}\left(X / X_{\mathrm{t}}\right)}{2 \sigma^{2}(-\ln \lambda)}\right] d \ln X
$$

where $\sigma^{2}=-1 / \tilde{\tau}^{\prime \prime}(0)$ is a positive constant.

The derivation of Eq. (192) relies on the fact that a finite curvature of $\tilde{\tau}(q)$ allows for a stationary point evaluation of the integral in Eq. (186) for each moment $\left\langle X^{q}\right\rangle_{\lambda}$ in the limit $\lambda \rightarrow 0$. Writing $X=\lambda^{a}$ and transforming to the variable $a$, with the distribution $\tilde{\mathcal{P}}(a, \lambda) d a=$ $\mathcal{P}(X, \lambda) d X$, yields the integral relation

$$
c_{q} \exp [(\ln \lambda) \tilde{\tau}(q)]=\int_{0}^{\infty} d a \exp [-\ln \lambda(F(a, \lambda)-a q)]
$$

with

$$
F(a, \lambda):=-\frac{\ln (\tilde{\mathcal{P}}(a, \lambda))}{\ln \lambda} .
$$

The function $F(a, \lambda)-a q$ can have a maximum with respect to $a$ (for $\lambda \ll 1$ ) that is only weakly dependent on $\lambda$. It yields a sharp maximum in the integrand (due to the large pre-factor $-\ln \lambda)$. Then, the integrand is determined up to $\mathcal{O}(1 /(-\ln \lambda))$ by the value of the integrand at this maximum and one finds that $\tilde{\tau}(q)$ is the Legendre transform of $F(a, \lambda)$. Consequently, $F(a, \lambda)$ must have a very weak $\lambda$ dependence (e.g. $\sim \ln |\ln \lambda|$ ). In case that $F(a, \lambda)-a q$ has no maximum with respect to $a$ that is only weakly dependent on $\lambda$ the corresponding $\tilde{\tau}(q)$ will not have finite curvature, but the gap scaling situation applies.

The phrase multifractality has its origin in the interpretation of $X(\lambda)$ as a local measure defined for a geometrical object embedded in a $d$-dimensional cube of linear size $L$. The scale $\lambda$ is given by the ratio $\left(L_{b} / L\right)^{d}$ where $L_{b}$ is the linear size of a box on which the measure is calculated. Due to normalization $X(1)=1$ the average of $X(\lambda)$ is the inverse of the number of non-empty boxes and thus yields, for $\lambda \ll 1$, the fractal dimension $D_{F}$ of the geometrical object

$$
\langle X\rangle_{\lambda} \propto\left(\frac{L_{b}}{L}\right)^{D_{F}}=\lambda^{D_{F} / d}
$$

The fact that higher moments $\left\langle X^{q}\right\rangle_{\lambda}$ may scale not simply as $\propto \lambda^{\left(D_{F} / d\right) q}$ but as $\propto$ $\lambda^{(1 / d)\left(D_{F}+\tau(q)\right)}$ where $\tau(q)$ defines a set of $q$-dependent generalized dimension $D(q)$ by $\tau(q)=:(q-1) D(q)$, has led to the notion of multifractality. Especially, one has $\tau(0)=-D_{F}$, $\tau(1)=0$. The Legendre transform of $\tau(q)$ then defines the so-called multifractal $f(\alpha)$ spectrum. It can be interpreted as the fractal dimension of that subset of the object where the measure scales locally as $\left(L_{b} / L\right)^{\alpha}$ (see [60). The general analytic properties of multifractal exponents are visualized in Fig. 22

We see that, for measures $X(\lambda)$, we have the following identification 


$$
\tilde{\tau}(q)=\frac{1}{d}\left(D_{F}+\tau(q)\right), \quad \tilde{f}(a)=\frac{1}{d}\left(-D_{F}+f(\alpha)\right), \quad a=\frac{\alpha}{d}
$$

Under quite general conditions, $\tilde{f}(a)$ (or $f(\alpha)$ ) is also bounded from below by $-D_{F} / d$ (or $\tau(1)=0$ ) and terminates with infinite slope at the endpoints of the finite interval $[a(\infty), a(-\infty)]$ (or $[\alpha(\infty), \alpha(-\infty)])$. In many cases the parabolic approximation of Eq. (196) extends to values $\alpha \geq \alpha(1)$ and the constraint on the slope of $f(\alpha)$ at $\alpha(1)$ allows to write a parabolic approximation (PA) for $f(\alpha)$ that depends only on one-parameter (besides $D_{F}$ ) $\alpha_{0}$

$$
f(\alpha)=D_{F}-\frac{\left(\alpha-\alpha_{0}\right)^{2}}{4\left(\alpha_{0}-D_{F}\right)} .
$$

For example, the box-probability $P\left(E ; L_{b}\right)$ considered in the LD-transition problem (Eq. (123)) is of this type of variable, since the squared amplitude of the wave function defines a local measure. The fractal dimension $D_{F}=d$ is always trivial. In the idealized delocalized phase we found the gap scaling class and $\tau(q)=(q-1) d$. The interesting information is contained in the shape of the corresponding scale independent distribution.

For the LD transition, however, it is known from a calculation by Wegner [133, 24] for $2+\epsilon$ dimensions that the multifractal scaling class applies and $D(q) \not \equiv d$. The PA he calculated yields

$$
\alpha_{0}-d=\epsilon=1 / g^{*}
$$

In summary, we found that the restriction to power-law scaling leads to two distinct classes of distribution functions. The gap scaling class is characterized by a single scaling exponent and a related scale independent distribution of arbitrary shape. The multifractal scaling class is characterized by a spectrum of scaling exponents and a shape of the distribution close to log-normal. The distribution of local box-probability in the ideal delocalized phase falls into the gap scaling class. The possibility of the multifractal scaling class is interesting for the LD transition point, because it opens a possibility to find an appropriate order parameter in terms of the LDOS. If, as it can happen in the multifractal scaling class, average and typical DOS scale differently with respect to system size, the inability of the average DOS to work as an order parameter must not be shared by the typical DOS. In Sect. IXB we will see that, indeed, this is the case.

\section{STATISTICS IN REALISTIC MODELS}

In this section we will review known facts about the statistics of conductance, energy levels and local quantities for the delocalized phase with large typical conductance $g \gg 1$ and for the localized phase with finite typical localization length $\xi \gg l_{e}$. The onset of broad 
distributions with log-normal tails in the delocalized phase is most interesting here. In 2D it leads to multifractality of the LDOS. In order to interpret this phenomenon we consider the conductance distribution for quasi-1D systems for which the number of channels is large, but the width is still of the order of a microscopic scale, e.g. the mean free path. Under these conditions a Fokker-Planck approach works that reproduces the UCF phenomenon, but the long tails are absent. We come to the conclusion that the log-normal tails are a precursor of the LD transition and do only occur if the relevant scaling variable is not infinite. To study more realistic quasi-1D systems the method of Finite Size Scaling (FSS) will be outlined. In this method a scaling variable is defined which is close to the definition of typical conductance.

\section{A. Onset Of Broad Distributions}

In the delocalized phase of a finite mesoscopic conductor where the average conductance is large $\langle g\rangle \gg 1$ the Büttiker formula tells that many $(\mathcal{O}(g))$ open modes are occupied. Thus, the conductance is a sum of many random numbers $\mathcal{T}_{i} \approx 1$. Naive application of the central limit theorem suggests that the conductance distribution will be a Gaussian centered around $\langle g\rangle$ with a relative deviation $\sqrt{\left\langle(\delta g)^{2}\right\rangle} /\langle g\rangle \sim 1 / \sqrt{\langle g\rangle}$ where $\delta g=g-\langle g\rangle$. However, this reasoning ignores the strong correlations between the transmission eigenvalues $\mathcal{T}_{i}$. This correlation leads to the occurrence of universal conductance fluctuations (UCF) (cf. Sec. [IB , i.e. the variance is $\left\langle(\delta g)^{2}\right\rangle \sim \mathcal{O}(1)$ and does neither depend on the actual value of $\langle g\rangle$ nor on the system size $L$. Taking UCF into account the conductance distribution seems to follow one-parameter scaling: it is Gaussian with scale dependent average value (which is also typical here) and a universal variance.

$$
\mathcal{P}(g ; L) \propto \exp \left[-\frac{\left(g-\langle g\rangle_{L}\right)^{2}}{2\left\langle(\delta g)^{2}\right\rangle}\right] .
$$

This was the common belief until the work of Al'tshuler et al. [5]. In this work it was shown by a combination of diagrammatic perturbation theory and renormalization group techniques that the conductance distribution deviates from a Gaussian for $|\delta g| \gg \sqrt{\langle g\rangle}$. For these tails the distribution crosses over to a log-normal form (see Fig. 23). This was concluded from the calculation of high moments which showed the characteristic dependence

$$
\left\langle(\delta g)^{n}\right\rangle \propto e^{u n^{2}} .
$$

Here $u \sim \mathcal{O}(1 /\langle g\rangle)$ indicating that a finite value of conductance is responsible for the effect.

The calculations were carried out for $2 \mathrm{D}$ (with localization lengths much larger than system size) and $d=2+\epsilon$ (in the delocalized phase). In the work by Al'tshuler et al. it was conjectured that their finding will penetrate to the LD transition point, i.e. that the conductance distribution will become very broad. 
The observations of these log-normal tails led to questioning the whole idea of oneparameter scaling. Later, Shapiro and coworkers [36] showed that these tails are not in conflict with one-parameter scaling theory as defined in Secs. VIIG. On the basis of the $2+\epsilon$ results they concluded that a critical conductance distribution will appear with long power-law tails. Such tails lead to divergence of low moments and exclude any one-parameter scaling theory in terms of moments of the conductance.

Later works 101,50,97 confirmed the calculation of Al'tshuler et al. and showed its principle validity for $3 \mathrm{D}$ as well. The $1 \mathrm{D}$ and quasi-1D cases need special attention since strong localization dominates.

In the localized phase the Büttiker formula tells that the conductance is dictated by the smallest value $\nu_{1}$ corresponding to eigenvalues of $M^{\dagger} M$ (cf. Eq. (86)). This value is known to be Gaussian distributed and thus it is no surprise that the conductance distribution, for $\xi \ll L, g \sim \exp (-\xi / L) \ll 1$, is of the log-normal form. This reflects the Gaussian distribution of inverse localization lengths. The typical localization length $\left(\left\langle\nu_{1}\right\rangle^{-1} 2 L\right)$ defines the typical conductance as the geometric mean $g_{\mathrm{t}}=\exp (\langle\ln g\rangle)$. Here, in principle a second parameter besides $g_{\mathrm{t}}$ might occur in the distribution, namely the variance of $\nu_{1}$. However, as happened already in the 1D case (cf. Sect. VIIQ), all known results indicate that this variance is simply related to the average value (cf. e.g. [91]) and thus only one-parameter seems to describe the distribution accurately,

$$
\mathcal{P}(g ; L) d g \propto \exp \left[-\frac{\left(\ln g-\langle\ln g\rangle_{L}\right)^{2}}{2 \sigma_{L}^{2}}\right] d \ln g,
$$

with

$$
\sigma_{L}^{2} \propto\langle\ln g\rangle_{L}
$$

The behavior of the conductance distribution close to the LD transition will be discussed in Sect. [XO.

Concerning the level statistics we restrict our discussion to the two-level correlation function $R(s)$. The energy separation $s \Delta$ can be associated with an inverse time scale. The behavior of $R(s)$ is related to the question how a wave packet evolves in time $\hbar /(s \Delta)$ [13],32]. For times larger than the diffusion time $t_{D}$ the wave packet explores the whole system several times and it is not surprising that here the standard random matrix theory results are approximately valid. In the standard random matrix theory for the idealized delocalized phase $R(s)$ only depends on the level distance $s$ measured in average level spacing units. A finite, but large, conductance is related to the diffusion time via the Thouless energy $E_{\mathrm{Th}}=h / t_{D} \gg \Delta$, the relevant transport energy scale. Consequently, the dimensionless conductance $g$ (here $g$ can be taken as the average value) sets a scale on the $s$-line. Indeed, one finds different level correlation functions for $s \ll g$ and for $s \gg g$. In the first case, as expected, the function $R(s)$ is approximately given by the standard random matrix theory 
result and only small corrections (proportional to powers of $1 / g$ ) occur. For example, the result for systems with broken time reversal symmetry reads [82]

$$
R(s)=-\left(\frac{\sin \pi s}{\pi s}\right)^{2}+\frac{A_{d}}{\pi^{2} g^{2}} \sin ^{2}(\pi s)
$$

( $A_{d}$ a constant depending only on dimension and symmetry).

The case $s \gg g$ has been investigated by Al'tshuler and Shklovskii [4] and the finding is (for $s$ not exceeding the inverse of the microscopic mean free time $\tau$ and possible oscillations on the scale $s \sim 1$ are ignored)

$$
R(s)=\frac{C_{d}}{g^{d / 2}|s|^{2-d / 2}}
$$

where $C_{d}$ is a numerical coefficient that depends only on $\mathrm{d}$ and on the symmetry class. The result can be understood by supposing diffusive motion of wave packets in the system.

In 2D there exists a broad cross over regime between the two asymptotic regimes of Eqs. (207,208) [81]. This can be understood as follows. The 2D metal resembles a critical system in as much as the corresponding $\beta$-function is close to 0 . The strong localization that will finally dominate comes along with very large localization lengths. It has to be expected that the level statistics will change on approaching the LD transition?]. A similar behavior can be expected to occur in the $2 \mathrm{D}$ case.

The level-correlation function for the localized phase with vanishing localization length is given by the Poisson statistics, Eq. (119), i.e. no-correlation for $s \neq 0$. To take a finite localization length into account which is, however, much smaller than the system size $L$, $\xi \ll L$, it is helpful to think of dividing the system into subsystems of volume $\xi^{d}$. The level-correlation function $\mathrm{R}(\mathrm{s} ; \mathrm{L})$ will then obey a scaling form [14, 3]

$$
R(s ; L)=\left(\frac{\xi}{L}\right)^{d} F\left(s(\xi / L)^{d}\right) .
$$

In the thermodynamic limit the level correlations vanish, $\lim _{L \rightarrow \infty} R(s, L)=0$, in accordance with the Poisson statistics being the limiting case.

The scaling form is in agreement with the one-parameter scaling theory since the variable $\xi / L$ is related to the typical conductance in the localized phase, $g_{t}=e^{-2 L / \xi}$.

The form of the scaling function $F(x)$ can be obtained for $x$ being large or small compared to 1 . For $x \gg 1$, the corresponding wave-packet dynamics probes only the region within one localization volume and is, thus, diffusive. Consequently one recovers the Al'tshulerShklovskii behavior [3]

\footnotetext{
${ }^{5}$ We will discuss some of the consequences in Sect. IX
} 


$$
F(x) \propto x^{-(2-d / 2)} .
$$

For $x \ll 1$ but $|\ln x|<L / \xi$ regions much larger than one localization volume are probed and the only correlation between levels comes from weak couplings between such volumes [3] resulting in a logarithmic dependence on $x$

$$
F(x) \propto(\ln x)^{d} .
$$

We now turn to the statistics of local quantities. Again, for a good metal with $g \gg$ 1 the distribution of local quantities is essentially given by the standard random matrix theory (cf. Eqs. (140,141)). However, the long tails are different and the finite value of $g$ becomes important. We concentrate on the box-probability $P\left(E ; L_{b}\right)$ in the following. This generalizes to the reduced $\operatorname{LDOS} \tilde{\rho}\left(E ; L_{b}\right)$. We introduce the normalized quantity

$$
t:=\frac{P(E ; l)}{\langle P(E)\rangle_{l / L}},\langle P(E)\rangle_{l / L}=(l / L)^{d}
$$

and consider the case $g \gg 1$. The scale $l$ stands for microscopic box size. The threshold for the standard random matrix result turns out to be $t \sim 1 / \sqrt{g}$. For $t \ll 1 / \sqrt{g}$ the Rayleigh and Porter-Thomas distributions are valid up to small corrections of $\mathcal{O}(1 / g)$ (cf. [98]).

For $t \gg 1 / \sqrt{g}$ a number of authors [101,50,97, 120] have found the onset of broad distributions which in 2D and 3D develop long tails of the form

$$
\mathcal{P}(t) \sim \exp \left(-A_{d} \ln ^{B_{d}}\left(t / t_{d}\right)\right) .
$$

Here $A_{d}$ depends on the dimensionality $d$ and on the disorder strength and $B_{2}=2$. For $B_{3}$ values of 2 and 3 have been predicted. The value $t_{d}$ is a typical value serving as reference point. The precise expressions are currently under debate (because the authors of [101,50, 97, 120 use different approximative calculation schemes). However, what seems to be clear is the occurrence of long-tails of the general form Eq. (213). For the 2D case there is also agreement that $A_{2} \propto g /(\ln L / l)$ and also $t_{d} \propto g^{-1} \ln (L / l)$. Due to this scaling behavior the result can (for $l \approx l_{e}$ ) be identified with a multifractal distribution within the parabolic approximation (PA, cf. Eq. (201))

$$
\mathcal{P}(t) \sim \exp \left(-\frac{\left(\ln t-\left(\alpha_{0}-2\right) \ln (l / L)\right)^{2}}{-\ln (L / l) 4\left(\alpha_{0}-2\right)}\right)
$$

where the essential parameter $\alpha_{0}-d$ is here $(\mathrm{d}=2)$ proportional to the inverse of the conductance

$$
\alpha_{0}-d \propto g^{-1} .
$$

Thus, states in a good 2D metal (with the localization length being much larger than the system size) are multifractally distributed. Since the metallic situation in 2D resembles a 
critical regime of a LD transition the finding is, after Wegner's pioneering work 133 and the work by Al'tshuler et al. [5] a further analytical hint at the multifractality of critical eigenstates.

The case of $1 \mathrm{D}$ and quasi-1D conductors need special attention. In $1 \mathrm{D}$ the localization length $\xi$ is of the order of the mean free path $\xi \approx l_{e}$ and thus there is no room for diffusive behavior. For the quasi-1D case one has to be a bit more precise in fixing the sizes. The length $L$ is treated variable and the cross-section $L_{t}^{d-1}$ is kept fixed. However, the dimensionality is in general essential for the determination of physical quantities such as the localization length. A strong simplification arises for the case when $L_{t} \approx l_{e}$ but a huge number $N_{c} \gg 1$ of channels is occupied. Under these conditions the dimensionality becomes inessential and the only kinematic quantity besides $L$ is the channel number $N_{c}$. We refer to this case as the idealized quasi-1D case. For the idealized quasi-1D system one can show that

$$
\xi\left(N_{c}\right)=A N_{c} l_{e}
$$

with $A$ a numerical constant of order 1 . We have anticipated this result already in Sect. $\nabla \mathrm{B}$ and will sketch a more reliable derivation in the following subsection. The fact that one can reach a metallic regime by

$$
l_{e} \ll L \ll \xi\left(N_{c}\right)
$$

makes the idealized quasi-1D system more interesting than strictly 1D. On the other hand, the idealized quasi-1D system is still tractable by analytical methods (cf. e.g. 121,58).

In metallic idealized quasi-1D systems one has an average conductance (which is also typical)

$$
g=A^{\prime} \xi / L, \quad A^{\prime} \sim \mathcal{O}(1)
$$

The distribution of $t$ is, for $t \ll 1 / \sqrt{g}$, again given by the standard random matrix theory with small corrections of $\mathcal{O}(1 / g)$ (cf. 98]). However, for $t \gg 1 / \sqrt{g}$ the situation is different as compared to the 2D and 3D case. One finds no log-normal tails but stretched exponential tails (at least when the box is shrinking to a point)

$$
\mathcal{P}(t) \sim \exp (-B \sqrt{C t g}), B, C \sim \mathcal{O}(1)
$$

Note, that $g=A \xi / L$ is the quasi-1D conductance.

In the localized regime of idealized quasi-1D systems, $\xi \ll L$, where the typical conductance is $g \propto e^{-2 L / \xi}$, one finds a similar behavior for $t \gg(\xi / L)^{-1}$

$$
\mathcal{P}(t) \sim \exp (-B \sqrt{C t(\xi / L)})
$$

Note, that $\xi / L$ is related to the typical conductance here. Both equations, (219,220), have $\xi / L$ as the reference point. In order to have $t \gg(\xi / L)^{-1}$ one has to have $\xi \gg l_{e}$. Thus, 
the stretched exponential tail does occur when the box-size is much less the localization length. In the case where the box-size becomes much larger than the localization length the previously obtained results of the idealized localized phase apply (cf. Sect. VIA).

The tails of the distribution $\mathcal{P}(t)$ that have been observed in idealized quasi-1D (stretched exponential), in 2D (log-normal) and in 3D (log-cube) have all been interpreted in terms of anomalously localized states (or pre-localized states) [5. 101,98. The notion refers to anomalously rare states with sharp peak in the amplitude on top of an extended backround (in 3D delocalized phases) or localized states with anomalously short localization radius (in $d \leq 2$ ). The question arises what the tails might say in view of the LD transition. The 2D case seems to be clear. Here the log-normal tails correspond to multifractal scaling to be expected once the correlation length is much larger than system size. This interpretation is in accordance with the finding of $\log$-normal tails in $d=2+\epsilon$. The distributions become multifractal when the conductance is close to its critical value. In $2 \mathrm{D}$ the correlation length is the localization length which is much larger than the system size in the $g \gg 1$ regime, and thus the states are critical in the sense that the system size is less the correlation length. In $3 \mathrm{D}$ with $g \gg 1$ the correlation length is (see Eq. (185)) microscopic and there is no reason to expect multifractal scaling. In quasi-1D with $g \gg 1$ the quasi-1D localization length is much larger than the system length $L$, however due to the strong difference between $L_{t} \approx l_{e}$ and $L$, this length cannot be considered as the correlation length. In strict $1 \mathrm{D}$ the correlation length is the 1D localization length which is again microscopic and no critical behavior of eigenstates can be expected on scales larger than $l_{e}$.

In this context it is worthwhile to discuss the findings about the LDOS for open systems. These are defined by surrounding the system with an ideally conducting medium. In that case we cannot talk about eigenstates of the system. Still the LDOS as given by the imaginary part of the Green's function is a meaningful object. The system itself does not conserve total probability which formally breaks hermiticity of the effective Hamiltonian for the system. For the 2D and 3D case, however, the results for the distribution of LDOS are qualitatively the same as for the distribution of the restricted LDOS in the closed systems. A qualitative difference occurs for the quasi-1D case. In the open quasi-1D conductor the LDOS shows log-normal tails,

$$
\mathcal{P}(\rho) \sim \exp \left(-A g \ln ^{2}(\rho / \nu)\right),
$$

where $\nu$ is the average DOS, $A$ is of order 1 , and $\rho / \nu \gg 1$. This equation is also valid in strict 1D. The log-normal tails are not due to the statistics of the eigenstates of the corresponding closed system, but can rather be interpreted as reflecting the distribution of current-relaxation times for the open system. The latter have a similar log-normal distribution. The broadness of this distribution is due to the presence of anomalously long-living states 98.

In order to better understand the idealized quasi-1D system we will focus in the following 
subsection on the description of transport in idealized quasi-1D systems. One particular question we like to address [29]: is it possible to obtain similar log-normal tails for the conductance distribution in the metallic regime as those found in the $d=2+\epsilon$ case by Al'tshuler et al. [5]?

\section{B. Statistics in Quasi-One-Dimension}

The transport properties of a quasi-1D system can be obtained within a transfer matrix modeling as outlined in Sect. VA. The characteristic parameters are the length $L$, the number of channels $N_{c}$ related to the cross-section $L_{t}^{d-1}$ and the mean free path $l_{e}$.

In close analogy to the procedure that led to a FPE for the intrinsic resistance in 1D (Eq. (174) one can derive a FPE for the whole transfer matrix $M$. The essential assumptions for this to be possible are:

1. The locally weak scattering condition

$$
\frac{1}{l_{e}}=\lim _{\delta L \rightarrow 0} \frac{N_{c}^{-2} \sum_{\alpha \beta}\left\langle R_{\alpha \beta}(\delta L)\right\rangle}{\delta L} .
$$

2. The statistical independence of strip transfer matrices defined for adjacent strips.

Fixing the statistical properties of a single strip transfer matrix yields a FPE for $\mathcal{P}(M ; L)$. One particular model turned out to be analytically tractable to a large extent, the socalled isotropic quasi-1D model. In this model [42] it is assumed that forward and backward scattering within a strip $S$-matrix is equal for all channels. This crucial simplification means that there is no diffusion in transversal direction. The modeling thus restricts the width $L_{t}$ to be less than the mean free path $l_{e}$ [93. Therefore, an idealized quasi-1d model in the sense of the foregoing section results. Furthermore, the most explicit results can be obtained in the limit of large channel numbers, or more precisely when $N_{c} \rightarrow \infty$ but $N_{c} l_{e} / L$ remaining fixed and finite.

In terms of the polar parameterization Eq.(87) the isotropy assumption is equivalent to the assumption that the unitary matrices $u^{i}$ are distributed isotropically within the unitary group. Similar to the standard random matrix theory for Hamiltonians the statistics of eigenvectors is treated as being trivial and decoupled from the statistics of the radial parameters $\lambda_{i}$.

A consequence of the isotropy assumption is the fact that a closed FPE can be found for the distribution of the radial parameters $\lambda_{i}$ alone. These radial parameters determine the conductance (Eq. (86))

$$
g=\sum_{i} \mathcal{T}_{i}=\sum_{i} \frac{1}{1+\lambda_{i}} .
$$


The derivation follows the same principle steps as outlined in Sect. VII and the final result is the well-known DMPK-equation 42]

$$
\begin{aligned}
l_{e} \frac{\partial \mathcal{P}\left(\left\{\lambda_{i}\right\}\right)}{\partial L} & =\frac{2}{\gamma} \sum_{i} \frac{\partial}{\partial \lambda_{i}} \lambda_{i}\left(1+\lambda_{i}\right) J \frac{\partial}{\partial \lambda_{i}} J^{-1} \mathcal{P}\left(\left\{\lambda_{i}\right\}\right), \\
J & =\prod_{n<m}\left|\lambda_{m}-\lambda_{n}\right|^{\beta}, \quad \gamma=\beta N_{c}+2-\beta .
\end{aligned}
$$

Here $\beta=1,2,4$ characterizes different symmetry classes (systems with time reversal symmetry $\beta=1$, systems with broken time reversal symmetry $\beta=2$, systems with time reversal symmetry and spin-orbit interaction $\beta=4$ ) and $J$ is the Jacobian for the transformation from the Cartesian parameterization $M_{k l}$ to the polar parameterization of the transfer matrix.

The DMPK equation has been solved exactly by Beenakker and Rejaei [17] for $\beta=2$ and for $\beta=1,4$ formal solutions exist [23]. Common to the solutions is that they represent a Gibbs-ensemble of the form already encountered in the standard random matrix theory for energy levels, Eqs. (133,134),

$$
\begin{aligned}
\mathcal{P}\left(\left\{\lambda_{i}\right\}\right) & =\exp \left(-\beta \mathcal{H}\left(\left\{\lambda_{i}\right\}\right)\right) \\
\mathcal{H}\left(\left\{\lambda_{i}\right\}\right) & =\frac{1}{2} \sum_{m \neq n} U\left(\lambda_{n}, \lambda_{m}\right)+\sum_{i} V\left(\lambda_{i}\right) .
\end{aligned}
$$

For $\beta=2$ the explicit form of the two- and one-body potentials read

$$
\begin{aligned}
U(x, y) & =-\frac{1}{2} \ln (|x-y|)-\frac{1}{2} \ln \left(\left|\operatorname{arsinh}^{2} \sqrt{y}-\operatorname{arsinh}^{2} \sqrt{x}\right|\right), \\
V(x) & =\frac{N_{c} l_{e}}{2 L} \operatorname{arsinh}^{2} \sqrt{x}+\mathcal{O}\left(1 / N_{c}\right) .
\end{aligned}
$$

It is worth mentioning that, for $\lambda_{i} \ll 1$ (open channels $\mathcal{T}_{i} \approx 1$ ), the two-body potential reduces to

$$
U(x, y)=-\ln (|x-y|)
$$

which has been used to model quasi-1d wires, before the exact solution was found (see e.g. [121]). A general feature of the Gibbs-ensembles is that physical parameters, such as $N_{c}, l_{e}$ and $L$ do only occur in the one-body potential $V(x)$ in the combination $N_{c} l_{e} / 2 L$. In contrast, the two-body potential is a universal function reflecting universal level repulsion.

Without solving the DMPK equation explicitly one can study the resulting equations for the moments $\left\langle g^{n}\right\rangle$ by integrating on both sides of the DMPK equation over $\left(\sum_{i}\left(1+\lambda_{i}\right)^{-1}\right)^{n}$. A perturbative analysis as a series expansion in powers of the single parameter $N_{c} l_{e} / L$ is possible in the limit $N_{c} l_{e} \gg L \gg l_{e}$, i.e. in the diffusive limit (Mello et al. in Ref. [42]). This yields the result for the average conductance

$$
\langle g\rangle=N_{c} l_{e} / L+C_{\beta}+\mathcal{O}\left(\left(N_{c} l_{e} / L\right)^{-1}\right)
$$


The constant $C_{\beta}$ contains corrections to the classical result (for $\beta=1$ these are negative weak localization corrections). Thus, the leading term is the one we have already anticipated in Eq. (95). The leading term for the variance $\left\langle(\delta g)^{2}\right\rangle$ turns out to be universal

$$
\left\langle(\delta g)^{2}\right\rangle=\frac{2}{15 \beta}
$$

and proofs the UCF effect for isotropic quasi-1D. The effort to derive results for higher moments by the perturbative method increases drastically.

In the limit $L \gg N_{c} l_{e}$ it is advantageous to transform to the parameters $\nu_{i}$ (Eq. (86)) since they become the Lyapunov exponents in the thermodynamic limit. Their interaction can then be neglected and one ends up with an effective joint probability density of the form 89

$$
\mathcal{P}\left(\left\{\nu_{i}\right\}\right)=C_{\beta, N_{c}} \prod_{i=1}^{N} \exp \left[-\frac{\left(\beta N_{c}+2-\beta\right) l_{e}}{2 L}\left(\nu_{i}-\frac{L\left(\beta N_{c}+1-\beta i\right)}{l\left(\beta N_{c}+2-\beta\right.}\right)^{2}\right]
$$

demonstrating again the self-averaging feature of Lyapunov exponents. In that limit the conductance is $g=2 e^{-\nu_{1}}$ since all higher $\nu_{i}$ contribute much less. The conductance distribution is then found to be of the log-normal form. The typical value and the log-variance are related by $\left(N_{c} \gg 1\right)$ 甲

$$
\left\langle(\delta \ln g)^{2}\right\rangle=-2 \ln g_{\mathrm{t}}=-2\langle\ln g\rangle=\frac{4 L}{N_{c} l_{e} \beta} .
$$

The localization length is then found to be

$$
\xi=\beta N_{c} l_{e}
$$

Before we look at the conductance distribution in more detail we report on the findings concerning the distribution of transmittances $T_{\alpha \beta}=\left|t_{\alpha \beta}\right|^{2}$ and $T_{\alpha}=\sum_{\beta} T_{\alpha \beta}$.

They are related to the eigenvalues $\mathcal{T}_{i}=\left(1+\lambda_{i}\right)^{-1}$ of $t^{\dagger} t$ by unitary matrices $u$ and $v$

$$
T_{\alpha \beta}=\sum_{k l} u_{\alpha k} u_{\alpha l}^{*} \sqrt{\mathcal{T}_{k} \mathcal{T}_{l}} v_{k \beta} v_{l \beta}^{*}, \quad T_{\alpha}=\sum_{l}\left|u_{l \alpha}\right|^{2} \mathcal{T}_{l}
$$

These equations point out that transmittances are analogs of wave function amplitudes $\left|\psi_{\alpha}(k)\right|^{2}=\left|U_{k \alpha}\right|^{2}$. However, the transmittances do depend also explicitly on the eigenvalues $\mathcal{T}_{l}$. The statistics of the unitary matrices was assumed to be isotropic in the DMPK approach and the eigenvalues follow the DMPK equation.

\footnotetext{
${ }^{6}$ Notice, that the so-called global transfer matrix approach 108,121 yields a factor 1 between variance and average value instead of a factor 2 occuring in Eq. (231).
} 
It turned out that the distribution of transmittances can be calculated exactly 86. Actually, only $\mathcal{P}\left(T_{n}\right)$ needs to be calculated in the large $N_{c}$ limit. In a work by Kogan and Kaveh [78] it was shown that, for isotropic scattering in the large $N_{c}$ limit, the distributions of $T_{\alpha}$ and $T_{\alpha \beta}$ are connected by

$$
\mathcal{P}\left(T_{\alpha \beta}\right)=\int_{0}^{\infty} d T_{\alpha} T_{\alpha}^{-1} \exp \left(-T_{\alpha \beta} / T_{\alpha}\right) \mathcal{P}\left(T_{\alpha}\right)
$$

In the metallic regime $N_{c} l_{e} \gg L,\langle g\rangle \gg 1$, the distribution of $T_{\alpha \beta}$ is of the Rayleigh type for the bulk of the distribution $(\beta=2)$ and develops stretched exponential tails (first obtained by Nieuwenhuizen and van Rossum [102])

$$
\mathcal{P}\left(T_{\alpha \beta}\right) \sim \exp \left(-2 \sqrt{T_{\alpha \beta}}\right), T_{\alpha \beta} \gg\left(N_{c} l_{e} / L\right)^{2}
$$

It is therefore completely analogous to the distribution of wave function amplitudes in closed quasi-1D systems (see Eq. (219)). The corresponding distribution of $T_{\alpha}$ is Gaussian in the bulk and develops exponential tails

$$
\mathcal{P}\left(T_{\alpha}\right) \sim \exp \left(-T_{\alpha}\right), T_{\alpha} \gg N_{c} l_{e} / L
$$

These results depend heavily on the isotropy assumption and they indicate that it is very unlikely that the distribution of conductance, $g=\sum_{\alpha} T_{\alpha}$, in the metallic regime can develop log-normal tails in isotropic quasi-1D. However, so far the extension to conductance is not easy since the correlation between eigenvalues $\mathcal{T}_{i}$ have to be taken into account.

In the localized regime $L \gg N_{c} l_{e}=\xi / \beta$, the statistics is dominated again by the smallest value $\nu_{1}$ of $\nu_{i}$, i.e. by the largest value $\mathcal{T}_{1}$ of $\mathcal{T}_{i}$. This value is log-normally distributed (see Eq. (231)) and accordingly the transmittances are log-normally distributed, too.

Since the conductance is a linear statistics of the transfer matrix radial coordinates $\lambda_{i}$

$$
g=\sum_{i} f\left(\lambda_{i}\right), \quad f(x)=(1+x)^{-1}
$$

the problem of a complete statistical description of $g$ reduces to the problem of a linear statistics $X=\sum_{i} f\left(x_{i}\right)$ in a Gibbs-ensemble of a classical gas determined by a universal two-body potential (which can be chosen to be symmetric) and a parameter-dependent one-body (confining) potential

$$
\begin{aligned}
\mathcal{P}(X) & =\int d^{N_{c}} x \mathcal{P}\left(\left\{x_{i}\right\}\right) \delta\left(X-\sum_{i} f\left(x_{i}\right)\right), \mathcal{P}\left(\left\{x_{i}\right\}\right)=Z^{-1} \exp -\beta \mathcal{H}\left(\left\{x_{i}\right\}\right) \\
\mathcal{H}\left(\left\{x_{i}\right\}\right) & =\frac{1}{2} \sum_{m, n} U\left(x_{n}, x_{m}\right)+\sum_{i} V\left(x_{i}\right)
\end{aligned}
$$

Beenakker pointed out [16] that the one-body potential can be viewed as a source term in the partition sum 


$$
Z[V]=\int d^{N_{c}} x \exp \left[-\beta \mathcal{H}\left(\left\{x_{i}\right\}\right)\right]
$$

All cumulants of the level-density, $\rho(x):=\sum_{i} \delta\left(x-x_{i}\right)$, can be obtained by functional derivatives

$$
\left\langle\left\langle\rho\left(x_{1}\right) \ldots \rho\left(x_{k}\right)\right\rangle\right\rangle=\frac{\delta^{k} \ln Z}{\delta\left(-\beta V\left(x_{1}\right)\right) \ldots \delta\left(-\beta V\left(x_{k}\right)\right)} .
$$

Cumulants of a linear statistics $X=\sum_{i} f\left(x_{i}\right)$ are given by integration

$$
\left\langle\left\langle X^{k}\right\rangle\right\rangle=\int d x_{1} \ldots d x_{k}\left\langle\left\langle\rho\left(x_{1}\right) \ldots \rho\left(x_{k}\right)\right\rangle\right\rangle f\left(x_{1}\right) \ldots f\left(x_{k}\right) .
$$

The whole distribution $\mathcal{P}(X)$ can be obtained from a modified partition sum $Z(\kappa)$,

$$
\mathcal{P}(X)=\int d \kappa e^{i \kappa X} Z(\kappa)
$$

where $Z(\kappa)$ follows from $Z$ through a simple shift in the one-body potential

$$
V_{\kappa}(x):=V(x)+i \frac{\kappa}{\beta} f(x) .
$$

In order to work with functional derivatives one has to know $Z$ (or $Z(\kappa)$ ) as an explicit functional of the one-body-potential. Alternatively, the knowledge of the average level density $\nu(x):=\langle\rho(x)\rangle$ as a functional of the one-body-potential would be enough.

Such representation of the average level-density $\nu(x)$ was developed by Dyson [43]. Here we outline a derivation 29 which allows for a systematic check of the range of validity of the results.

We start from the partition sum $Z$, or $Z(\kappa)$ and proceed by the following steps: 1 . The Hamiltonian is expressed by the level-density $\rho(x)$, replacing sums by integration. Here the absence of self-interaction is ignored, i.e $\sum_{n \neq m} U\left(x_{n}, x_{m}\right)$ is replaced by $\int d x d y \rho(x) \rho(y) U(x, y)$. 2 . With the help of a $\delta$-functional we introduce a field $\phi(x)$ that takes the role of the leveldensity. 3. We represent the $\delta$-functional by its Fourier representation which introduces a conjugate field $\psi(x)$. 4. Now the original integration over the set $\left\{x_{i}\right\}$ can be carried out leaving a field theoretical partition function in terms of two field degrees of freedom, $\phi(x)$ and $\psi(x)$. 5. Due to the two-body character of the original $\mathcal{H}$ the field $\phi(x)$ can be integrated out by a Gaussian integration.

\footnotetext{
${ }^{7}$ Cumulants $\left\langle\left\langle X^{n}\right\rangle\right\rangle$ are linear combinations of moments of order $k \leq n$. While moments can be generated from a partition sum $Z$, the corresponding cumulants are generated by $\ln Z$. The Gaussian distribution is characterized by vanishing cumulants for $n \geq 3$
} 
The final result is a path integral representation of the partition sum $Z$ or of the complete distribution function $\mathcal{P}(X)$, where the integration runs over field configurations of $\psi(x)$. We concentrate on $\mathcal{P}(X)$ which reads

$$
\begin{aligned}
\mathcal{P}(X) & =\int D[\psi] \exp -S[\psi ; X] \\
S[\psi ; X] & =\frac{-1}{2 \beta}(\psi|K| \psi)+\frac{1}{2}(f|K| f) Q^{2}(\psi ; X)+F_{N_{c}}[\psi+\beta V] .
\end{aligned}
$$

Here we use a scalar product notation,

$$
(f|A| g):=\int d x d y f(x) A(x, y) g(y), \quad(f \mid g):=\int d x f(x) g(x),
$$

$K$ denotes the inverse operator of $U(U(x, y)=(x|U| y))$, the functional $Q(\psi ; X)$ is defined as

$$
Q(\psi ; X):=\frac{X-\beta^{-1}(f|K| \psi)}{\beta^{-1}(f|K| f)}
$$

and the functional $F_{N_{c}}$ is a free energy of $N_{c}$ independent particles with one-body-potential $\psi+\beta V$,

$$
F_{N_{c}}[\psi+\beta V]:=-N_{c} \ln \left[\int d x \exp [-(\psi(x)+\beta V(x))]\right] .
$$

The omission of the self-interaction can be cured on an effective potential level. By shifting the one-body-potential $V(x)$ to $\tilde{V}(x)=V(x)-\frac{1}{2} U(x, x+\Delta(x))$ where $\Delta(x)$ is the average level spacing at $x$, the theory is able to account for those quantities that are smooth on the scale of $\Delta(x)$. For example, with the logarithmic interaction, $U(x, y)=-\ln |x-y|$ the effective potential reads $\tilde{V}(x)=V(x)-\frac{1}{2} \ln \nu(x)$. In the following, we will denote $\tilde{V}$ by $V$.

The path integral in Eq. (244) cannot be calculated exactly. However, for large $N_{c}$ one can use the method of stationary point. To be more explicit we will now use the conductance $g$ as linear statistics variable and recall that $V(\lambda)$ contains the pre-factor $g_{0}=N_{c} l_{e} / L$ which we assume to be large: $g_{0} \gg 1$ since we are looking for the conductance distribution in the metallic regime.

Introducing the mean-field level density $\nu_{g}^{0}$ as

$$
\nu_{g}^{0}:=\frac{N}{Z_{g}^{0}} \exp \left[-\left(\psi_{g}^{0}+\beta V\right)\right], \quad Z_{g}^{0}:=\int d \lambda \exp \left[-\left(\psi_{g}^{0}(\lambda)+V(\lambda)\right)\right]
$$

corresponding to the stationary point,

$$
\left.\frac{\delta S}{\delta \psi}\right|_{\psi_{g}^{0}}=0
$$


the mean-field equation reads $\$$

$$
\left.\left.\left.\mid \nu_{g}^{0}\right)=-\tilde{K} \mid V+\beta^{-1} \ln \nu_{g}^{0}\right)+\beta^{-1} Q(g) \tilde{K} \mid f\right)+\frac{\left.N_{c} K \mid 1\right)}{(1|K| 1)} .
$$

Here the kernel $\tilde{K}$ is defined as

$$
\left.\tilde{K}=K-\frac{K \mid 1)(1 \mid K}{(1|K| 1)}, \tilde{K} \mid 1\right)=(1 \mid \tilde{K}=0,
$$

and $Q(g)$ as

$$
Q(g):=\frac{g-\bar{g}_{g}}{\beta^{-1}(f|\tilde{K}| f)}
$$

with

$$
\bar{g}_{g}:=-\left(f|\tilde{K}| V+\beta^{-1} \ln \nu_{g}^{0}\right)+\frac{N_{c}(f|K| 1)}{(1|K| 1)} .
$$

In deriving these equations it has been used that the mean-field level density is normalized $\left(1 \mid \nu_{g}^{0}\right)=N_{c}$ and yields the current $g$ as an expectation value $\left(f \mid \nu_{g}^{0}\right)=g$.

Now one can draw the following conclusions:

1. For $|V(\lambda)| \gg \beta^{-1} \ln \nu_{g}^{0}$ the expression $\bar{g}_{g}$ equals the average value of $g$, independently of current $g$. Since $V$ contains the large factor $g_{0}$ the inequality is satisfied as long as

$$
|\delta g| \ll\langle g\rangle,\langle g\rangle \gg 1
$$

2. The stationary point of $S$ is then given by

$$
S\left[\psi_{g}^{0} ; g\right]=\frac{1}{2} \frac{(g-\langle g\rangle)^{2}}{\beta^{-1}(f|\tilde{K}| f)}+S_{\langle g\rangle}
$$

where $S_{\langle g\rangle}$ is independent of current $g$.

3. One can also analyze fluctuations around the stationary point and show that they give sub-leading contributions to the path integral. Finally, one arrives at the conclusion, that the distribution of conductance is, for $|\delta g| \ll\langle g\rangle$,

$$
\mathcal{P}(g ; L)=\text { const. } \exp \left[-\frac{\left(g-\langle g\rangle_{L}\right)^{2}}{2 \beta^{-1}(f|\tilde{K}| f)}\right]
$$

up to $\mathcal{O}(\ln g)$ corrections in the exponent.

\footnotetext{
${ }^{8}$ This mean-field equation is in agreement with the one obtained by Dyson [43] and Beenakker [16] for the special two-body-potentials used in that works.
} 
Therefore, our conclusion is that the conductance distribution in isotropic quasi-1D does not show long-tails in the regime $|\delta g| \ll\langle g\rangle$, but is Gaussian with universal variance. The universality is due to the universal two-body potential $U(x, y)$ which reflects essentially the universal level repulsion expressed by the Jacobian in Eq. (224). Recall, that the occurrence of log-normal tails in $d=2+\epsilon$ happened already for $|\delta g| \gg \sqrt{\langle g\rangle}$.

The absence of log-normal tails in the conductance distribution for isotropic quasi-1D can be interpreted as being caused by the isotropy assumption. Relaxing from the isotropy assumption the resulting FPE for the radial parameters $\lambda_{i}$ is not closed. Rather the diffusion function depends on the statistics of eigenvectors of $M^{\dagger} M$. The latter is presently unknown, but one can conclude in general [30] that long tails in the distribution of the eigenvectors will lead to long-tails in the distribution of $g$. In addition, one can conclude that in such case the probability density of the radial parameters is no longer given by a Gibbs-ensemble with only one-body and two-body potentials. Also the results for transmittances indicate that the isotropy assumption expels any log-normal tails for the conductance distribution.

On the other hand, the isotropy assumption can only be justified for transversal width $L_{t}$ being less the mean free path $l_{e}$. It is instructive to look at the correlation length $\xi_{c}$ relevant to such idealized quasi-1D systems. Recall, that the correlation length, in the spirit of one-parameter scaling theory, is defined as that fictitious system size $\xi_{c}$ for which the conductance of a $d$-dimensional cubic system is of order 1 . To attach a correlation length to the quasi-1D system requires a meaningful definition of a conductance in a cubic system. The easiest way is to use a parallel composition law and define

$$
g_{\text {cube }}(L)=g_{\text {qua }}\left(L, L_{t}\right)\left(L / L_{t}\right)^{d-1}
$$

where $g_{\text {qua }}(L)$ is the conductance of the quasi-1D system with length $L$ and cross-section $L_{t}^{d-1}$. For the isotropic quasi-1D system with $g_{\text {qua }}(L) \approx N_{c} l_{e} / L$ and $L_{t}=A l_{e}, A \leq 1$, we thus have

$$
g_{\text {cube }}(L) \approx N_{c}\left(\frac{L}{l_{e}}\right)^{d-2}
$$

which is extremely large. Consequently, the correlation length is

$$
\xi_{c} \approx l_{e} N_{c}^{-1 /(d-2)} .
$$

Thus, even for $d=2$ the isotropic quasi-1D model corresponds to microscopic correlation lengths. One should note that it is not the quasi-1D localization length $\xi=\beta N_{c} l_{e}$ which plays the role of the correlation length, but $\xi_{c}$. Both coincide only for strict $1 \mathrm{D}$ where $N_{c}=1$.

We interpret the occurrence of log-normal tails in the conductance distribution as precursors of the LD transition. For $d=2+\epsilon$ they are controlled by the one-parameter scaling variable $g_{t}$. With this interpretation the absence of such tails in isotropic quasi-1D is no surprise since the appropriate scaling variable $\propto N_{c}\left(L / l_{e}\right)^{d-2}$ is sent to infinity. 


\section{Finite Size Scaling}

Giving up the isotropy assumption allows to study more realistic systems by a quasi1D set-up. True dimensionality effects should enter the physical properties. An analytical treatment becomes much more difficult, but a numerical treatment turns out to be very efficient. The Green's function can be studied by recursive methods [90], putting strips of cross-section $L_{t}^{d-1}$ together. Alternatively, strip transfer matrices can be multiplied [107]. That quantity which can be obtained with high precision (due to its self-averaging property) is the quasi-1D localization length $\xi\left(L_{t}\right)$. By studying $\xi\left(L_{t}\right)$ as a function of $L_{t}$ one should be able to study the localization behavior in a large (cubic) $d$-dimensional system.

Our first task is to construct a suitable scaling variable which can be derived from $\xi\left(L_{t}\right)$. When we increase $L_{t}$ and the system flows towards the insulating (localized) phase, $\xi\left(L_{t}\right)$ will stop growing as soon as $L_{t}$ is comparable with the (finite!) localization length of the infinite $d$-dimensional system:

$$
\xi(\infty)=\lim _{L_{t} \rightarrow \infty} \xi\left(L_{t}\right)
$$

On the other hand, $\xi\left(L_{t}\right)$ will grow forever, if the system floats to the metallic (delocalized) phase.

At the critical point, however, we expect the system to be scale invariant (diverging correlation length), i.e. $\xi\left(L_{t}\right)$ should scale exactly as $L_{t}$ as soon as finite size effects have died out. So one tries

$$
\Lambda\left(L_{t}\right):=\xi\left(L_{t}\right) / L_{t}
$$

as a scaling variable.

A further reason for this choice comes from the interpretation that, in the metallic regime, an appropriate conductance for the corresponding cubic system is given by Eq. (257) where $g_{\text {qua }}\left(L ; L_{t}\right) \approx \xi\left(L_{t}\right) / L$ yielding

$$
g_{\text {cube }}\left(L=L_{t}\right) \approx \Lambda\left(L_{t}\right)
$$

In the localized regime, where $\xi\left(L_{t}\right)$ approaches $\xi(\infty), \Lambda\left(L_{t}\right)$ represents the inverse logarithm of the corresponding conductance

$$
-\ln g_{\text {cube }}\left(L=L_{t}\right) \approx \Lambda^{-1}\left(L_{t}\right)
$$

Now, one-parameter scaling means that away from the critical point (where $\Lambda\left(L_{t}\right) \equiv \Lambda^{*}$ is a constant) $\Lambda\left(L_{t}\right)$ does not depend on the correlation length $\xi_{c}$ (which can be identified with $\xi(\infty)$ in the localized phase) and $L_{t}$ separately, but only on their dimensionless ratio

$$
\Lambda\left(L_{t}\right)=f\left(\frac{\xi_{c}}{L_{t}}\right) .
$$


Here $f$ is a called the scaling function. In the presence of LD transitions, $f$ is a two-valued function. Close to a critical point (where linearizing of the corresponding $\beta$-function is appropriate) the scaling function is of the form (cf. Eq. (160))

$$
f(x)=\Lambda^{*} \pm A_{( \pm)} x^{-\frac{1}{\nu}}
$$

where the positive (negative) sign marks the delocalized (localized) branch of $f: \beta(\ln \Lambda)>0$ $(\beta(\ln \Lambda)<0)$.

The general procedure to extract the scaling function from a large number of calculated localization lengths $\xi\left(L_{t}\right)$ is to find a quantity $\xi_{c}$ independent of $L_{t}$ such that the entire data set $\xi\left(L_{t} ; \tau\right)$ (where $\tau$ is a system parameter related to e.g. the disorder or Fermi energy with critical value $\tau^{*}=0$ ) collapses onto a single curve. This curve then defines $f$. In practice, this can be done by plotting the $\ln \Lambda\left(L_{t} ; \tau\right)$ curves on transparencies and moving them around by hand until they fall on top of each other or by a least squares fitting procedure. A typical example for a scaling function is shown in Fig. 24. After determining $\Lambda^{*}$ one can determine the critical exponent $\nu$ from a fit to Eq. (265).

It may, however, turn out to be difficult to find the critical value $\Lambda^{*}$. For example, it can happen that the curves $\Lambda\left(L_{t} ; \tau\right)$, for different choices of $L_{t}$, do not intersect at one point $\tau=0$. This can be due to the fact that the system size is not large enough to reach the asymptotic scaling regime, i.e. it can be a finite size effect. Assume that, at the true critical point $\tau=\tau^{*}=0$, the scaling variable still shows a dependence on $L_{t}$ of the form

$$
\Lambda\left(L_{t} ; \tau=0\right)-\Lambda^{*}=B L_{t}^{-y_{\mathrm{irr}}}
$$

Here $y_{\text {irr }}>0$ is a critical exponent. More convenient is $B L_{t}^{-y_{\text {irr }}}=\tilde{B}\left(L_{t} / \xi_{\text {irr }}\right)^{-y_{\text {irr }}}$ with $\tilde{B}$ of order 1 and an explicit scale $\xi_{\text {irr }}$ called irrelevant length. Since $\xi_{\text {irr }}$ stays finite the correction to $\Lambda^{*}$ decreases when $L_{t}$ becomes larger than $\xi_{\text {irr }}$.

$$
\lim _{L_{t} \rightarrow \infty} \Lambda\left(L_{t} ; \tau=0\right)=\Lambda^{*}
$$

In this sense, the length $\xi_{\text {irr }}$ becomes irrelevant in the large $L_{t}$ limit. However, in a finite size calculation one can never be sure that microscopic scales are already much smaller than $L_{t}$ and one has to face the fact that corrections to the ideal scaling, Eq. (265), can occur. They can then be analyzed by means of irrelevant length scales, Eq. (267).

The best studied system is the quantum Hall system in 2D. Although no metallic phase exists, the position of the critical point on the energy scale is precisely known for certain models due to spectral symmetries. This fact and the two-dimensionality enables to get results for critical quantities with high precision (see e.g. 68,72). Also a 3D analog of the quantum Hall system has been studied recently [31]. Further results are known for 2D systems with spin-orbit scattering (see e.g. [48,51, 118]) and for a number of 3D systems (see e.g. [64, 62, 73]). 


\section{STATISTICS AT THE TRANSITION}

The finite size scaling method made it possible to analyze a self-averaging quantity serving as scaling variable in the sense of one-parameter scaling theory. It demonstrates not only that one-parameter scaling does work, but also allows for explicit calculations of the critical exponent of the correlation length. However, not very much information comes out of this for the statistical properties of non-self-averaging quantities like conductance and LDOS. At least, by Eqs. 262,263) one can expect that the scaling variable $\Lambda$ of the finite size scaling method is related to the typical conductance $g_{t}$. The latter can be identified as the mean value in the delocalized phase and as the geometric mean in the localized phase. Since, in the delocalized phase the geometric mean is very close to the mean (the distribution

is Gaussian with $\left.\sqrt{\left\langle(\delta g)^{2}\right\rangle} /\langle g\rangle \approx 1 /\langle g\rangle \ll 1\right)$ it is reasonable to expect that the geometric mean

$$
g_{t}:=\exp (\langle\ln g\rangle)
$$

can serve as a typical conductance which in turn is a scaling variable.

In this section we will firstly consider the statistics of critical eigenstates. After the findings of the previous section we already expect them to show the multifractal scaling property. We will discuss the implications of this for the LDOS. Several arguments will point out that the typical conductance will determine the distributions of local quantities. After a brief discussion of critical energy level statistics we collect results for the critical conductance distribution.

\section{A. Multifractality Of Critical Eigenstates}

In finite size systems the correlation length $\xi_{c}$ of the electronic states is larger than the system size $L$ for a certain parameter range, $\Delta \tau$, around the critical parameter value $\tau=0$. These states are called critical states. In the thermodynamic limit $\Delta \tau \propto L^{-1 / \nu}$ where $\nu$ is the critical exponent of $\xi_{c}$.

After the pioneering works by Wegner [133 and Aoki [11] it became clear that the critical wave functions have a multifractal structure (for a review see [71] and references therein). The entire distribution of local amplitudes and its scaling behavior is encoded in the multifractal $f(\alpha)$ spectrum, as outlined in Sect. VIIq. The distribution $\mathcal{P}\left(P ; L_{b} / L\right)$ is broad on all length scales and close to a log-normal distribution. The most important quantity is the maximum position, $\alpha_{0}$, of $f(\alpha)$. It describes the scaling behavior of the geometric mean of what serves as a typical amplitude of a critical wave function.

In early works Aoki [11] gave a nice argument for the multifractal behavior of critical wave functions (although at that time the phrase multifractality was not yet common). His argument goes as follows. Consider the inverse participation number defined by 


$$
\mathcal{P}=\int_{\Omega} d^{d} r|\psi(\mathbf{r})|^{4}
$$

where $\Omega$ denotes a $d$-dimensional region with linear size $L$. If the wave function $\psi(\mathbf{r})$ is uniformly distributed - like in the delocalized phase - then $\mathcal{P} \propto L^{-d}$ and the participation ratio $p=\left(\mathcal{P} L^{d}\right)^{-1}$ is constant. In the localized regime $\mathcal{P} \approx \xi^{-d}$ and $p$ vanishes in the thermodynamic limit. At the transition point where the wave function is extended the participation ratio still has to vanish in the thermodynamic limit if the LD phenomenon is similar to a second order phase transition for which the participation ratio acts as an order parameter. Consequently, $\mathcal{P}$ scales with a power $d^{*}<d$. Wegner had already calculated the whole spectrum of exponents for generalized inverse participation numbers within the non-linear sigma-model [133]. This spectrum was interpreted as a multifractal spectrum by Castellani and Peliti [24]. After extensive numerical work (e.g. [109,117,66, 118]) the following description of the statistics of critical wave functions $\psi(\mathbf{r})$ is now established:

At the LD transition the distribution function $\mathcal{P}\left(P ; L_{b} / L\right)$ gives rise to the power law scaling for the moments,

$$
\left\langle\left[P\left(L_{b}\right)\right]^{q}\right\rangle_{L} \propto\left(L_{b} / L\right)^{d+\tau(q)},
$$

where $d+\tau(q)$ is a non-linear function of $q$. This non-linearity is a direct consequence of Aoki's observation that $d+\tau(2)=d+d^{*} \neq d+d$. In practice it turns out that, to a good accuracy, the disorder average can often be substituted by the spatial average over one wave function for a given configuration. Within numerical accuracy the resulting spectra are identical.

The corresponding (universal) distribution function can be described in terms of the single-humped, positive $f(\alpha)$ spectrum,

$$
\mathcal{P}\left(P ; L_{b} / L\right) d P \propto\left(L_{b} / L\right)^{d-f(\alpha)} d \alpha
$$

where $\alpha:=\ln P / \ln \left(L_{b} / L\right)$; As shown in Sect. $\mathrm{VIIC} f(\alpha)$ is related to $\tau(q)$ by a Legendre transformation

$$
f(\alpha(q))=\alpha(q) q-\tau(q), \quad \alpha(q)=d \tau(q) / d q
$$

The parabolic approximation (PA), Eq. (201), contains $\alpha_{0}$ as the only parameter besides $d$. This is due to the assumed validity of the PA at least up to $|q| \leq 1$. Equation (201) corresponds to a log-normal distribution centered around the typical value $P_{\mathrm{t}}=\exp <\ln P>\propto\left(L_{b} / L\right)^{\alpha_{0}}$ with log-variance proportional to $\alpha_{0}-d$. A simple oneparameter approximation for $f(\alpha)$ which takes into account that the support $[\alpha(\infty), \alpha(-\infty)]$ of $f(\alpha)$ is finite, is the semi-elliptic approximation (SEA)

$$
f(\alpha) \approx d \sqrt{1-\frac{\left(\alpha-\alpha_{0}\right)^{2}}{\alpha_{0}^{2}-d^{2}}} .
$$


To demonstrate that the distribution of local amplitudes of critical eigenstates is encoded in $f(\alpha)$ we discuss numerical results for a quantum Hall system (QHS) [110]. The quantum Hall system is the most convenient to study critical eigenstates since one knows the critical point exactly and it is only two-dimensional which keeps the numerical effort low.

The wave functions are calculated for the model of independent (spin-less) electrons subject to strong magnetic field and disorder. The disorder was implemented by a set of $\delta$-impurities with random positions and random strengths symmetric around zero. The microscopic length scale of the problem is the magnetic length $l_{B}$ defined by the size of an area penetrated by a single flux-quantum, $2 \pi l_{B}^{2}$. The representing Hamiltonian matrix was worked out in the Landau representation diagonalizing the clean system (no disorder). The clean case has the famous Landau level spectrum of highly degenerate and equally spaced levels at discrete energy values. The spacing is given by the cyclotron frequency $\hbar \omega_{c}=\hbar e B / m$. Disorder (symmetric around zero energy) broadens the Landau levels to Landau bands of non-degenerate states. For strong magnetic fields the broadened Landau bands are still separeted, the band-width $\Gamma$ being smaller than $\hbar \omega_{c}$ (see Fig. 25).

Restricting to one Landau band (it is most convenient to take the lowest) the LD transition takes place precisely at the center of the symmetric Landau band (cf. [72], 668]). A system of area $L^{2}$ is represented by a finite matrix of dimension $N=L^{2} /\left(2 \pi l_{B}^{2}\right)$. The numerical diagonalization yields the eigenvalues and eigenstates for any desired energy window within the lowest Landau band.

In Fig. 26 the squared amplitudes of a wave function from the center of the Landau band are shown together with the $f(\alpha)$ spectrum calculated from these amplitudes. The corresponding histogram of the logarithm of amplitudes (measured on a box of size $4 l_{B}^{2}$ ) is displayed in Fig. 27 together with the distribution function calculated from the $f(\alpha)$ spectrum using Eq. (271). These figures demonstrate that the distribution of amplitudes is (i) encoded in the $f(\alpha)$ spectrum and (ii) is close to a log-normal distribution characterized by one critical exponent $\alpha_{0}=2.28 \pm 0.03$ (the average over 130 critical states).

Figures 26, 27 correspond to a model of $\delta$-impurities resulting in a short range potential correlation. Similar calculations for a long range potential correlation (the potential correlation length is much larger than the magnetic length) have been performed within the Chalker-Coddington network model [74]. A corresponding critical wave function and its histogram are shown in Figs. 28, 29. They demonstrate the universality of the multifractal properties with respect to the potential correlation length.

To study the spatial correlations of amplitudes for a fixed energy consider the $q$-dependent correlations

$$
M^{[q]}\left(r, L_{b}, L\right):=\left\langle\left[P_{i}\left(L_{b}\right)\right]^{q}\left[P_{i+s}\left(L_{b}\right)\right]^{q}\right\rangle_{L}
$$

where the average is to be taken over all pairs of boxes with fixed distance $r=s L_{b}$.

For critical states where the microscopic scale ( $l_{B}$ in our case) and the macroscopic scale 
(the localization length $\xi$ in our case) are separated, one can expect power law behavior of $M^{[q]}$ in the regime

$$
l_{B} \ll L_{b}, r, L \ll \xi .
$$

Usually, in critical phenomena one studies correlations for infinite system size (and $L_{b}$ being microscopic) as a function of $r$ alone. This is justified if, for large enough system sizes $L$, the correlation function is independent of $L$ (for simplicity we neglect any trivial $L$ dependence due to pre-factors in the definition of the observable $P$ ). However, this is not true in the multifractal case. Multifractality reflects broadness of the distribution function $\mathcal{P}\left(P, L_{b} / L\right)$ on all length scales. The local box observable $P_{i}\left(L_{b}\right)$ depends on a large number of conditions for the entire system of linear size $L$, simultaneously. In the context of the LD transition coherence at zero temperature is due to quantum mechanical phase coherence of the electron's wave function, and disorder introduces a huge number of parameters, e.g. the position of point-scatterers. In the multifractal scaling case one has to face the fact that $M^{[q]}$ depends non-trivially on $L$, even for $L \rightarrow \infty$. We have incorporated such behavior already in our general discussion on correlation functions in Sect. VIIA.

Therefore, we consider the regime $l_{B} \ll L_{b}<r<L \ll \xi_{c}$ and make the ansatz

$$
M^{[q]}\left(r, L_{b}, L\right) \propto L_{b}^{x_{2}(q)} L^{-y_{2}(q)} r^{-z(q)} .
$$

The task is now to find the scaling relations between the set of exponents $x_{2}(q), y_{2}(q), z(q)$ and the $\tau(q)$ function. These scaling relations can be derived by requiring consistency with the limiting situations (i) where $r$ is of the order of $L_{b}$ and (ii) where $r$ is of the order of $L$. We find [25, [1]

$$
\begin{aligned}
y_{2}(q) & =d+\tau(2 q) \\
x_{2}(q) & =2 d+2 \tau(q) \\
z(q) & =d+2 \tau(q)-\tau(2 q) .
\end{aligned}
$$

It is worth mentioning that the sum $x_{2}(q)-y_{2}(q)-z(q)$ vanishes due to the normalization of the wave function.

The analytic behavior of $z(q)$ according to Eq. (279) is shown in Fig. 30. In general, it is non-negative and asymptotically bounded by the dimension $d$. To check on the validity of Eq. (279) Pracz et al. took 100 critical states of a system with $L=200 l_{B}$ and calculated $M^{[q]}\left(L_{b}, r, L\right)$ with fixed values $L_{b}=l_{B}, 4 l_{B} ; L=200 l_{B}$. Within the errors the validity of the scaling relations in Eqs. (279, 278) could be confirmed.

To include aspects of local energy statistics as well, let us consider the $q$-dependent correlation of box probabilities corresponding to two different eigenstates with energies $E$ and $E+\omega$

$$
M_{\omega}^{[q]}\left(r, L_{b}, L\right):=\left\langle\left[P_{i}\left(E ; L_{b}\right)\right]^{q}\left[P_{i+s}\left(E+\omega ; L_{b}\right)\right]^{q}\right\rangle_{L} .
$$


To understand the correlation behavior of non-localized states with respect to the energy separation one has to compare the relevant energy scales of the problem. These are the average level spacing $\Delta$ and the (frequency dependent) Thouless energy energy $E_{\mathrm{Th}}(\omega)$ corresponding to the time a wave packet (formed from states within an energy window of width $\omega)$ needs to diffuse through the system, $L^{2}=\left(\hbar / E_{\mathrm{Th}}(\omega)\right) D(\omega)$. Here $D(\omega)$ is the corresponding diffusion constant. According to Chalker [28], these scales give rise to the definition of two length scales depending on the energy separation $\omega$ :

$$
\begin{aligned}
& \tilde{L}_{\omega}:=\left(\omega / E_{\mathrm{Th}}(\omega)\right)^{-1 / 2} L \\
& L_{\omega}:=(\omega / \Delta)^{-1 / d} L .
\end{aligned}
$$

The first length scale, $\tilde{L}_{\omega}$, is the typical distance a wave packet will travel diffusively in a time $\hbar / \omega$. From this it is natural to assume that correlations between $P_{i}\left(E ; L_{b}\right)$ and $P_{i+s}\left(E+\omega ; L_{b}\right)$ will be present at least for distances $r \ll \tilde{L}_{\omega}$ whereas for larger distances the amplitudes are uncorrelated. Such uncorrelated behavior is typical for the standard random matrix theory approach to extended states in random systems. Recall, that in this theory it is assumed that the unitary matrices that diagonalize the Hamiltonian are distributed uniformly in the unitary group and no correlations (apart from the unitarity property) between different matrix elements occur. Thus, the presence of correlations here is an explicit breakdown of the no-preferential basis assumption. In electron systems with spatial disorder a preference to some basis is always given. This preference cannot be seen in $M_{\omega}^{[q]}$ for distances $r \gg \tilde{L}_{\omega}$.

The second length scale, $L_{\omega}$, is the linear size of a system with level spacing $\omega$. Two wave functions with energetic separation smaller than the level spacing show a spatial correlation behavior of its amplitudes similar to that corresponding to one of those wave functions, i.e. they are statistically indistinguishable.

At the critical point of the LD transition the typical conductance becomes a size independent quantity, $g^{*}$, and with the help of the Einstein relation between conductivity and diffusion one finds $\tilde{L}_{\omega}=\left(g^{*}\right)^{1 / d} L_{\omega}$ [28]. Since $g^{*}$ is of $\mathcal{O}(1)$ the two length scales coincide at the LD transition. Therefore, one can focus on the role of $L_{\omega}$.

Asking for the scaling properties of $M_{\omega}^{[q]}$ in the regime $L_{b}<r<L_{\omega} \leq L$ we make the ansatz (cf. Eq. (276))

$$
M_{\omega}^{[q]} \propto L_{b}^{X_{2}(q)} r^{-z(q)} L_{\omega}^{Z(q)} L^{-Y_{2}(q)} .
$$

Here we have already anticipated that the exponent with respect to $r$ is $z(q)$, as given before. As in the case of $M^{[q]}$ for a fixed energy by considering limiting situations one finds

$$
\begin{aligned}
X_{2}(q) & =2 d+2 \tau(q)=Y_{2}(q) \\
z(q)=Z(q) & =d+2 \tau(q)-\tau(2 q) .
\end{aligned}
$$


Now the following conclusions can be drawn: 1 . The result for $z(q)$ is the same as in the case of zero energy separation. 2. The energy separation $\omega$ is not an independent scaling parameter but appears only in the combination $L_{\omega} / r$. 3. The exponent corresponding to the box size, $x_{2}(q)=X_{2}(q)$, is not affected by a finite energy separation but the exponent corresponding to the system size $L$ (which is $y_{2}(q)$ for zero energy separation) splits up into the exponents $Z(q)$ (corresponding to $L_{\omega}$ ) and $Y_{2}(q)$ (corresponding to $L$ for finite energy separation).

In the work by Pracz et al. it was demonstrated that the scaling relations are consistent with numerical results. Similar findings have been obtained by Metzler [96 within the scattering matrix network model (Chalker Coddington model).

\section{B. Local Density Of States As Order Parameter}

Having established the role of $r / L_{\omega}$ as the relevant scaling parameter for correlations of eigenstate amplitudes with universal exponent $z(q)$ (related to $\tau(q)$ by a scaling relation) let us now discuss the consequences of this for the interpretation of the local density of states being an order parameter of the LD transition. With the smearing-out of the $\delta$-functions the LDOS is given by (see Eq. (49))

$$
\rho(E, \mathbf{r})=\Gamma(E)^{-1}|\psi(E, \mathbf{r})|^{2}
$$

where $|\psi(E, \mathbf{r})|^{2}$ stands for the microcanonical average of squared amplitudes at a given energy $E$. Since $\Gamma(E)$ behaves as $L^{-d}$, the scaling behavior of the LDOS is determined by that of the wave function. Consequently, we have

$$
\left\langle[\rho(E, \mathbf{r})]^{q}\right\rangle_{L} \propto L^{(q-1) d-\tau(q)}
$$

and for the typical value

$$
\rho_{\mathrm{t}}=\exp \left[\langle\ln (\rho(\mathbf{r}))\rangle_{L}\right] \propto L^{d-\alpha_{0}}
$$

which does reflect the LD transition. Scaling $L$ with the correlation length $\xi_{c} \propto \tau^{-\nu}$ the typical LDOS vanishes on approaching the critical point with exponent $\beta_{\mathrm{t}}=\nu\left(\alpha_{0}-d\right)(\approx 0.7$ in quantum Hall systems).

The behavior of the typical LDOS is, according to the argument presented in the beginning of this section, similar to that of the participation ratio introduced by Wegner 133. The participation ratio was based on the second moment of local probability. Higher moments will work as well and our notion of typical LDOS is just a bit more convenient since it focuses on a typical value rather than on some specific moments.

Our interpretation is also consistent with the findings for the LDOS in the localized and delocalized phase as discussed in Sect. V1. Therefore, we summarize the findings: 
1. In the localized phase the typical local density of states vanishes.

2. In the delocalized phase the typical local density of states is finite and positive.

3. Approaching the LD transition from the delocalized phase the typical LDOS vanishes with a positive critical exponent,

$$
\beta_{\mathrm{t}}=\nu\left(\alpha_{0}-d\right)
$$

A schematic picture for the typical LDOS that stresses its role as order parameter is shown in Fig. 31.

The unconventional feature as compared to ordinary critical phenomena lies in the facts that

1. The order parameter field has a broad distribution resulting in a non-linear dependence of exponents on the degree of moments considered (multifractality).

2. The average value shows a vanishing scaling exponent while the typical value gives rise to a positive scaling exponent

The scaling relations that we derived for the wave functions amplitudes transform to scaling relations of the LDOS since each box amplitude has to be multiplied by a constant factor of $L^{d}$,

$$
\left\langle\left(\rho\left(E, \mathbf{r}_{1}\right)\right)^{q}\left(\rho\left(E+\omega, \mathbf{r}_{2}\right)\right)^{q}\right\rangle_{L} \propto\left(r / L_{\omega}\right)^{-z(q)} L^{-\tilde{z}(q)}
$$

with

$$
z(q)=d+2 \tau(q)-\tau(2 q), \quad \tilde{z}(q)=2(1-q) d+2 \tau(q)
$$

These scaling relations form, in the sense of Sect. VIIA, the appropriate scaling relations connecting the spatial correlations of the local order parameter field to its scaling dimensions (cf. Eqs. (287), 290), (291)).

We mention that $\eta:=z(1)=d-D(2) \approx 0.4 \neq \tilde{\eta}:=\tilde{z}(1)=0$ (for the correlator of the density of states in quantum Hall systems) with $L_{\omega} / r$ forming the scaling parameter is equivalent (cf. [28,67]) to the phenomenon of anomalous diffusion found by Chalker and Daniell [27]. As pointed out in [27], the anomalous character of diffusion lies in the nonGaussian dispersion of a wave packet in time $t$ despite the fact that the average diameter grows like $\sqrt{t}$. This non-Gaussian time dispersion is caused by the multifractal character of eigenstates.

A further support for the order parameter interpretation comes from conformal mapping ideas for $2 \mathrm{D}$ systems. In ordinary critical phenomena theory, the following assumption for critical correlation functions $\chi(r) \propto r^{-\tilde{\eta}}$ seems plausible: Scale invariance, reflected by power 
laws, should hold also for local scale transformations which preserve angles but may change scales locally (called conformal mappings) [22].

Thus, the main idea behind conformal mapping arguments in scaling theory is an extension of a homogeneity law for correlation functions with respect to rescaling. Such a law exists also in the multifractal case, since any rescaling of all length scales in the LDOS correlator (for a fixed energy) by the same scaling factor $s$ leads to

$$
\left\langle\rho^{q}(s \mathbf{r}) \rho^{q}\left(s \mathbf{r}^{\prime}\right)\right\rangle_{s L}=s^{-\tilde{z}(q)}\left\langle\rho^{q}(\mathbf{r}) \rho^{q}\left(\mathbf{r}^{\prime}\right)\right\rangle_{L} .
$$

where $\tilde{z}(q)$ is given by Eq. (291).

Extending this law to conformal mappings of a geometry $\Omega$ to geometry $\tilde{\Omega}$ leads, for large but finite 2D systems, to

$$
\frac{\left\langle\rho^{q}\left(w\left(z_{1}\right)\right) \rho^{q}\left(w\left(z_{2}\right)\right)\right\rangle_{\tilde{\Omega}}}{\left\langle\rho^{q}\left(z_{1}\right) \rho^{q}\left(z_{2}\right)\right\rangle_{\Omega}}=\left|w^{\prime}\left(z_{1}\right)\right|^{-\frac{\tilde{z}(q)}{2}}\left|w^{\prime}\left(z_{2}\right)\right|^{\mid \frac{\tilde{z}(q)}{2}},
$$

where $w(z)$ is any holomorphic function of complex coordinate $z$ and $w^{\prime}(z)$ denotes the derivative.

By choosing $w(z)=\left(L_{T} / 2 \pi\right) \ln z$ which maps the plane onto a strip one can show (cf. [22]) that the correlator in the strip is characterized by a $q$-dependent quasi-1D localization length $\xi\left(q ; L_{t}\right)$ which is related to $\tilde{z}(q)$ by

$$
\xi\left(q ; L_{t}\right) / L_{t}=2 /(\pi \tilde{z}(q)) .
$$

In FSS calculations it is rather the average of the logarithm of the correlation function which is calculated by knowing that this quantity defines a typical localization length. Therefore, the result of Eq. (294) leads in particular (consider $q$ close to 0) to the relation

$$
\Lambda^{*}=\frac{2}{\pi\left(\alpha_{0}-d\right)}, \quad d=2 .
$$

Here $\Lambda^{*}$ appears since it is just the typical localization length at criticality divided by the width $L_{t}$. Equation (294) has been confirmed in numerical calculations by Dohmen et al. [40], and Eq. (295) is, so far, in accordance with all known numerical results.

\section{Conductance As Scaling Variable}

The fact that the critical value $\alpha_{0}-d$ is related to the critical value of the scaling variable (at least in 2D) is an important hint for the typical conductance $g_{\mathrm{t}}$ being a one-parameter scaling variable. The reasoning behind this statement goes as follows: as pointed out in Sect. VIII the scaling variable $\Lambda\left(L_{t}\right)$ of finite size scaling in quasi-1D can be related to the typical conductance of a cube $g_{\mathrm{t}}\left(L=L_{t}\right)$. From the $2+\epsilon$ field theoretic calculations 
at the LD transition [133] as well as from the multifractal LDOS found in 2D [50] we can conclude: if the correlation length is larger than system size and the typical conductance is still large, the eigenstates behave critical and are multifractal. The multifractal exponent $\alpha_{0}-d$ is determined by $g_{\mathrm{t}}$ and

$$
\alpha_{0}-d \propto 1 / g_{t}
$$

At a generic critical point with $\Lambda^{*} \sim g_{t} \sim \mathcal{O}(1)$ the conformal mapping relation tells, that also here the value of $\alpha_{0}-d$ is determined by $g_{t}$. This means that the bulk of the LDOS distribution function is, in the critical regime, already determined by the value of typical conductance.

Before we collect results for the conductance distribution at criticality we discuss a similar object related to the level statistics. Not only the LDOS and conductance distributions should be universal functions at criticality (see Eq. (181)), but also e.g. the level spacing distribution $P(s ; L)$ and the two-level correlation function $R(s ; L)$. To study these quantities in view of the one-parameter scaling picture was put forward in a work by Shklovskii et al. [119. These authors pointed out that the study of the level spacing distribution $P(s ; L)$ is, starting from a Hamiltonian modeling, numerically much easier to perform than calculating conductances. Only the spectrum has to be calculated. According to the one-parameter scaling picture $P(s ; L)$ should obey the following scaling behavior

$$
\lim _{L \rightarrow \infty} P(s ; L)=\lim _{L \rightarrow \infty} F\left(s ; \alpha_{L}\right)=P_{\mathrm{L}, \mathrm{D}, \mathrm{C}}(s) .
$$

Here $F\left(s ; \alpha_{L}\right)$ is a function that does no longer depend on microscopic details, but only on one scale dependent parameter $\alpha_{L}$. Depending on the initial value of $\alpha_{L}$ the system flows to either the localized fixed point $(\mathrm{L})$, the delocalized fixed point $(\mathrm{D})$ or it stays at the critical fixed point $(\mathrm{C})$. In terms of the typical conductance, $\alpha_{L}=g_{\mathrm{t}}(L)$, the fixed points are characterized by

$$
\lim _{L \rightarrow \infty} g_{\mathrm{t}}(L)=\left\{\begin{array}{cc}
0 & (\mathrm{~L}) \\
g_{\mathrm{t}}^{*} & (\mathrm{C}) \\
\infty & (\mathrm{D})
\end{array}\right\} .
$$

The corresponding asymptotic level-spacing distributions $P_{\mathrm{L}, \mathrm{D}, \mathrm{C}}(s)$ are given by the Wigner surmise, Eq. (137),

$$
P_{\mathrm{D}}(s)=A_{\beta} s^{\beta} \exp \left(-B_{\beta} s^{2}\right)
$$

the Poisson distribution, Eq. (120),

$$
P_{\mathrm{L}}(s)=\exp (-s),
$$


and by a, yet unknown, critical distribution $P_{\mathrm{C}}(s)$.

Numerical studies by several authors (e.g. [119], [49], [65], [135], [54]) have demonstrated that a size independent critical distribution $P_{\mathrm{C}}(s)$ distribution exists, once the parameter $\tau$ triggering the LD transition is put to its critical value $\tau=0$. It is also known that, qualitatively, the distribution resembles the Wigner surmise for values $s \leq s_{0} \approx 2$ and the Poisson law for $s \geq s_{0}$. At $s_{0}$ the distributions intersect. Furthermore, the way the critical distribution is approached allows for a calculation of the critical exponent of the correlation length $\nu$. This can be done in several ways. The authors of 119 took as a scaling variable the integral over $P(s ; L)$ up to $s_{0}$ (one could use also some other point)

$$
\alpha(L ; \tau):=\int_{0}^{s_{0}} d s P(s ; L ; \tau) .
$$

and analyzed it according to the general ansatz, (Eq. (160),

$$
\alpha(L ; \tau)=\alpha^{*}+A \tau L^{1 / \nu}+\mathcal{O}\left(\tau^{2}\right) .
$$

The values for $\nu$ found by this method do agree with those found in FSS analysis within the errors.

As to the precise form of $P_{\mathrm{C}}(s)$ there exists no conclusive theory, except that the very small $s$ behavior is again dictated by the level repulsion $\propto s^{\beta}$. For example, the tail of $P_{\mathrm{C}}(s)$ is not exactly known. It seems now to be established that there is a leading exponential tail with sub-leading stretched exponential tail contributions. The question about the form of the tail is related [6] to the question about the relation between the number variance $\Sigma^{2}$ and the average level number $\langle n\rangle$, (see Sect. VI). Based on one-parameter scaling arguments Aronov et al. 114] had predicted (for the LD transition point without magnetic fields) the following scaling law, for large $\langle n\rangle$,

$$
\Sigma^{2} \propto(\langle n\rangle)^{1-\frac{1}{d \nu}} .
$$

However, in this work the anomalous diffusion at criticality (or in other words the multifractality) was not taken into account. Correcting this omission leads to an additional linear term, characterized by a finite level compressibility [33]

$$
\frac{d \Sigma^{2}}{d\langle n\rangle}=\frac{d-D(2)}{2 d} \text {. }
$$

In view of the finite level compressibility Eq. (303) describes sub-leading contributions to $\Sigma^{2}$.

It is very interesting that the analysis of $\Sigma^{2}$ as a function of $\langle n\rangle$ seems to allow for computing the correlation length exponent $\nu$ and the fractal dimension $D(2)$ related to the order parameter exponent $\beta_{\mathrm{t}}=\left(\alpha_{0}-d\right) \nu$ (within the parabolic approximation to $f(\alpha)$ $\left.D(2) \approx d-2\left(\alpha_{0}-d\right)\right)$. 
As to the critical conductance distribution the general considerations formulated in Eqs. (297,298) for the level spacing distribution do apply as well. The precise form of the critical conductance distribution $\mathcal{P}^{*}(g)$ is not known. Based on the $2+\epsilon$ calculations by Al'tshuler et al. [5], Shapiro and coworkers [36] predicted that power law tails should occur. So far, this could not neither be proved nor disproved by numerical means. However, in a work by Markos and Kramer [91] it was shown that the critical conductance distribution is universal for a 3D system without magnetic field, i.e. it does not depend on system size $L$ and does not depend on which parameter $\tau$ is taken to its critical point value. The distribution showed fluctuations $\sqrt{\left\langle(\delta g)^{2}\right\rangle}$ that are of the same order $(\mathcal{O}(1))$ as the mean value of the distribution. The precise form of the tails could not be determined.

The scaling of the conductance distribution close to criticality follows from expanding the scaling variable around criticality which brings the exponent $\nu$ into play,

$$
\mathcal{P}(g ; L ; \tau)=F\left(g ; \alpha_{L}(\tau)\right)=\mathcal{P}^{*}(g)+F^{\prime}\left(g ; \alpha^{*}\right) A \tau L^{1 / \nu}+\ldots,
$$

where $F^{\prime}$ denotes the derivative of the function $F$ with respect to the scaling variable $\alpha_{L}$. To analyze the behavior of $\mathcal{P}(g ; L ; \tau)$ at one particular point is numerically not advantageous. Therefore some integrated quantities work better. However, as explained earlier, the chosen quantity should not be sensible to the tails of the distribution. Therefore, one should refrain from taking moments. Unfortunately, not much has been done in this direction.

Fastenrath et al. [53] have investigated a quantity which is similar to the dissipative conductance, the so-called Thouless number, for quantum Hall systems and studied the distribution function. Also in this case it turned out that, at the critical point, the distribution function was scale independent with fluctuations of the same order as the typical value. They explicitly took the typical Thouless number $g_{\mathrm{t}}=\exp \langle\ln g\rangle$ and analyzed it according to

$$
g_{\mathrm{t}}(L ; \tau)=g_{\mathrm{t}}^{*}+A \tau L^{1 / \nu}+\ldots .
$$

They found a value for $\nu(\approx 2.3)$ which is in good agreement with the value obtained by FSS. Thus, their observation gives further support that the typical conductance is an appropriate scaling variable.

Cho and Fisher [34] have performed a calculation of a true two-probe conductance within the Chalker-Coddington network model for the quantum Hall effect. There findings are consistent with the two-probe experiment by Cobden and Kogan 35 mentioned in the introduction: the distribution is almost uniform between 0 and 1 . This means in particular that the fluctuations are of the same order as the average value. For this uniform distribution on a finite interval the average value is already a convenient choice for the scaling variable and Cho and Fisher were able to show that the average value shows scaling of the form written in Eq. (306) with the correct critical exponent $\nu \approx 2.3$. Similar results were also obtained by Wang et al. [128]. 
Thus, although the precise form of critical conductance distributions is not known, there are a number of results which confirm that it is universal and that the notion of typical conductance allows for studying the critical exponent of the correlation length.

\section{SUMMARY}

In this review we have discussed a few fundamental experiments that show the importance of quantum mechanical interference effects for transport measurements in mesoscopic systems. Static disorder leads to complicated interference patterns of electronic wave functions which can cause large fluctuations in physical quantities. In addition, they can lead to localization of states to a finite volume within the mesoscopic system. Mesoscopic systems can be experimentally realized at very low temperatures $(\leq 1 \mathrm{~K})$ and with small devices $(\leq 1 \mu \mathrm{m})$ such that the phase coherence length $L_{\phi}$ is larger than system sizes $L$.

We introduced the basic physical quantities which are the local and global density of states (LDOS and DOS), the global conductance which is (in atomic units $e^{2} / h$ ) given by a dimensionless quantity $g$, and the conductivity tensor $\sigma_{\mu \nu}\left(\mathbf{r}, \mathbf{r}^{\prime} ; \omega\right.$ ) (or equivalently the diffusion function $D_{\mu \nu}\left(\mathbf{r}, \mathbf{r}^{\prime} ; \omega\right)$ ), related to local charge transport. The relevant physical energy scales are the quantum kinematic scales of level spacing $\Delta$ and Fermi energy $E_{\mathrm{F}}$, and the transport energy scale set by the Thouless energy $E_{\mathrm{Th}}$. The conductance is given by the Thouless formula, $g=E_{\mathrm{Th}} / \Delta$. A quantum kinematic length scales is the Fermi wavelength $\lambda_{F}$, and transport length scales are the microscopic mean free path $l_{e}$ and the localization length $\xi$ for localized states.

We discussed models for disordered mesoscopic electron systems in terms of Hamiltonians and scattering $S$-matrices. From the Hamiltonian models energy eigenvalues $\varepsilon_{\alpha}$ and eigenvectors $\psi_{\alpha}$ can be calculated. Their statistical properties determine the statistics of the most important quantity, the Green's function which determines all of the relevant quantities and scales. Within the $S$-matrix modeling the transmission strengths $T_{\alpha \beta}$ are the most important quantities. They directly determine the conductance via the Büttiker formula, $g=\sum_{\alpha \beta} T_{\alpha \beta}$. In addition, certain network $S$-matrix models also allow for a determination of energy eigenvalues and eigenvectors.

For the statistical and scaling properties of mesoscopic electron systems we collected the following insights.

1. Ideal localized systems (with vanishing localization length $\xi$, i.e. vanishing conductance $g=0$ ) are characterized by a compressible spectrum of uncorrelated energies and a LDOS that typically vanishes, although the average DOS is finite.

2. Ideal delocalized systems (with infinite Thouless energy $E_{\mathrm{Th}}$, i.e. infinite conductance $g=\infty)$ are characterized by an incompressible spectrum of correlated energies and 
Gaussian distributed wavefunctions which leads to an exponential tail for the LDOS probability distribution.

3. Changing control parameters, $\tau$, of the mesoscopic system like particle density, disorder strength, pressure or applied fields can drive the system through a disorder induced localization-delocalization (LD) transition (at $\tau=0$ ) which resembles a critical phenomenon. Due to the randomness of disorder the transition has to be described in terms of distribution functions the flow of which with respect to system size (scaling) forms the object of interest in a critical phenomenon description.

4. A one-parameter scaling theory for distribution functions of global quantities like conductance and energy-level spacing seems to work well. It states that the distributions are essentially determined by one scale dependent parameter $\alpha_{L}$, called scaling variable, that determines the flow, $\mathcal{P}(X ; L) \approx F\left(X ; \alpha_{L}\right)$.

5. A possible scaling variable for the LD transition is given by the geometric mean of the conductance distribution which we called typical conductance $g_{\mathrm{t}}$. With increasing system size $g_{\mathrm{t}}$ can approach the idealized localized phase $\left(g_{\mathrm{t}}=0\right)$, or it can approach the idealized delocalized phase $\left(g_{\mathrm{t}}=\infty\right)$. Corrections to distribution functions as compared to the ideal situations are controlled by finite values of $g_{\mathrm{t}}$. Right at the LD transition $g_{\mathrm{t}}$ will become a size independent universal quantity $g_{\mathrm{t}}^{*}$ that is of order 1 in generic LD transitions. The corresponding distribution functions of global quantities like conductance and level-spacing are universal scale-independent functions.

6. The fictitious system size $\xi_{c}$ for which $g_{\mathrm{t}}\left(\xi_{c}\right) \approx g_{\mathrm{t}}^{*}$ defines the correlation length of the LD transition. In the localized phase it can be identified with the localization length $\xi$. Close to the transition point, where it diverges, it scales with a critical exponent, $\xi_{c} \propto \tau^{-\nu}$.

7. While in the localized phase the typical (not the average) LDOS vanishes, the LDOS shows power law scaling in non-localized phases. The average value is always scaleindependent. In the ideal delocalized phase one exponent, simply related to dimensionality $d$, controls the scaling of the whole distribution function. At the LD transition a spectrum of scaling exponents is necessary to describe the scaling of the distribution which is close to a log-normal distribution. The spectrum has an interpretation in terms of a spectrum of fractal dimensions (multifractal spectrum). Still, there is one particular exponent, related to the typical value of the LDOS, which determines the log-normal approximation for the distribution. It is called $\alpha_{0}-d>0$ and is determined by the value of the scaling variable at criticality. Thus, multifractality is not in conflict with one-parameter scaling theory. 
8. On the contrary, the multifractal scaling of the LDOS opens the possibility to consider the typical value of the LDOS as an appropriate order parameter for the LD transition. The corresponding critical exponent is given by $\beta_{\mathrm{t}}=\nu\left(\alpha_{0}-d\right)$. Further support to this idea comes from the observation that a number of scaling relations for the LDOS can be formulated that are in close analogy to similar relations in ordinary critical phenomena.

In short, the occurrence of broad distributions and multifractality in disordered mesoscopic electron systems is by no means in contradiction to the one-parameter scaling theory, but points out that only typical rather than average quantities can serve as order parameter and scaling variable in this theory.

\section{Acknowledgments}

I gratefully acknowledge helpful discussions with A. Altland, Y. Avron, M. Backhaus, J. Chalker, A. Dohmen, P. Freche, J. Hajdu, B. Huckestein, R. Klesse, I. Lerner, M. Metzler, D. Polyakov, K. Pracz, B. Shapiro and M. Zirnbauer.

This work was supported by SFB 341 of the Deutsche Forschungsgemeinschaft and the MINERVA foundation. I thank the Department of Theoretical Physics in Oxford, U.K., and the Institute of Theoretical Physics at the Technion, Haifa, for the kind hospitality during visits in which part of the work was carried out.

\section{APPENDIX A: BRIEF ACCOUNT OF LINEAR RESPONSE THEORY}

We start with a general outline of how to calculate linear response quantities when the system is specified by a Hamiltonian. We then specialize to additive systems where the resolvent $G(z):=(z-H)^{-1}$ of the corresponding one-particle Hamiltonian (taken at complex energies $z$ ) yields all the basic physical quantities. Studying transport in multiprobe geometries will lead to the concept of the scattering matrix.

Consider a many-particle system characterized by the Hamilton operator $H$ coupled to a linear increment of a force field, $F$,

$$
\mathcal{H}_{F}=H-X F
$$

where $X$ is a hermitean operator of the system. The system is assumed to be in an equilibrium state at temperature $T$ described by the (grand) canonical density operator $\rho_{F}$. The thermodynamic susceptibility $\chi_{Y X}$ is defined as

$$
\chi_{Y X}:=\frac{\partial\langle Y\rangle}{\partial F} .
$$

Linearizing $\rho_{F}$ with respect to the increment $F$ the thermodynamic susceptibility reads 


$$
\chi_{Y X}=\beta\langle\Delta X ; \Delta Y\rangle
$$

where the bilinear expression (Kubo scalar product)

$$
\langle X ; Y\rangle:=\frac{1}{\beta} \int_{0}^{\beta} d \lambda\langle X Y(i \hbar \lambda)\rangle=\langle Y ; X\rangle^{*}
$$

is called the canonical correlator of $X$ and $Y$ with $\beta=\left(k_{B} T\right)^{-1}, \Delta X:=X-\langle X\rangle$, and $X(t)=$ $e^{(i / \hbar) H t} X e^{-(i / \hbar) H t}$ is the time evolution of $X$ in the Heisenberg picture. The isothermal susceptibility describes an idealized linear response situation where the force field does not disturb equilibrium, but is just a parameter field of the system.

We turn now to the dynamic linear response problem [83, 56] where the following (Kubo) process is considered: the increment in an applied force field $F(t)$ depends on time $t$ and is switched on adiabatically at $t=-\infty$. The total Hamilton operator is

$$
\mathcal{H}_{F}(t)=H-X F(t) e^{\eta t}
$$

where the slowness parameter $\eta$ has to be much smaller than the inverse of a typical positive time scale $t_{m}$ at which the response to the field is measured The system is assumed to be in equilibrium for $t=-\infty$ described by the density operator $\rho(F=0)$ and isolated for $t>-\infty$. Restricting our consideration to one Fourier component of the force field,

$$
F(t)=F e^{-i \omega t}
$$

the resulting linear increment in the Fourier component of the mean value of an observable $Y$ is

$$
\langle Y\rangle_{F}=\chi_{Y X}(z) F
$$

where the dynamic susceptibility $\chi_{Y X}(z)$ is given by the Kubo formula

$$
\chi_{Y X}(z)=\beta(\dot{X} \| Y)[z]=\beta(\Delta \dot{X} \| \Delta Y)[z]
$$

Here $\dot{X}$ denotes the time derivative of $X$ and the dynamic correlator

$$
(X \| Y)[z]:=\int_{0}^{\infty} d t e^{i z t}\langle X ; Y(t)\rangle
$$

appears as the Laplace transform $(z=\omega+i \eta)$ of the time dependent canonical correlator.

Integrating by parts in Eq. (A8) yields the fundamental equation of motion for dynamic correlators

$$
(\dot{X} \| Y)[z]=\langle X ; Y\rangle+i z(X \| Y)[z]=-(X \| \dot{Y})[z]
$$


Another important equation of motion, referred to as Kubo relation, holds for the canonical correlator

$$
\langle\dot{X} ; Y\rangle=\frac{-i}{\beta \hbar}\langle[X, Y]\rangle
$$

where $[X, Y]$ denotes the commutator of $X$ and $Y$. It is worth mentioning that the second term in Eq. (A10) can be finite in the case of zero frequencies, $\omega=0$, since it defines the long time average of $\langle X ; Y(t)\rangle$.

$$
\overline{\langle X ; Y\rangle}:=\lim _{\eta \rightarrow+0} \eta(X \| Y)[i \eta] .
$$

Equations (A3, A8, A10, A11) form the backbone of any linear response theory (cf. [83, 56]). With these we can now write down the expressions for the physical quantities considered in Sect. [II.

The local charge density, $q(\mathbf{r})$, can be related to local electric potentials, $U(\mathbf{r})$, by the charge response function, $\Pi\left(\mathbf{r}, \mathbf{r}^{\prime} ; \omega\right)$ 円,

$$
q(\mathbf{r})=\int d^{d} r^{\prime} \Pi\left(\mathbf{r}, \mathbf{r}^{\prime} ; \omega\right) U\left(\mathbf{r}^{\prime}\right)
$$

Charge response and conductivity (Eq. (10)) are related due to the continuity equation

$$
\begin{aligned}
\operatorname{div} \mathbf{j}(\mathbf{r})-i \omega q(\mathbf{r}) & =0 \\
\sum_{\mu \nu} \frac{\partial}{\partial r_{\mu}} \frac{\partial}{\partial r_{\nu}^{\prime}} \sigma_{\mu \nu}\left(\mathbf{r}, \mathbf{r}^{\prime} ; \omega\right) & =i \omega \Pi\left(\mathbf{r}, \mathbf{r}^{\prime} ; \omega\right)
\end{aligned}
$$

The particle density $n(\mathbf{r})$ couples to the local chemical potential $\mu(\mathbf{r})$ in the same way as the local charge $q(\mathbf{r})=-e n(\mathbf{r})$ couples to the local electric potential $U(\mathbf{r})$ and thus the thermodynamic charge response reads

$$
\Pi\left(\mathbf{r}, \mathbf{r}^{\prime}\right)=-\beta\left\langle\Delta q\left(\mathbf{r}^{\prime}\right) ; \Delta q(\mathbf{r})\right\rangle, \quad \int d^{d} r^{\prime} \Pi\left(\mathbf{r}, \mathbf{r}^{\prime}\right)=e^{2} \rho(\mathbf{r} ; \mu)
$$

while the dynamic charge response reads

$$
\Pi\left(\mathbf{r}, \mathbf{r}^{\prime} ; \omega\right)=-\beta\left(\Delta \dot{q}\left(\mathbf{r}^{\prime}\right) \| \Delta q(\mathbf{r})\right)[z]=\Pi\left(\mathbf{r}, \mathbf{r}^{\prime}\right)-i \beta z\left(\Delta q\left(\mathbf{r}^{\prime}\right) \| \Delta q(\mathbf{r})\right)[z]
$$

Note, that the equilibrium charge response $\Pi\left(\mathbf{r}, \mathbf{r}^{\prime}\right)$ is not equal to the $\omega \rightarrow 0$ limit of the dynamical charge response $\Pi\left(\mathbf{r}, \mathbf{r}^{\prime} ; \omega\right)$. This can be also seen when considering the response to homogeneous potentials. While a homogeneous electric potential does not create a charge response, and hence $\int d^{d} r^{\prime} \Pi\left(\mathbf{r}, \mathbf{r}^{\prime} ; \omega\right)=0$, shifting the overall chemical potential by a constant usually leads to a change in the particle density, i.e. $\int d^{d} r^{\prime} \Pi\left(\mathbf{r}, \mathbf{r}^{\prime}\right)=e^{2} \rho(\mathbf{r} ; \mu)$.

\footnotetext{
${ }^{9}$ For simplicity we drop the dependence on the time dependent vector potential.
} 
According to the general scheme described above, The conductivity tensor appears as the dynamic current correlator

$$
\sigma_{\mu \nu}\left(\mathbf{r}, \mathbf{r}^{\prime} ; \omega\right)=\beta\left(j_{\nu}\left(\mathbf{r}^{\prime}\right) \| j_{\mu}(\mathbf{r})\right)[z]
$$

and the relation in Eq. (A14) can be viewed as a special case of Eq. (A10). The corresponding global conductivity tensor reads in the thermodynamic limit $L \rightarrow \infty$

$$
\sigma_{\mu \nu}(\omega)=\frac{e^{2}}{L^{d}} \beta\left(v_{\nu} \| v_{\mu}\right)[z]
$$

where $\mathbf{v}$ denotes the velocity operator of electrons.

More delicate is the representation of the global conductance coefficients $G_{k m}(\omega)$, since one has to know the local potential distribution within the conductor as a functional of applied electro-chemical potentials $U_{m}$ and we do not go into the detail of this interesting problem. However, due to the continuity equation the situation simplifies for the d.c. situation: The current in any lead is independent of the actual potential distribution $U(\mathbf{r})$ within the conductor (cf. [70]) and $G_{k m}$ is determined by the dynamical correlator of asymptotic current operators which are the time derivatives of the total charge operator, $I_{k}=\dot{Q}_{k}$,

$$
G_{k m}=-\beta\left(\dot{Q}_{m} \| \dot{Q}_{k}\right)
$$

where omission of the argument $[z]$ refers to the d.c. situation.

Still, the calculation of the correlators requires the knowledge of the many particle density operator $\rho_{F=0}$ and the machinery of many-body physics is necessary to get quantitative results.

Assuming additive electron Hamilton operators like the ones introduced in Sect. $\mathbb{\nabla}$ the correlators can be written in closed form with the help of the one-particle resolvent (Green's function in real space representation)

$$
G^{ \pm}(E):=(E \pm i \epsilon-H)^{-1}, \quad \epsilon \rightarrow+0
$$

of the corresponding one-particle Hamiltonian $H$. It defines a spectral resolution via

$$
\delta(E-H)=(2 \pi i)^{-1}\left(G^{-}(E)-G^{+}(E)\right) .
$$

With the help of the Fermi distribution function

$$
f(E)=\left[1+e^{\beta(E-\mu)}\right]^{-1},
$$

(where $\mu$ denotes the chemical potential) average values can be calculated by

$$
\langle X\rangle=\frac{1}{2 \pi i} \int d E f(E) \operatorname{Tr}\left\{G^{-}(E) X-G^{+}(E) X\right\} .
$$

The canonical correlator reads 


$$
\langle\Delta X ; \Delta Y\rangle=\frac{i}{2 \pi \beta} \int d E f(E) \operatorname{Tr}\left\{X G^{-} Y G^{-}-X G^{+} Y G^{+}\right\}(E),
$$

and identifying $\eta$ with $2 \epsilon \hbar^{-1}$ yields

$$
(X \| Y)[z]=\frac{\hbar}{2 \pi \beta} \int d E f(E) \operatorname{Tr}\left\{\left(G^{-}-G^{+}\right)_{E}\left(X G_{E-\hbar \omega}^{-} G_{E}^{-} Y-Y G_{E+\hbar \omega}^{+} G_{E}^{+} X\right)\right\} .
$$

for the dynamical correlator, provided the equilibrium mean values of $X$ or $Y$ vanish.

Since the Fermi function is a step function at $T=0$ the general structure $\int d E f(E) A(E)$ occurring in Eqs. (A24 A26) allows to conclude e.g. for the canonical correlator

$$
\left.\beta\langle X ; Y\rangle\right|_{T, \mu}=-\left.\int d E \frac{\partial f}{\partial E} \beta\langle X ; Y\rangle\right|_{T=0, \mu=E_{\mathrm{F}}=E} .
$$

A similar equation holds for the dynamic correlator. Therefore, for additive (non-interacting) Fermion systems we can always restrict the discussion to the zero temperature limit.

In the zero frequency limit further simplifications are possible. For example, any autocorrelator is essentially determined by states at the Fermi level $E_{\mathrm{F}}$,

$$
\beta(X \| X)(T=0)=\pi \hbar \operatorname{Tr}\left\{X \delta\left(E_{\mathrm{F}}-H\right) X \delta\left(E_{\mathrm{F}}-H\right)\right\}
$$

which shows that the longitudinal conductivity is a Fermi level quantity.

To simplify other correlators it is convenient to use the equation of motion on the level of the one-particle resolvent operator

$$
G(z) \dot{X} G\left(z^{\prime}\right)=\frac{i}{\hbar}\left\{G(z) X-X G\left(z^{\prime}\right)+\left(z-z^{\prime}\right) G(z) X G\left(z^{\prime}\right)\right\} .
$$

An important example for an (essentially) Fermi level quantity is the zero frequency dynamic correlator of two operators one of which is a time derivative

$$
\beta(\dot{X} \| Y)(T=0)=\beta\langle\Delta X ; \Delta Y\rangle(T=0)+\lim _{\epsilon \rightarrow+0} \frac{\epsilon}{\pi} \operatorname{Tr}\left\{X G^{-}\left(E_{\mathrm{F}}\right) Y G^{+}\left(E_{\mathrm{F}}\right)\right\} .
$$

The d.c. conductance coefficients at $T=0$ read for $n \neq m$

$$
G_{k m}(\omega=0, T=0)=\frac{-\hbar}{2 \pi} \operatorname{Tr}\left\{G^{-}\left(E_{\mathrm{F}}\right) \dot{Q}_{m} G^{+}\left(E_{\mathrm{F}}\right) \dot{Q}_{n}\right\}
$$

and it is obvious from Eq. (A30) that the conductance coefficients are Fermi level quantities, too.

By applying Eq. (A30) to the longitudinal conductivity one finds

$$
\sigma_{x x}(\omega=0, T=0)=\frac{4 e^{2}}{h L^{d}} \epsilon^{2} \operatorname{Tr}\left\{G^{-} G^{+} x^{2}-G^{-} x G^{+} x\right\}\left(E_{\mathrm{F}}\right) .
$$

From Eq. A31) one can conclude that the conductance coefficients are determined by asymptotic Green's functions, and it is tempting to formulate a scattering theoretical description. This is indeed possible by decomposing the total Hamiltonian into two parts, one 
of which describes the leads, $H_{\text {leads }}^{0}$, and a second part which describes the conductor plus its coupling to the leads, $H_{\text {scatter, }}$

$$
H=H_{\text {leads }}^{0}+H_{\text {scatter }}
$$

The total resolvent $G(E)$ can then be decomposed according to Dyson's formula on introducing the $T$-matrix of scattering theory

$$
G(E)=G^{0}(E)+G^{0}(E) T(E) G^{0}(E), \quad T(E)=H_{\text {scatter }} G(E)\left(G^{0}(E)\right)^{-1} .
$$

The eigenstates of the leads are scattering states, denoted as $|k, \alpha\rangle$, where $k$ labels the lead and $\alpha$ the quantum number (channel) of a scattering state at energy $E$. The matrix elements

$$
t_{k m}^{\alpha \beta}(E):=\langle m, \beta|T(E)| k, \alpha\rangle
$$

define transmission amplitudes to scatter from channel $\alpha$ in lead $k$ to channel $\beta$ in lead $m$ and form the matrix of transmission amplitudes $t_{k n}$. Applying Eq. (A30) and Eq. (A31) and using the fact that the asymptotic charge operators are nothing but $-e$ times the projector onto the Hilbert space of the leads, the important Büttiker formula $(m \neq k)$ [20]

$$
G_{k m}(\omega=0, T=0)=\frac{e^{2}}{h} \operatorname{Tr}\left\{t_{k m} t_{k m}^{\dagger}\right\}\left(E_{\mathrm{F}}\right)
$$

can be obtained. 


\section{REFERENCES}

[1] E. Abrahams, P. W. Anderson, D. C. Liciardello, T. V. Ramakrishnan, Phys. Rev. Lett. 42, 673 (1979).

[2] A. Abrikosov, Fundamentals of the Theory of Metalls, North-Holland, Amsterdam (1988).

[3] A. Altland, D. Fuchs, Phys. Rev. Lett. 74, 4269 (1995).

[4] B.L. Al'tshuler, B.I. Shklovskii, Sov. Phys. JETP 64, 127 (1986).

[5] B.L. Al'tshuler, V. E. Kravtsov, I. V. Lerner, Sov. Phys. JETP 64, 1352 (1986); Phys. Lett. A 134, 488 (1989).

[6] B. L. Al'tshuler, I. K. Zharekeshev, S. A. Kotochigova, B. I. Shklovskii, Sov. Phys. JETP 67, 625 (1988).

[7] B. L. Al'tshuler, P. A. Lee, R. A. Webb (Eds.), Mesoscopic Phenomena in Solids, North-Holland, New York (1991).

[8] P. W. Anderson, Phys. Rev. 109, 1492 (1958).

[9] P. W. Anderson, D. J. Thouless, E. Abrahams, D. S. Fisher, Phys. Rev. B 22, 3519 (1980).

[10] T. Ando, Phys. Rev. B 40, 5325 (1989).

[11] H. Aoki, J. Phys. C 16, L205 (1983); Phys. Rev. B 33, 7310 (1986).

[12] H. Aoki, T. Ando, Phys. Rev. Lett. 54, 831 (1985).

[13] N. Argaman, Y. Imry, U. Smilansky, Phys. Rev. B 47, 4440 (1993).

[14] A.G. Aronov, V.E. Kravtsov, I.V. Lerner, Phys. Rev. Lett. 74, 1174 (1995).

[15] C. W. J. Beenakker, H. van Houten, Quantum Transport in semiconductor nanostructures, in: H. Ehrenreich, D. Turnbull (Eds.), Solid States Physiscs, 44, Academic Press, New York (1991).

[16] C.W.J. Beenakker, Phys. Rev. Lett. 70, 1155 (1993).

[17] C.W.J. Beenakker, B. Rejaei, Phys. Rev. Lett. 71, 3698 (1993).

[18] G. Bergmann, Phys. Rep. 107, 1 (1984).

[19] W. Beyer, Ph.D. thesis, Universität Marburg (1974).

[20] M. Büttiker, Phys. Rev. Lett. 57, 1761 (1986). 
[21] M. Büttiker, T. Christen, cond-mat/9601075.

[22] J.L. Cardy, J. Phys.A 17, L385 (1984).

[23] M. Caselle, Phys. Rev. Lett. 74, 2776 (1995).

[24] C. Castellani, L. Peliti, J. Phys. A 19, L429 (1986).

[25] M.E. Cates, J.M. Deutsch, Phys. Rev. A 35, 4907 (1987).

[26] J.T. Chalker, P.D. Coddington, J. Phys.C 21, 2665 (1988).

[27] J.T. Chalker, G.J. Daniell, Phys. Rev. Lett. 61, 593 (1988).

[28] J.T. Chalker, Physica A 167, 253 (1990).

[29] J.T. Chalker, M. Janssen, D.G. Polyakov, unpublished (1995).

[30] J.T. Chalker, private communication (1995).

[31] J.T. Chalker, A. Dohmen, Phys. Rev. Lett. 75, 4496 (1995).

[32] J.T. Chalker, I.V. Lerner, R.S. Smith, Phys. Rev. Lett. 77, 554 (1996).

[33] J.T. Chalker, V.E. Kravtsov, I.V. Lerner, JETP Lett. 64, 386 (1996).

[34] S. Cho, P. A. Fisher, Phys. Rev. B 55, 1637 (1997).

[35] D. H. Cobden, E. Kogan, Phys. Rev. B 54, 17316 (1996).

[36] A. Cohen, Y. Roth, B. Shapiro, Phys. Rev. B 38, 12125 (1988); A. Cohen, B. Shapiro, Int. J. Mod. Phys. 6, 1243 (1992).

[37] A. Crisanti, G. Paladin, A. Vulpiani, Products of Random Matrices, Springer-Verlag, Berlin Heidelberg (1993).

[38] S. Datta, Electronic Transport in Mesoscopic Systems, Cambridge University Press, Cambridge (1995).

[39] A. Dohmen, Diploma thesis, Universtät zu Köln, unpublished (1995).

[40] A. Dohmen, P. Freche, M. Janssen, Phys. Rev. Lett. 76, 4207 (1996).

[41] G. J. Dolan, G. D. Osheroff, Phys. Rev. Lett. 43, 721 (1979).

[42] O.N. Dorokhov, Sov. Phys. JETP 58, 606 (1983); P.A. Mello, P. Pereyra, N. Kumar, Ann. Phys. 181, 290 (1988).

[43] F.J. Dyson, J. Math. Phys. 13, 90 (1972).

[44] I. Edrei, M. Kaveh, B. Shapiro, Phys. Rev. Lett. 62, 2120 (1989). 
[45] J. T. Edwards, D. J. Thouless, J. Phys. C 5, 807 (1972).

[46] K.B. Efetov, K. B. Efetov, Adv. Phys., 32, 53 (1983)

[47] K.B. Efetov, V.N. Prigodin, Phys. Rev. Lett. 70, 1315 (1993).

[48] S.N. Evangelou, T. Ziman, J. Phys. C 20, L235 (1987).

[49] S. N. Evangelou, Phys. Rev. B 49, 16805 (1994).

[50] V.I. Falko, K.B. Efetov, Europhys. Lett. 32, 627 (1995), Phys. Rev. B 52, 17413 (1995).

[51] U. Fastenrath, G. Adams, R. Bundschuh, T. Hermes, B. Raab, I. Schlosser, T. Wehner, T. Wichmannn, Physica A 172302 (1991).

[52] U. Fastenrath, PhD thesis, Universität zu Köln, Shaker-Verlag, Aachen (1992).

[53] U. Fastenrath, M. Janssen, W. Pook, Physica A 191, 401 (1992).

[54] M. Feingold, Y. Avishai, R. Berkovits, Phys. Rev. B 52, 8400 (1995).

[55] H.A. Fertig, Phys. Rev. B 38, 996 (1988).

[56] E. Fick, G. Sauermann, The Quantum Statistics of Dynamic Processes, SpringerVerlag, Berlin (1990).

[57] H. Fürstenberg, Trans. Am. Math. Soc. 108, 337 (1963).

[58] Y.V. Fyodorov, A.D. Mirlin, Phys. Rev. Lett. 67, 2405 (1991); Phys. Rev. Lett. 69, 1093 (1992); Int. J. Mod. Phys. B 83795 (1994); Phys. Rev. B 51, 13403 (1995); A.D. Mirlin Y.V. Fyodorov, Phys. Rev. Lett. 72526 (1994); J. Phys. France I, 4, 665 (1994).

[59] L. P. Gor'kov, A. I. Larkin, D. E. Khmel'nitskii, JETP Lett. 30, 228 (1979).

[60] T.C. Halsey, M.H. Jensen, L.P. Kadanoff, I. Procaccia, B.I. Shraiman, Phys. Rev. A 33, 1141 (1986).

[61] H. Hegger, B. Huckestein, K. Hecker, M. Janssen, A. Freimuth, G. Reckziegel, R. Tuzinski, Phys. Rev. Lett. 77, 3885 (1996).

[62] M. Henneke, B. Kramer, T. Ohtsuki, Europhys. Lett. 27, 389 (1994).

[63] S. Hikami, A. I. Larkin, Y. Nagaoka, Prog. Theor. Phys. 63, 707 (1980).

[64] E. Hofstetter, M. Schreiber, Phys. Rev. B 48, 16979 (1993).

[65] E. Hofstetter, M. Schreiber, Phys. Rev. B 49, 14726 (1994). 
[66] B. Huckestein, L. Schweitzer, B. Kramer, Surface Sciences 263, 125 (1992);

[67] B. Huckestein, L. Schweitzer, Phys. Rev. Lett. 72, 713 (1994).

[68] B. Huckestein, Rev. Mod. Phys. 67, 357 (1995).

[69] Y. Imry, in: G. Grinstein, G. Mazenko (Eds.), Directions in Condensed Matter Physiscs, World Scientific, Singapore (1986).

[70] M. Janssen, Solid State Commun. 79, 1073 (1991).

[71] M. Janssen, Int. J. Mod. Phys. B 8, 943 (1994).

[72] M. Janssen, O. Viehweger, U. Fastenrath and J. Hajdu, Introduction to the Theory of the Integer Quantum Hall Effect, VCH, Weinheim (1994).

[73] T. Kawarabayashi, T. Ohtsuki, K. Slevin, Y. Ono, Phys. Rev. Lett. 77, 3593 (1996).

[74] R. Klesse, M. Metzler, Europhys. Lett. 32, 229 (1995).

[75] R. Klesse, PhD thesis, Universität zu Köln, unpublished (1996).

[76] K. von Klitzing, G. Dorda, M. Pepper, Phys. Rev. Lett. 45, 494 (1980).

[77] S. Kobayashi, F. Komori, Y. Ootuka, W. Sasaki, J. Phys. Soc. Jap. 49, 1635 (1980).

[78] E. Kogan, M. Kaveh, Phys. Rev. B 52, 3813 (1995).

[79] B. Kramer, A. MacKinnon, Rep. Prog. Phys. 56, 1469 (1993).

[80] S. V. Kravchenko, W. E. Mason, G. E. Bowker, J. E. Furneaux, V. M. Pudalov, M. D'Iorio, Phys. Rev. B 51,7038 (1995).

[81] V.E. Kravtsov, I.V. Lerner, Phys. Rev. Lett. 74, 2563 (1995).

[82] V.E. Kravtsov, A.D. Mirlin, JETP Lett. 60, 656 (1994).

[83] R. Kubo, M. Toda, N. Hashitsume, Statistical Physics II, Springer-Verlag, Berlin (1985).

[84] R. Landauer, IBM J. Res. Dev. 1, 223 (1957).

[85] R. Landauer, Phil. Mag. 21, 863 (1970).

[86] S.A. van Langen, P.W. Brouwer, C.W.J. Beenakker, Phys. Rev. E 53, R1344 (1996).

[87] D.C. Langreth, A.E. Abrahams, Phys. Rev. B 24, 2978 (1981).

[88] L. A. Lee, T. V. Ramakrishnan, Rev. Mod. Phys. 57, 287 (1985).

[89] A.M.S. Macedo, J.T. Chalker, Phys. Rev. B 46, 14985 (1992). 
[90] A. MacKinnon, B. Kramer, Phys. Rev. Lett. 47, 1546 (1981).

[91] P. Markos,B. Kramer, Phil. Mag. B 68, 357 (1993).

[92] M.L. Mehta, Random Matrices, Academic, New York 2nd ed. (1991).

[93] P.A. Mello, E. Akkermans, B. Shapiro, Phys. Rev. Lett. 61, 459 (1988).

[94] P. A. Mello, S. Tomsovic, Phys. Rev. Lett. 67, 342 (1991).

[95] V.I. Melnikov, Fiz. Tverd, Tela 23, 782; Soviet Phys. Solid St. 23, 444 (1981).

[96] M. Metzler, PhD thesis, Universität zu Köln, unpublished (1996).

[97] A.D. Mirlin, JETP Lett. 62, 603 (1995); Phys. Rev. B 53, 1186 (1996).

[98] A.D. Mirlin, cond-mat/9512095.

[99] A.D. Mirlin, Y.V. Fyodorov, Phys. Rev. Lett. 72, 526 (1994).

[100] N. F. Mott, E. A. Davis, Electronic Processes in Non-Crystalline Materials (2 edn.), Clarendon Press, Oxford (1979).

[101] B.A. Muzykantskii, D.E. Khmelnitskii, Phys. Rev. B 51, 5480 (1995).

[102] T.M. Nieuwenhuizen, M.C.W. van Rossum Phys. Rev. Lett. 74,2674 (1995).

[103] V.I. Oseledec, Trans. Moscow Math. Soc. 19, 197 (1968).

[104] M. Paalanen, R. N. Bhatt, Physica B 169, 223 (1991).

[105] G. Paladin, A. Vulpiani, Physics Reports 156, 147 (1987).

[106] R. Peierls, Z. Phys. 80, 763 (1933).

[107] J.-L. Pichard, G. Sarma, J. Phys.C 14, L127 (1981).

[108] J-L. Pichard in: K.A. Benedict, J.T. Chalker (Eds.), Localisation 1990, IOP Publishing, Bristol (1991).

[109] W. Pook, M. Janssen, Z. Phys. B 82, 295 (1991)

[110] K. Pracz, M. Janssen, P. Freche, J. Phys.: Condensed Matter 8, 7147 (1996).

[111] R. Prange, S. Girvin (Eds.), The Quantum Hall Effect, Springer, Berlin (1990).

[112] H. Risken, The Fokker-Planck Equation, Springer-Verlag, Berlin (1989).

[113] M.P. Sarachik in: P.P. Edwards, C.N.R. Rao (Eds.), Metal-Insulator Transitions Revisited, Taylor \& Francis, London (1995).

[114] B. Shapiro, Phys. Rev. Lett. 48, 823 (1982). 
[115] B. Shapiro, Ann. Israel phys. Soc. 5, 367 (1983).

[116] B. Shapiro, Phil. Mag. B 56, 1031 (1987).

[117] M. Schreiber, H. Grußbach, Phys. Rev. Lett. 67, 607 (1991).

[118] L. Schweitzer, J. Phys.: Condensed Matter 7, L281 (1995).

[119] B. I. Shklovskii, B. Shapiro, B. R. Sears, P. Lambrianides, H. B. Shore, Phys. Rev. B 47, 11487 (1993).

[120] I.E. Smolyarenko, B.L. Al'tshuler, cond-mat/9606181.

[121] A.D. Stone, P.A. Mello, K.A. Muttalib, J.-L. Pichard in: B.L. Al'tshuler, P.A. Lee, R.A. Webb, Mesoscopic Phenomena in Solids, North-Holland, Amsterdam (1991).

[122] H. Stupp M. Hornung, M. Lakner, O. Madel, H. von Löhneysen, Phys. Rev. Lett. 71, 2634 (1993).

[123] D. J. Thouless, Phys. Rep. 13 C, 93 (1974)

[124] G. Timp , Surf. Sci. 196, 68 (1988).

[125] D.C. Tsui: 4.DFG-Rundgespräch über den Quanten Hall Effekt, Schleching, Germany (1989).

[126] V.N. Tutubalin, Theor. Prob. Appl. 13, 65 (1968).

[127] A.D. Virster, Theor. Prob. Appl. 15, 667 (1970).

[128] Z. Wang, B. Jovanovic, D.-H. Lee, Phys. Rev. Lett. 77, 4426 (1996).

[129] S. Washburn, R. A. Webb, Adv. Phys. 35, 375 (1986).

[130] S. Washburn, R. A. Webb, Rep. Prog. Phys. 55, 1311 (1992).

[131] F. Wegner, Z. Phys. B 25, 327 (1976).

[132] F. Wegner, Z. Phys. B 35, 207 (1979).

[133] F. Wegner, Z. Phys. B 36, 209 (1980)

[134] E.P. Wigner, Ann. Math. 53, 36 (1951).

[135] I. K. Zharekeshev, B. Kramer, Jpn. J. Appl. Phys. 34, 4361 (1995). 


\section{Figure Captions}

Figure 1: Schematic view of a mesoscopic conductor. Electrons experience elastic scattering events on their way from the current source to the current sink.

Figure 2: A mesoscopic ring-shaped conductor. A flux $\phi$ is put through the ring.

Figure 3: Resistance oscillations for a ring-shaped conductor with flux $\phi$. The period is $\phi_{0}=h / e$ as shown in the inset where the Fourier spectrum is displayed. (after [124]).

Figure 4: Universal conductance fluctuations as a function of applied magnetic field. Different curves correspond to gold-nanowires of different length. The average value of conductance was shifted for convinience (after [61]).

Figure 5: Hopping transport in amorphous Silicon. The logarithm of the conductivity is plotted versus $T^{-1 / 4}$ showing Mott's law. The different curves are for different deposition temperatures (after [19]).

Figure 6: The resistance of thin disordered film of coupled fine $\mathrm{Cu}$-particles as function of logarithm of the temperature (after [77]).

Figure 7: Diffusion path in a disordered system. The electron propagates in both directions (time reversal symmetry). Due to constructive interference the quantum return probability is twice as great as the classical return probability.

Figure 8: The resistance curves of a thin Mg-film (upper set of curves) as function of magnetic field (magneto-resistance). The weak localization occurs for zero field. After a superposition with 1/100 atomic layer of $\mathrm{Au}$ the magneto-resistance changes drastically. The $\mathrm{Au}$ introduces a rather pronounced amount of spin-orbit scattering which rotates the spin in the complementary scatterd waves. This changes the interference from constructive to destructive (after [18]).

Figure 9: Conductivity as a function of electron concentration indicating strong localization (after 122]).

Figure 10: The quantum Hall effect: The Hall resistance $\rho_{x y}$ and the magnetoresistance $\rho_{x x}$ as function of the magnetic field measured in medium mobility GaAs heterostructures at $60 \mathrm{mK}$ (after [125]).

Figure 11: Schematic view of a multi-probe conductor. Currents $I_{k}$ entering the system from probe $k$ are counted positive. 
Figure 12: Schematic view of a box shaped conductor in d dimensions characterized by length $L$ and cross section $L_{t}^{d-1}$.

Figure 13: Schematic view of energy branches for semi-infinite leads. Due to transversal quantization a discrete number of modes exist (characterized by a wave number $k$ ) for a given value of the Fermi energy $E_{F}$.

Figure 14: Scattering in a quasi-1D system.

Figure 15: Adding two 1D conductors in series.

Figure 16: The scattering element of a 2D network. For unit amplitude on the ingoing link from the left and all other ingoing links being empty the amplitudes on the outgoing links are $r_{L}, r_{R}$ and $r$, respectively.

Figure 17: The network resulting from the elements of Fig. 16.

Figure 18: The basic scattering elements of the Chalker-Coddington model.

Figure 19: The Chalker-Coddington network model for 2D disordered electrons in strong magnetic fields. For strong reflection to the left (right) the electrons perform chiral loops.

Figure 20: Qualitative behavior of the $\beta$-function for disordered electrons. For $d=2$ the full line corresponds to absence of magnetic fields and spin-orbit scattering while the dotted line corresponds to the presence of spin-orbit scattering. In $d=3$ a LD transition occurs quite generally at some critical value $g^{*}$ which forms an unstable fixed point of the flow determined by the $\beta$-function.

Figure 21: The $\beta$-function as following from Eq. (169). The curves correspond to (from below) $d=1,2,2.2,3$.

Figure 22: Qualitative behavior of multifractal exponents. The top figure shows the exponents $\tau(q)$ corresponding to moments of a multifractal distribution, the middle shows the resulting generalized dimensions $D_{q}=\tau(q) /(q-1)$ and the bottom shows the $f(\alpha)$-spectrum being the Legendre transform of $\tau(q) . f(\alpha)$ describes the scaling of the whole distribution.

Figure 23: Qualitative behavior of the conductance distribution in a metal. Within a range of width $d \approx \sqrt{\langle g\rangle}$ around the average value $\langle g\rangle$ the distribution is Gaussian with universal variance of order 1 . Outside this region the distribution develops log-normal tails.

Figure 24: The scaling function for a 3D quantum Hall system showing a LD transition. $\Lambda(E, M)$ denotes the scaling variable as a function of energy $E$ and system size $M . \xi(E)$ 
denotes the correlation length obtained from a fitting procedure. Different data symbols correspond to different energies and system sizes (after [39]).

Figure 25: Qualitative picture visualizing the explanation of the quantum Hall effect being due to a sequence of localization-delocalization transitions occurring at the Landau energies. $\rho, \sigma_{x x}, \sigma_{y x}$ denote the density of states, the dissipative conductivity and the Hall conductivity, respectively. In finite systems the range of extended states (grey) on the energy scale has finite width. This width is believed to shrink to zero in the thermodynamic limit.

Figure 26: Squared amplitudes of a critical wave function for a system of linear size 200 magnetic lengths. The corresponding $f(\alpha)$ spectrum $(\bullet)$ together with the parabolic approximation $(\cdots)$ and a semi-elliptic approximation $(-)$ are also shown (after [110]).

Figure 27: Histogram of the logarithm of squared amplitudes shown in Fig. 26. The continuous curve is the distribution function following from the corresponding $f(\alpha)$ spectrum with $\alpha_{0}=2.28 \pm 0.02$ (after [110]).

Figure 28: Squared amplitudes of a critical wave function in the Chalker-Coddington network model.(after [75]).

Figure 29: Histogram of the logarithm of squared amplitudes shown in Fig. 28. The continuous curve is the distribution function following from the parabolic approximation to the $f(\alpha)$ spectrum with $\alpha_{0}=2.27 \pm 0.02$. The inset shows the histogram for the squared amplitudes itself (after 96]).

Figure 30: The function of critical exponents $z(q)$ following from the the scaling relation Eq. (279) (after 110).

Figure 31: Qualtative behavior of the typical local density of states reflecting a localizationdelocalization transition at critical Fermi energy $E^{*}$. 


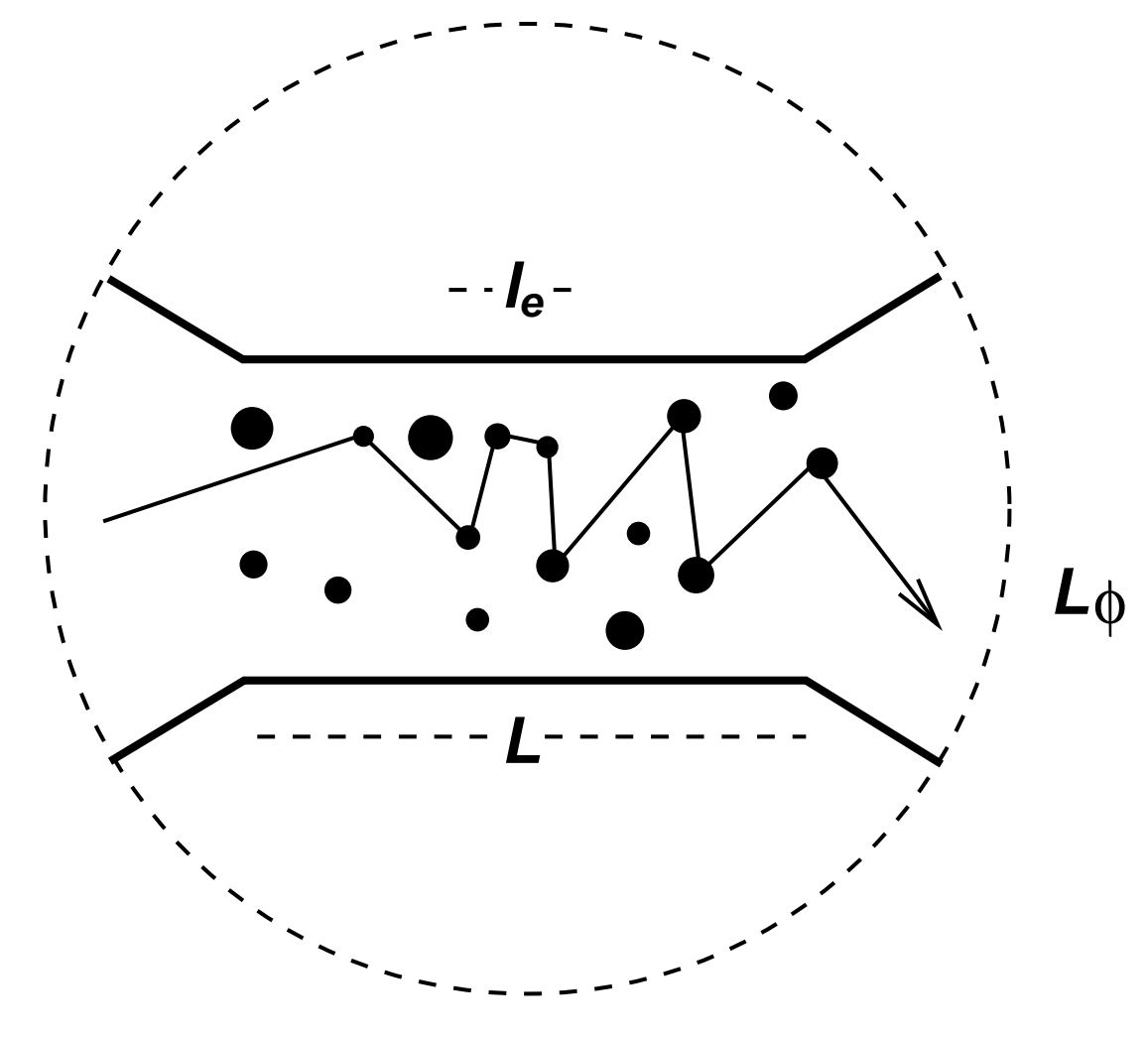




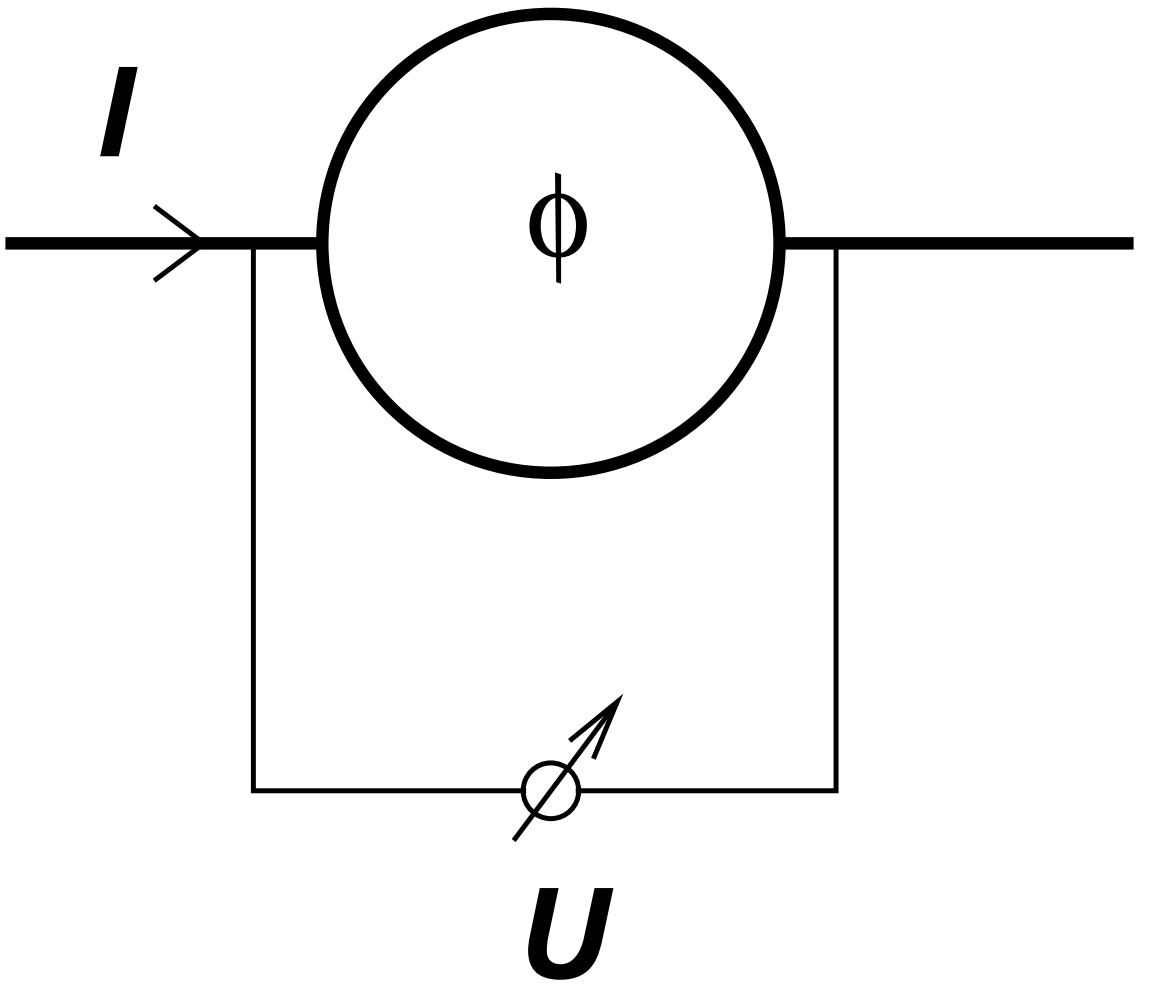




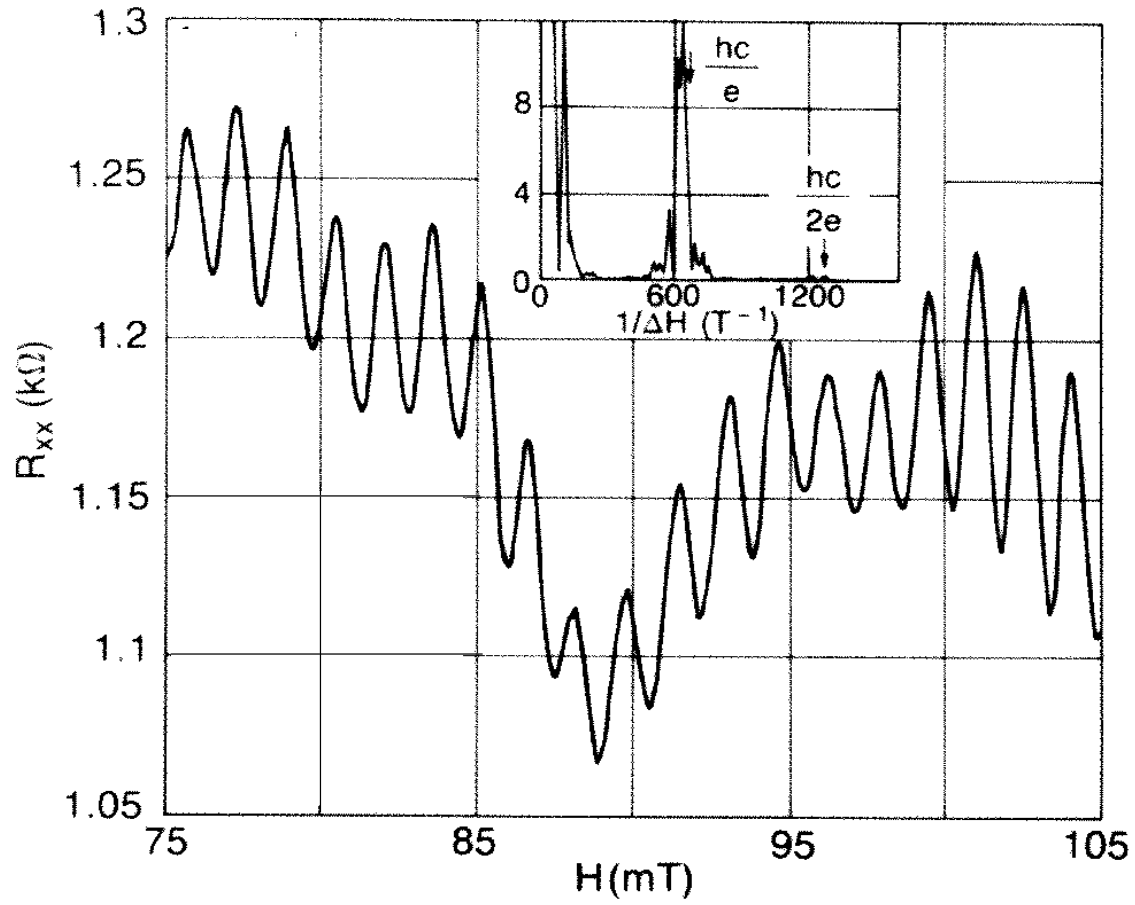




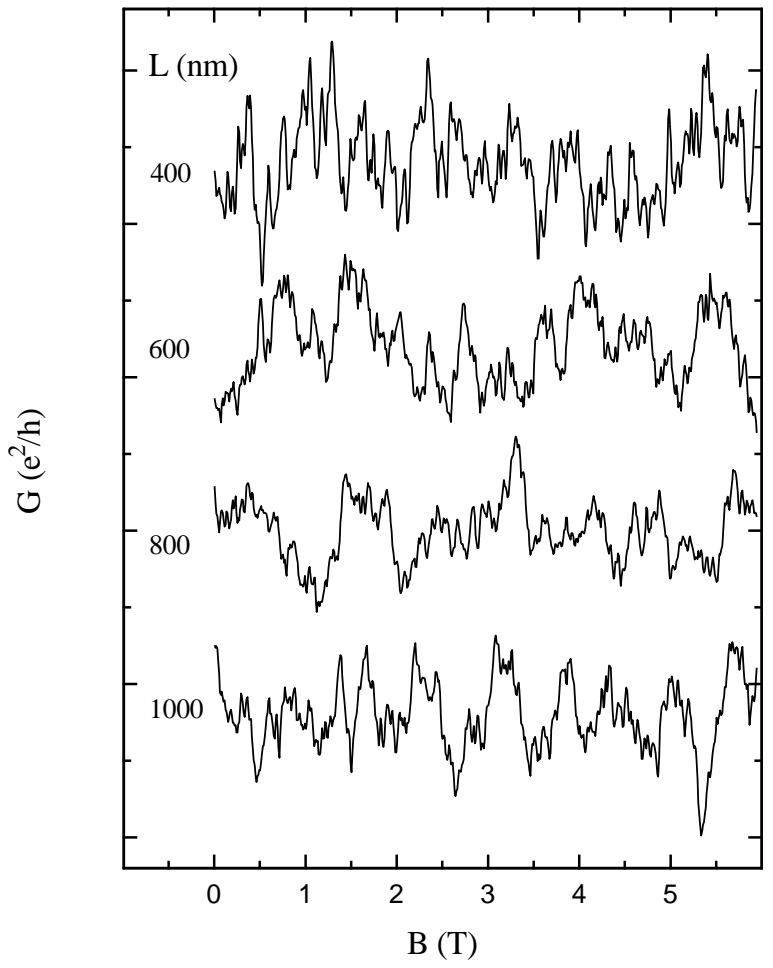




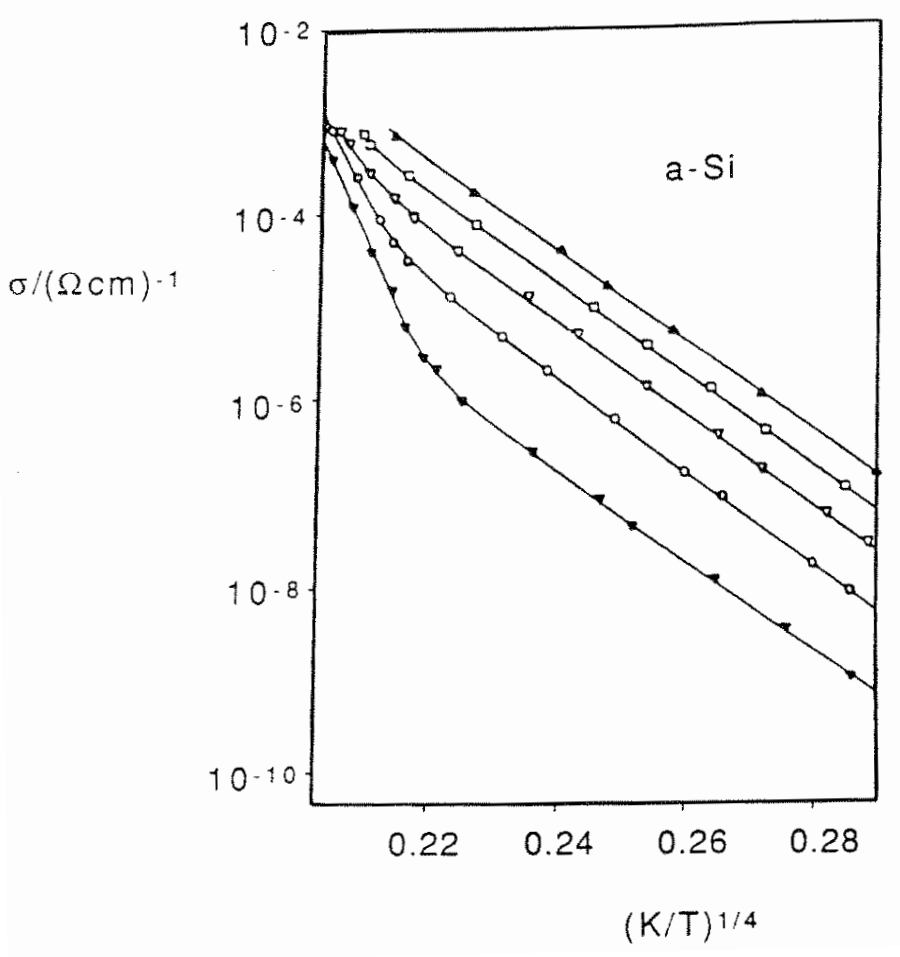




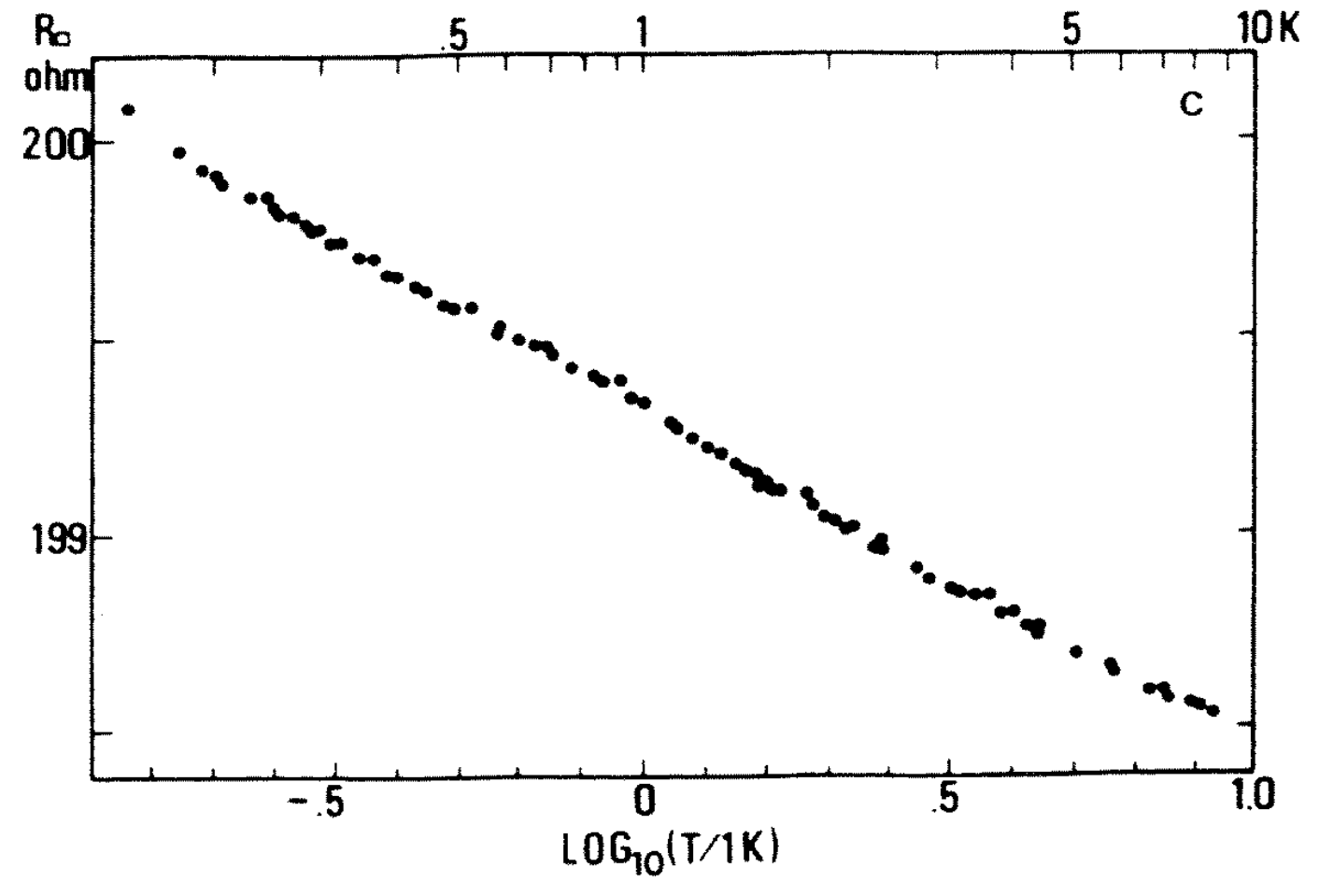




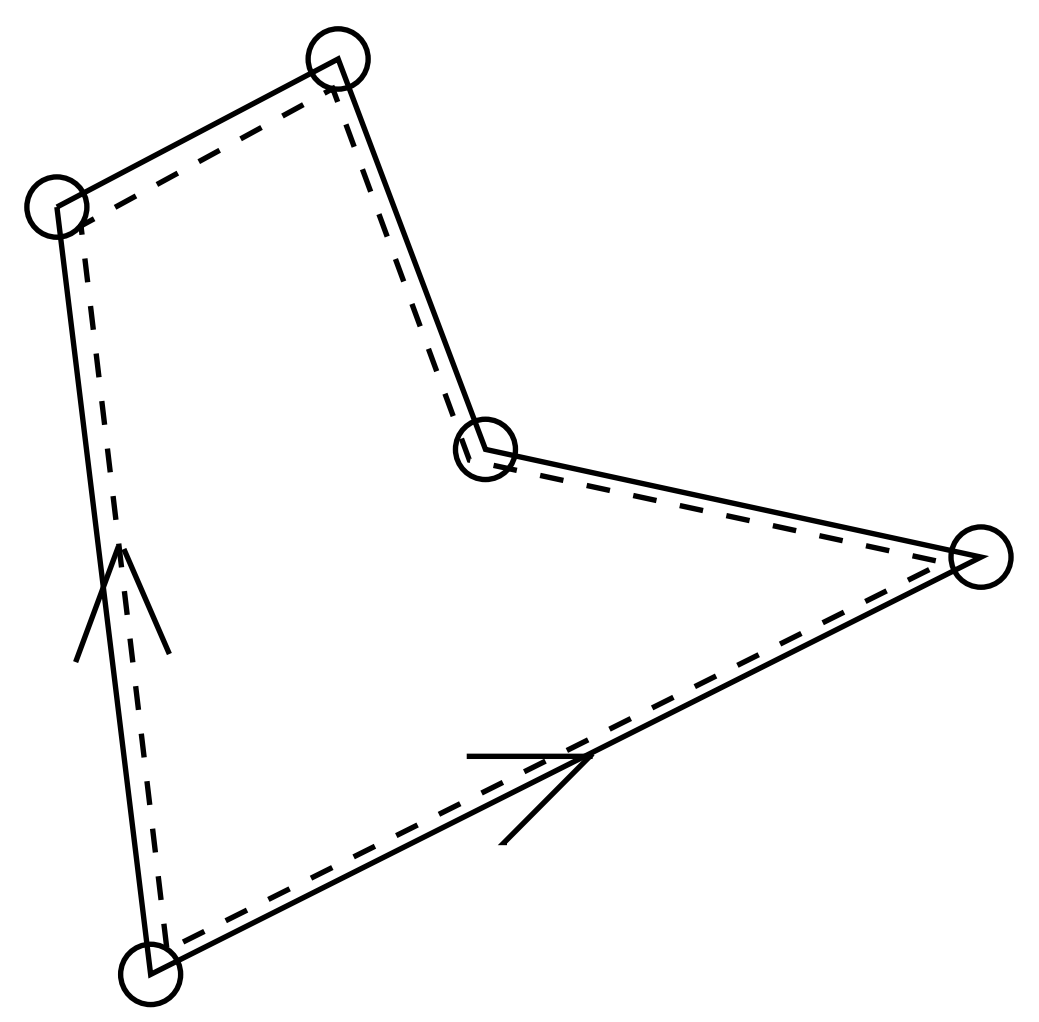




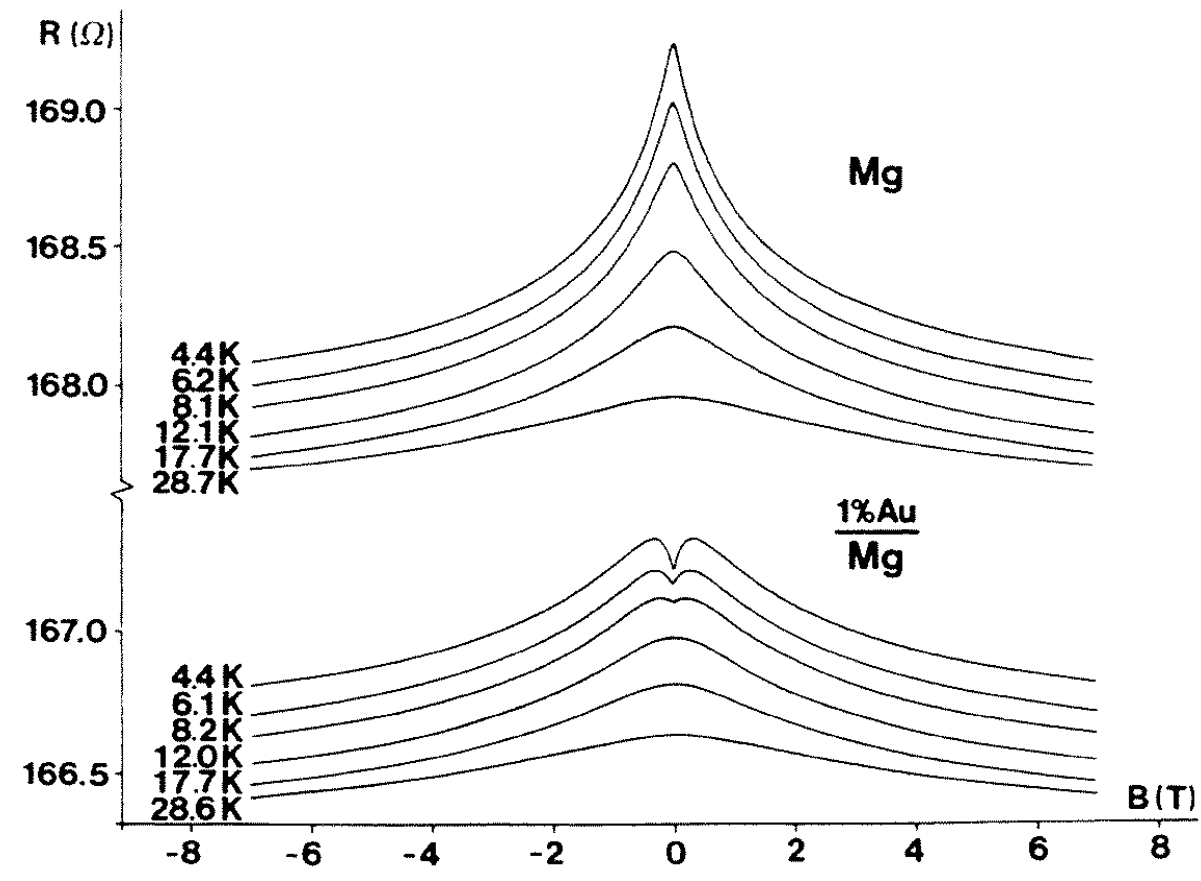




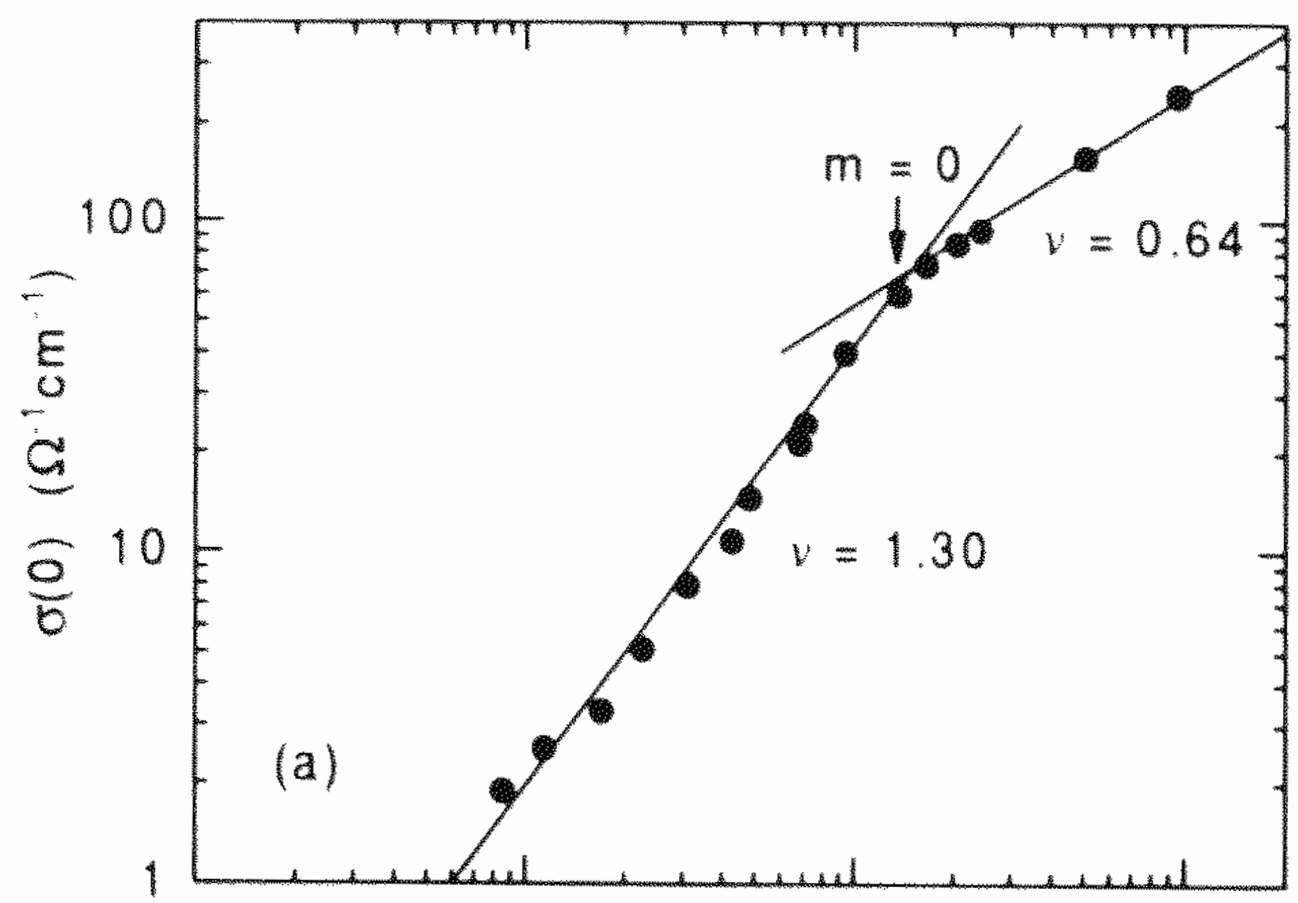




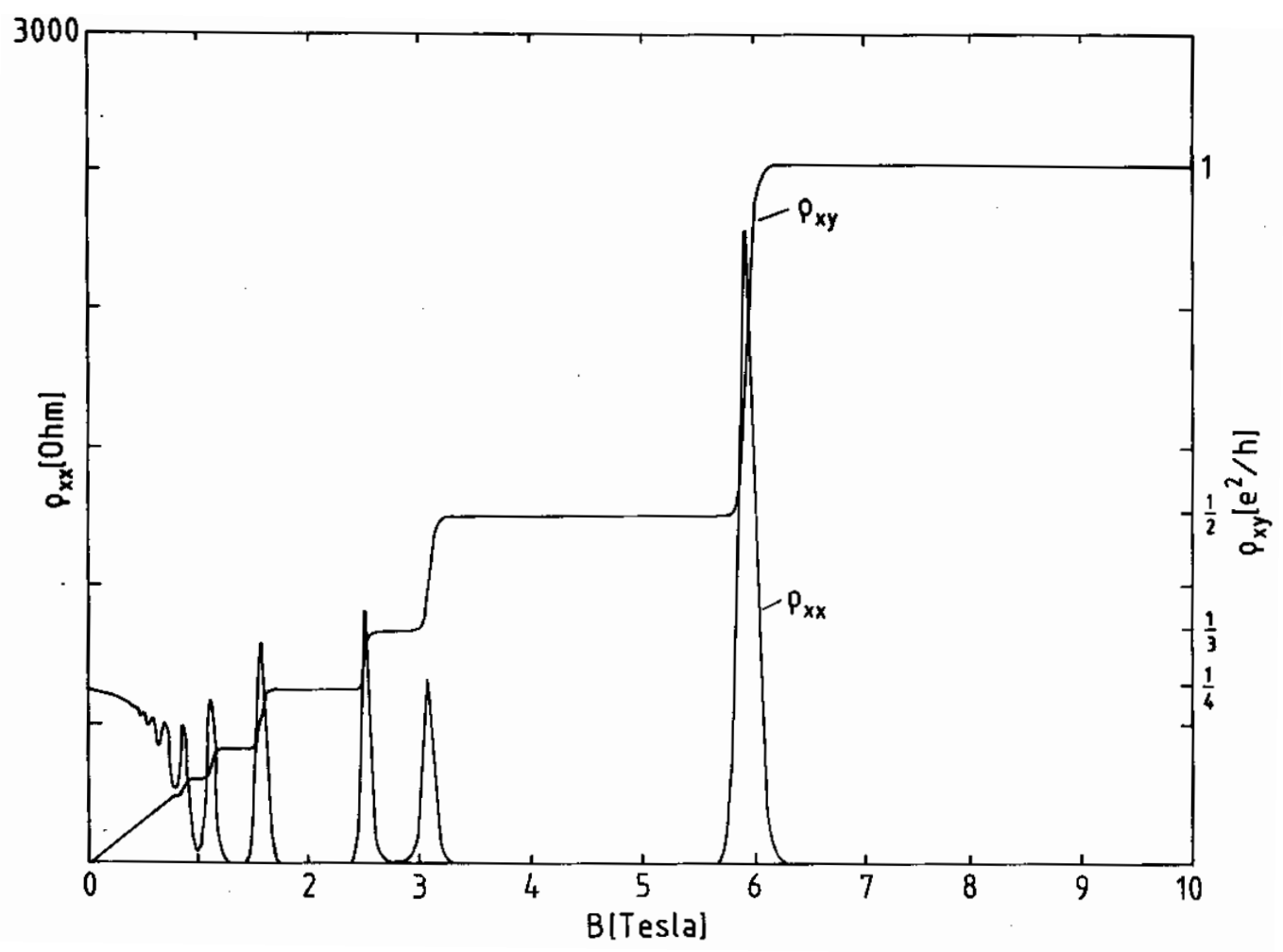




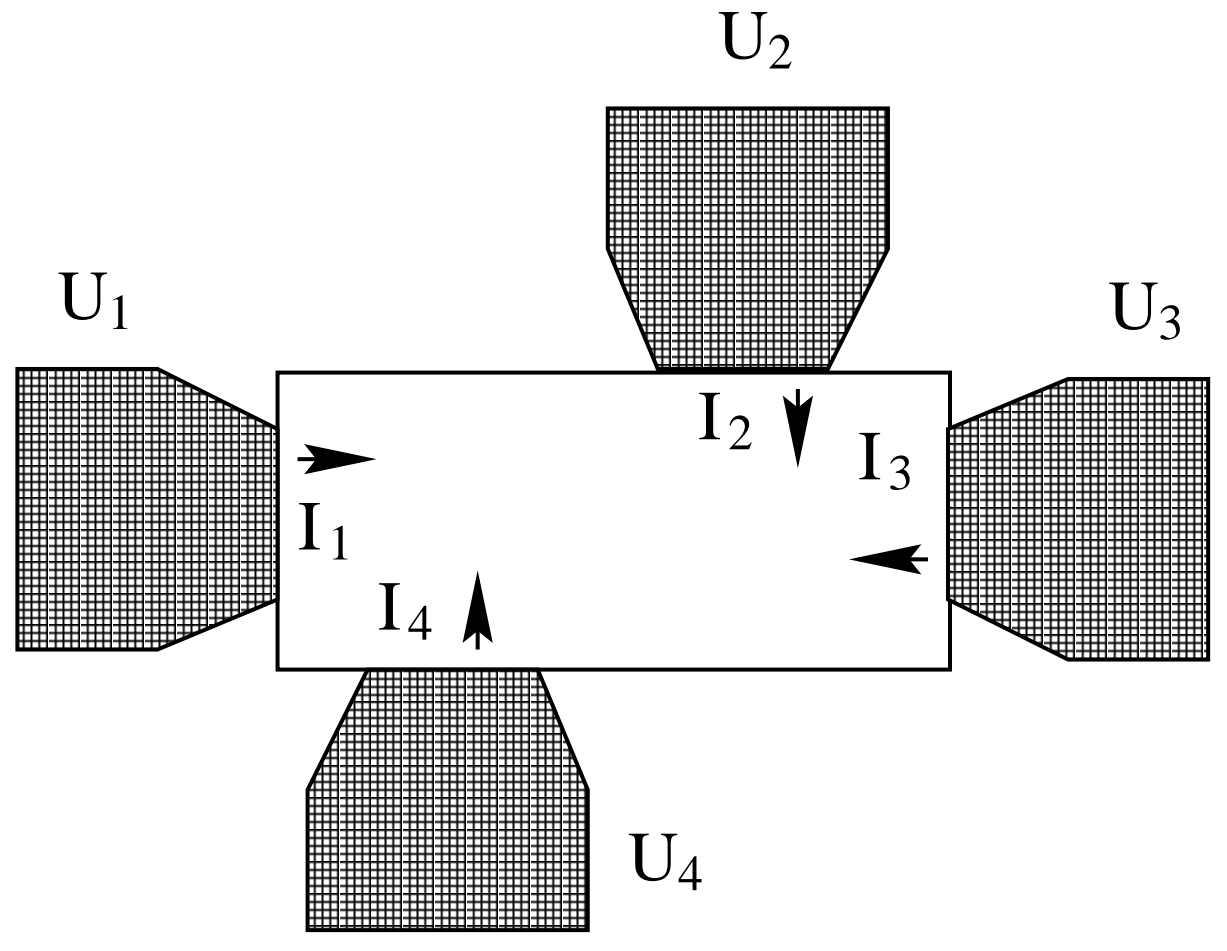




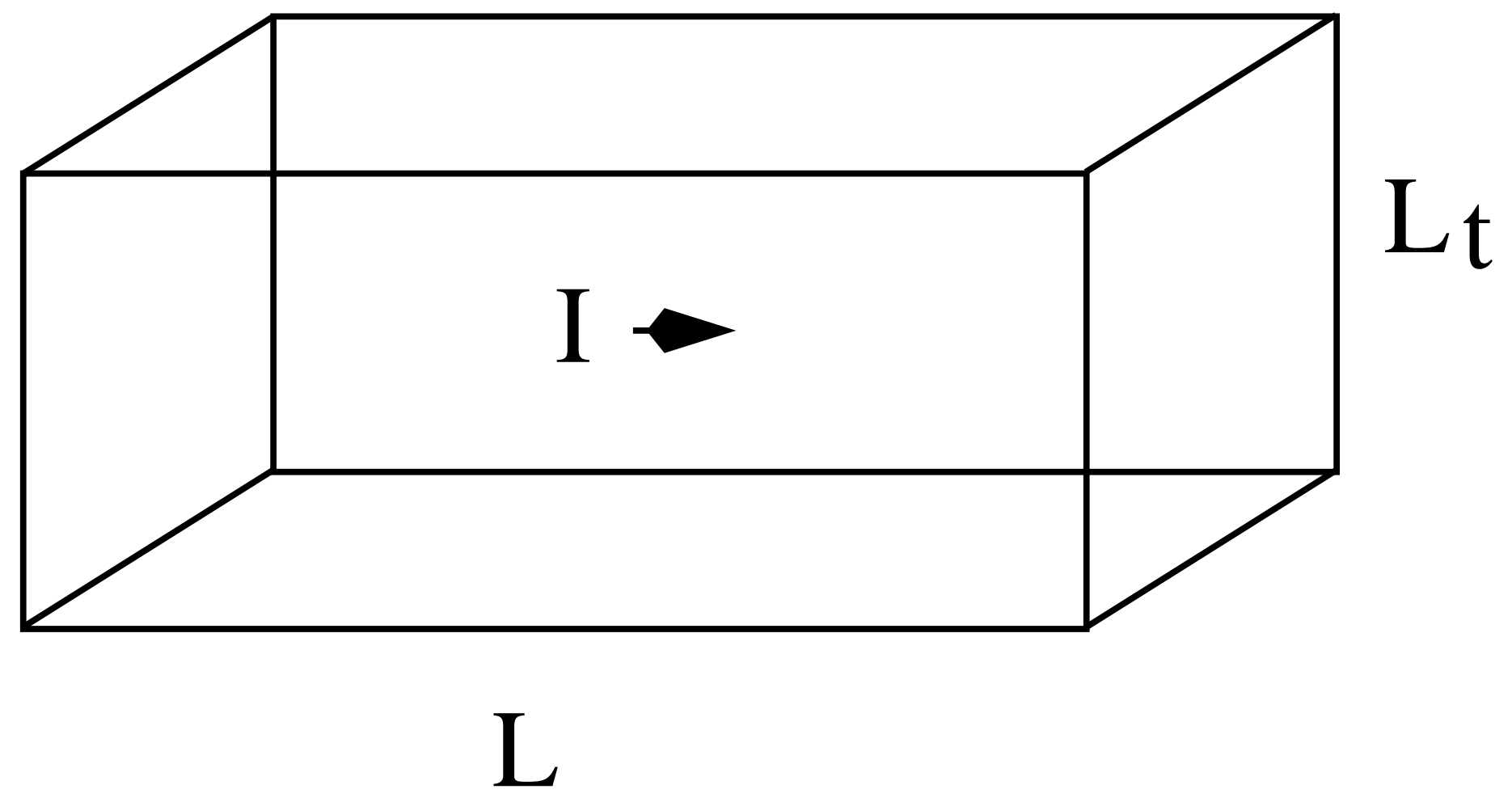




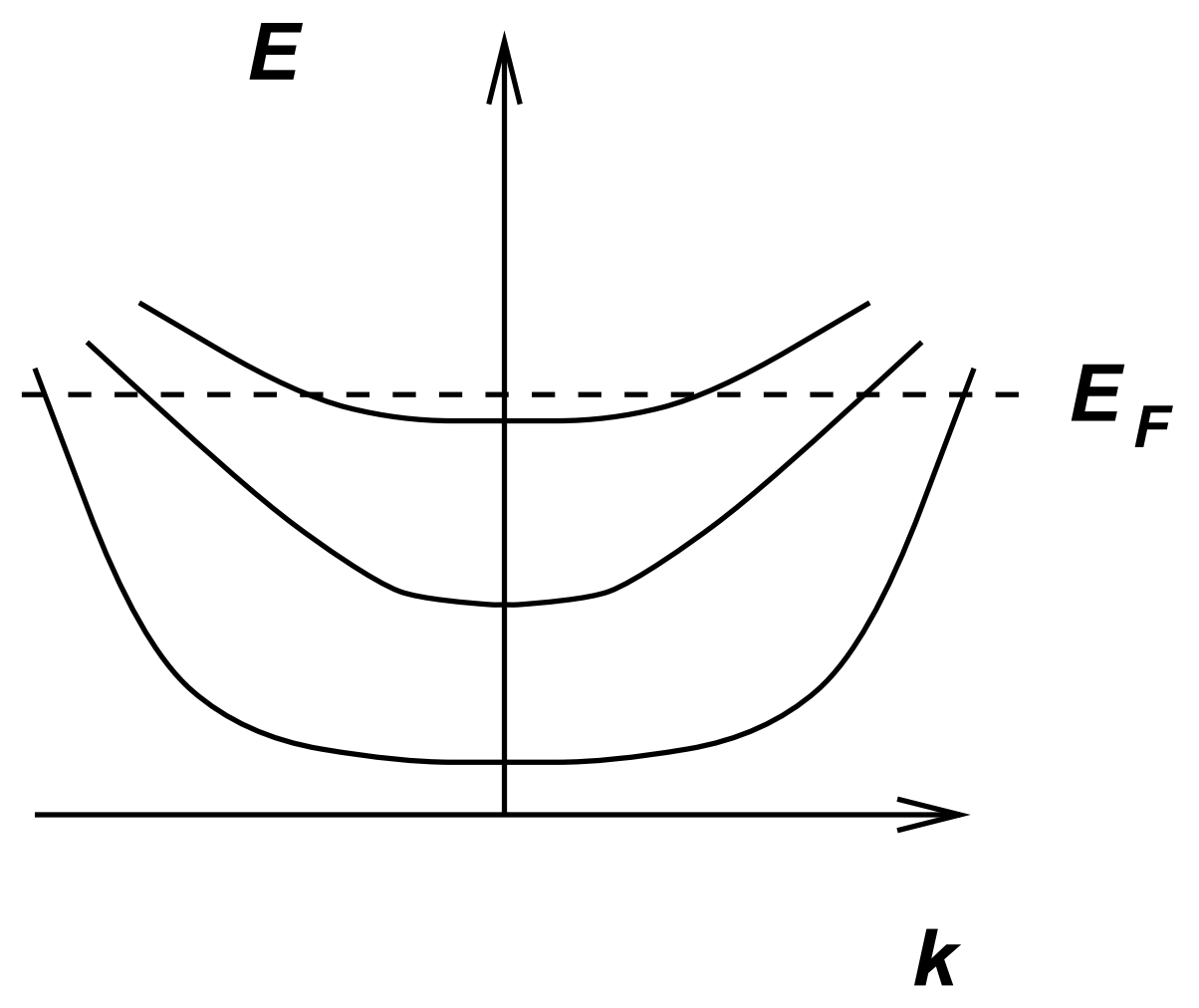




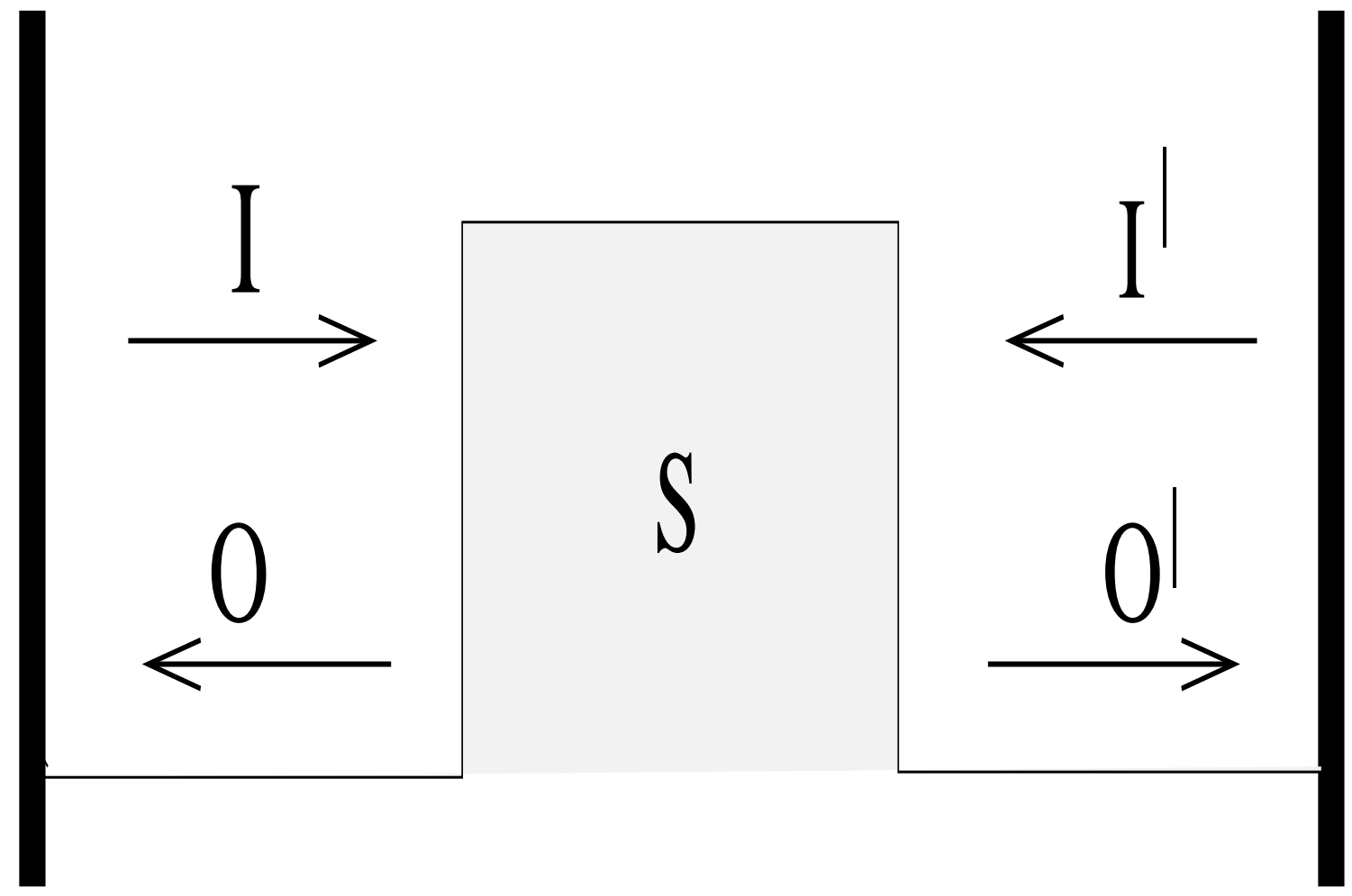



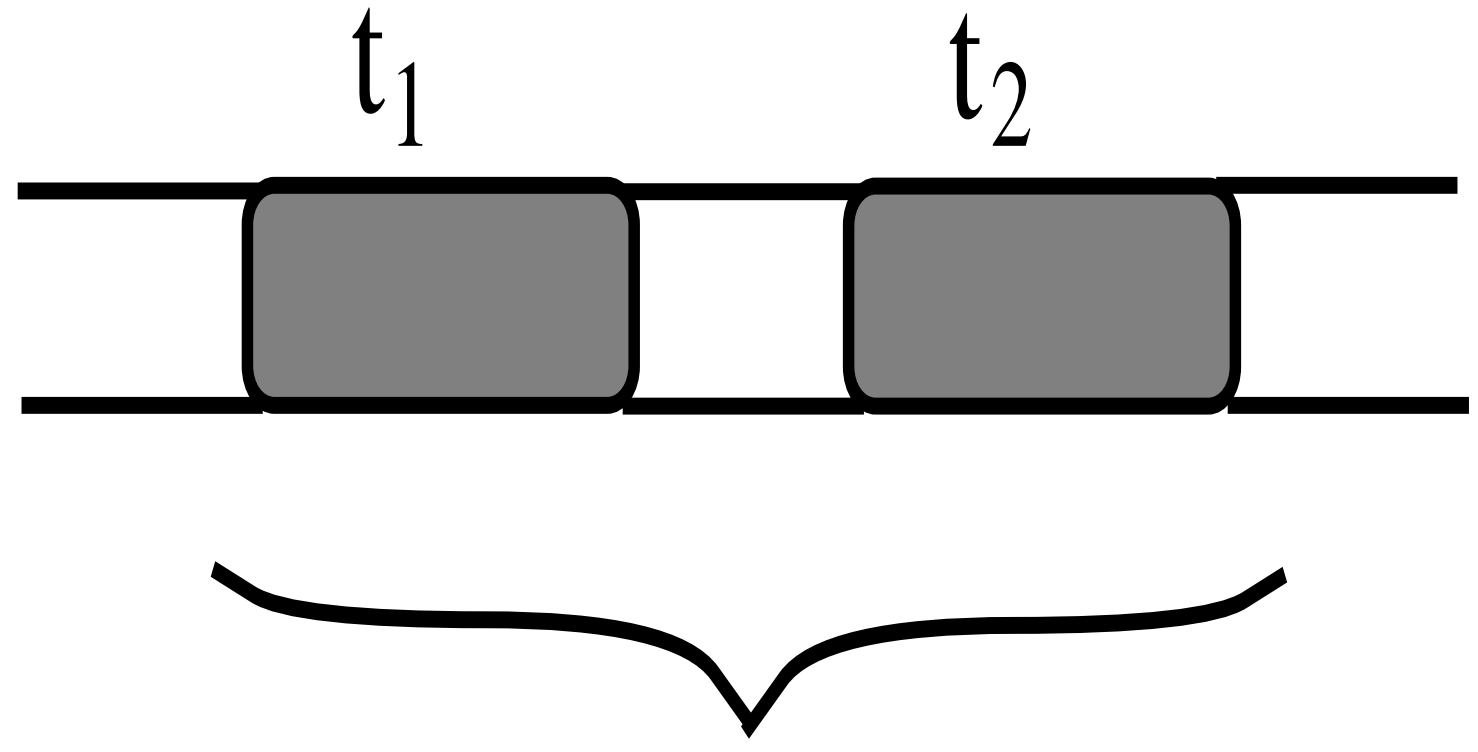

$t_{12}$ 


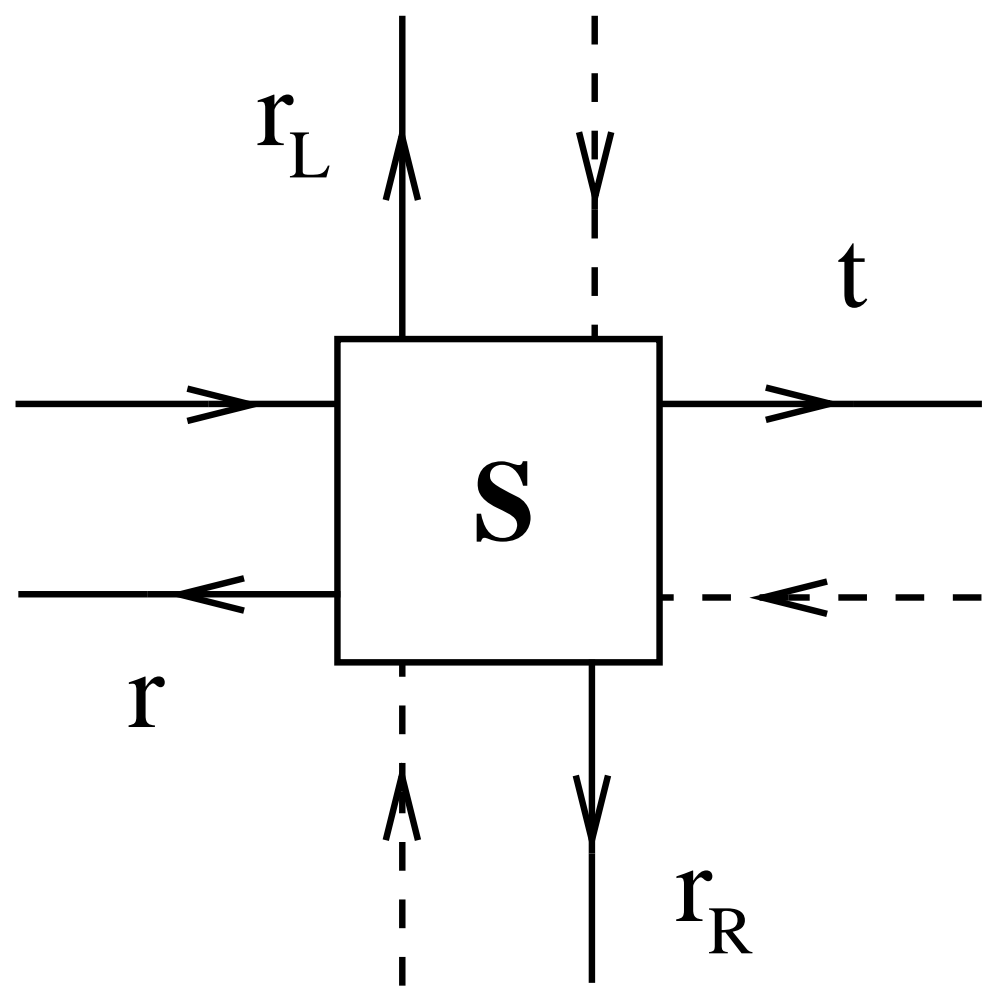




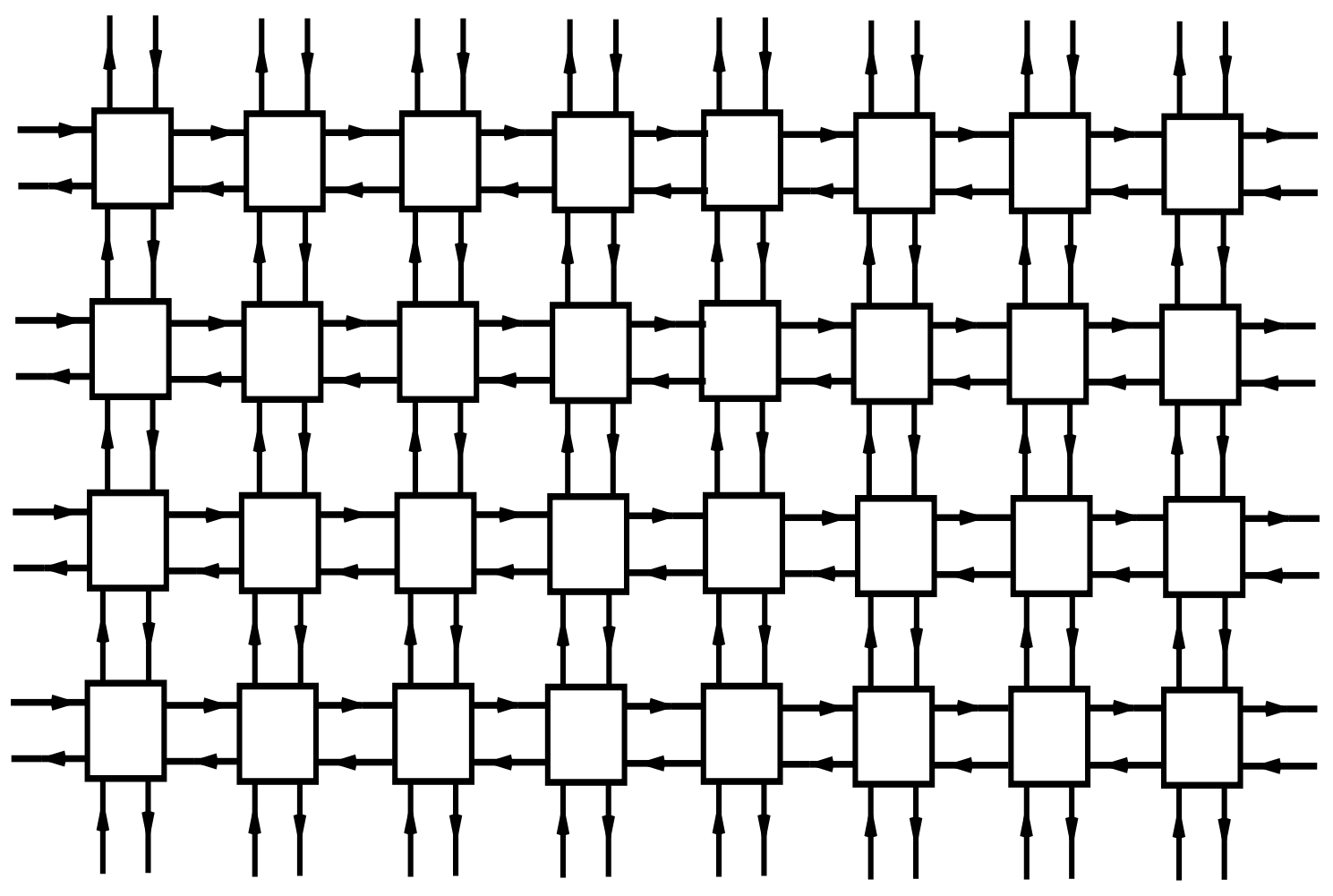




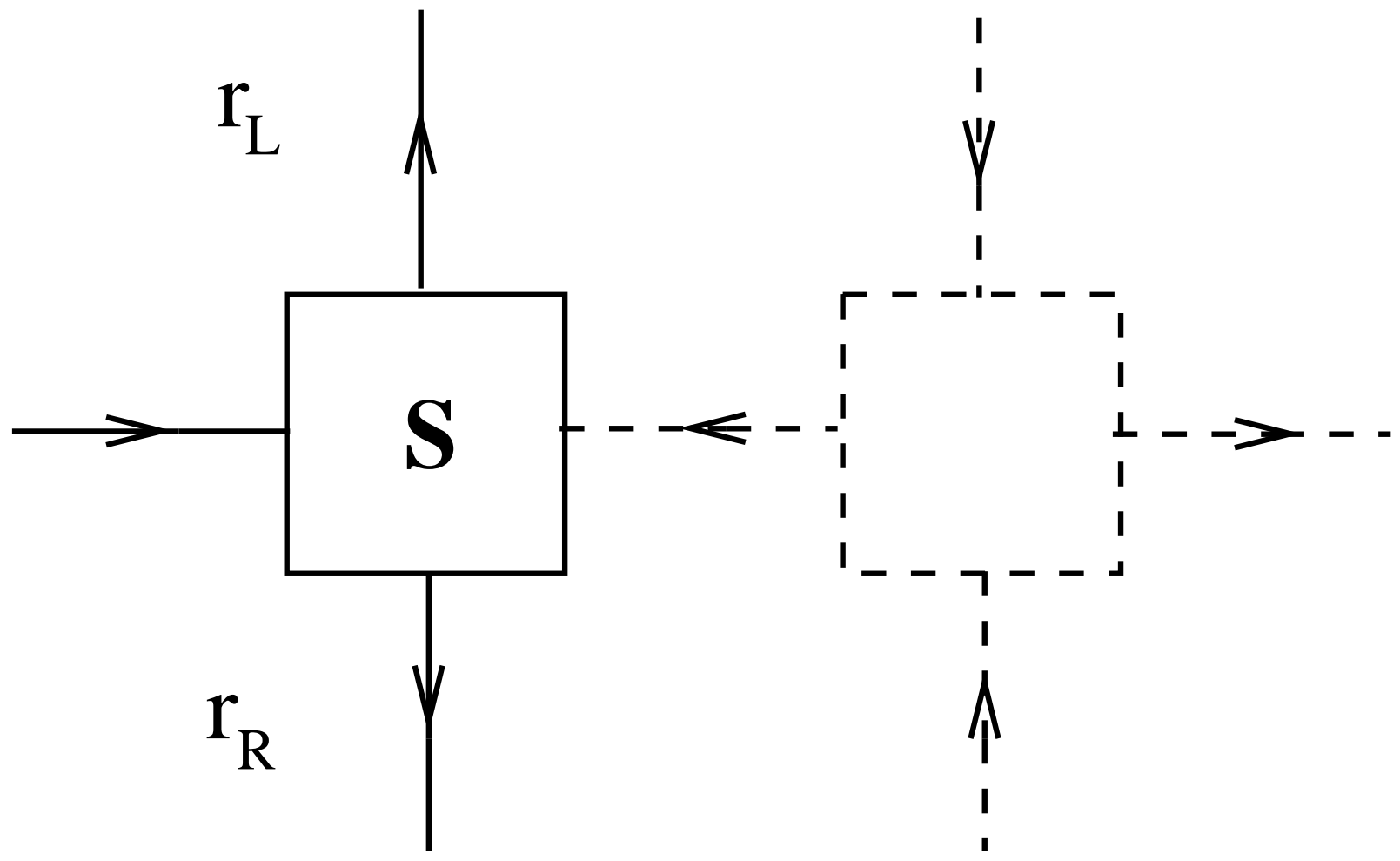




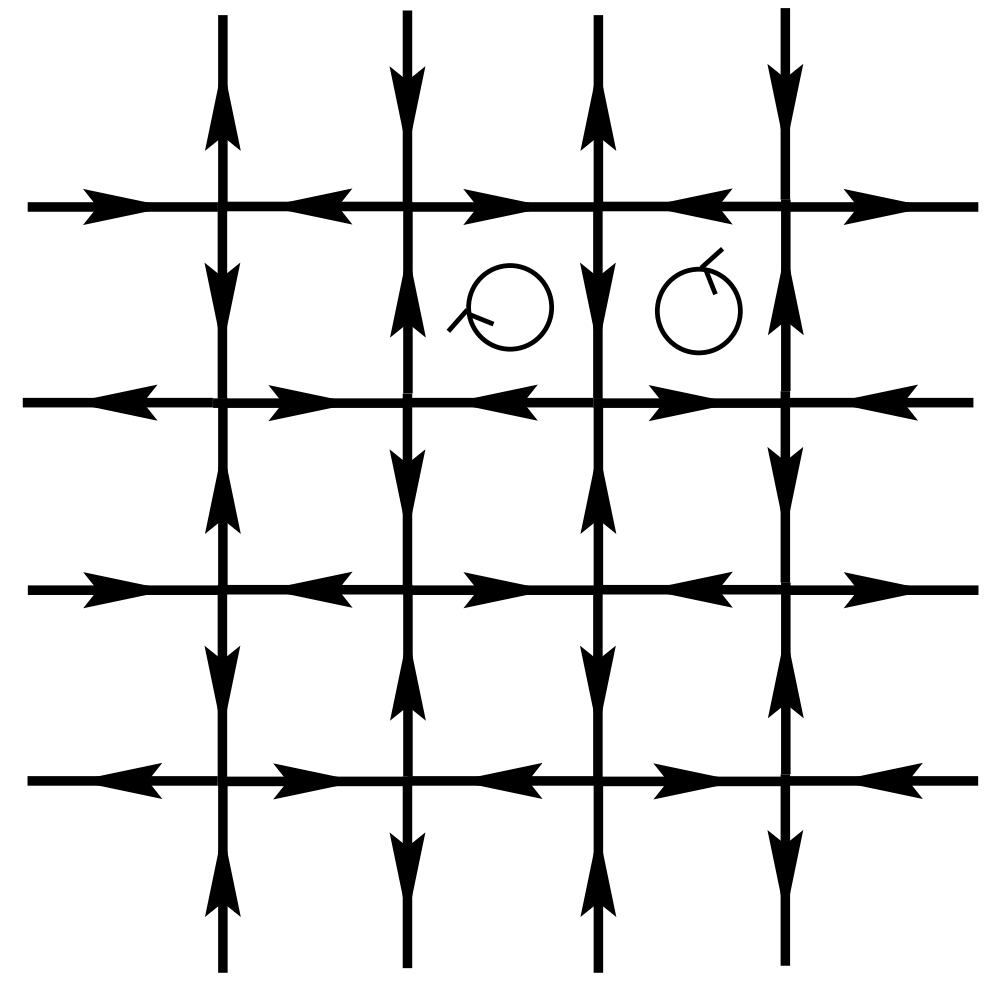



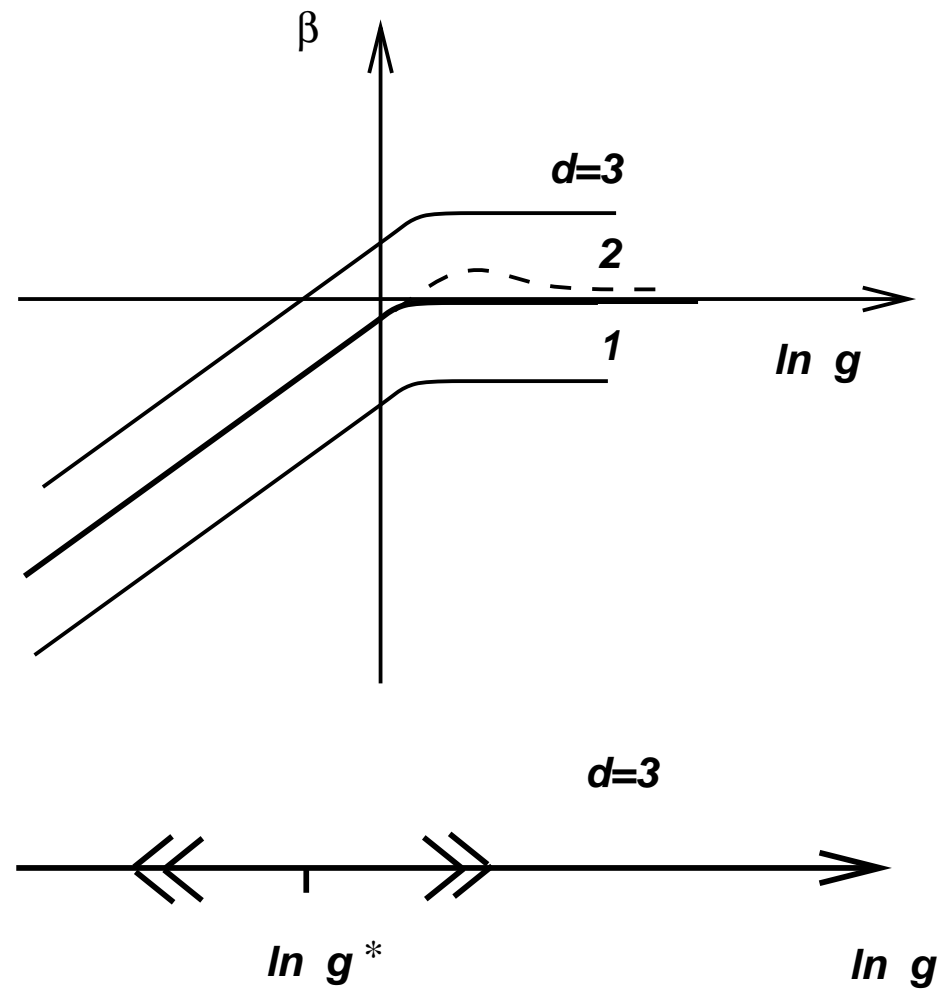


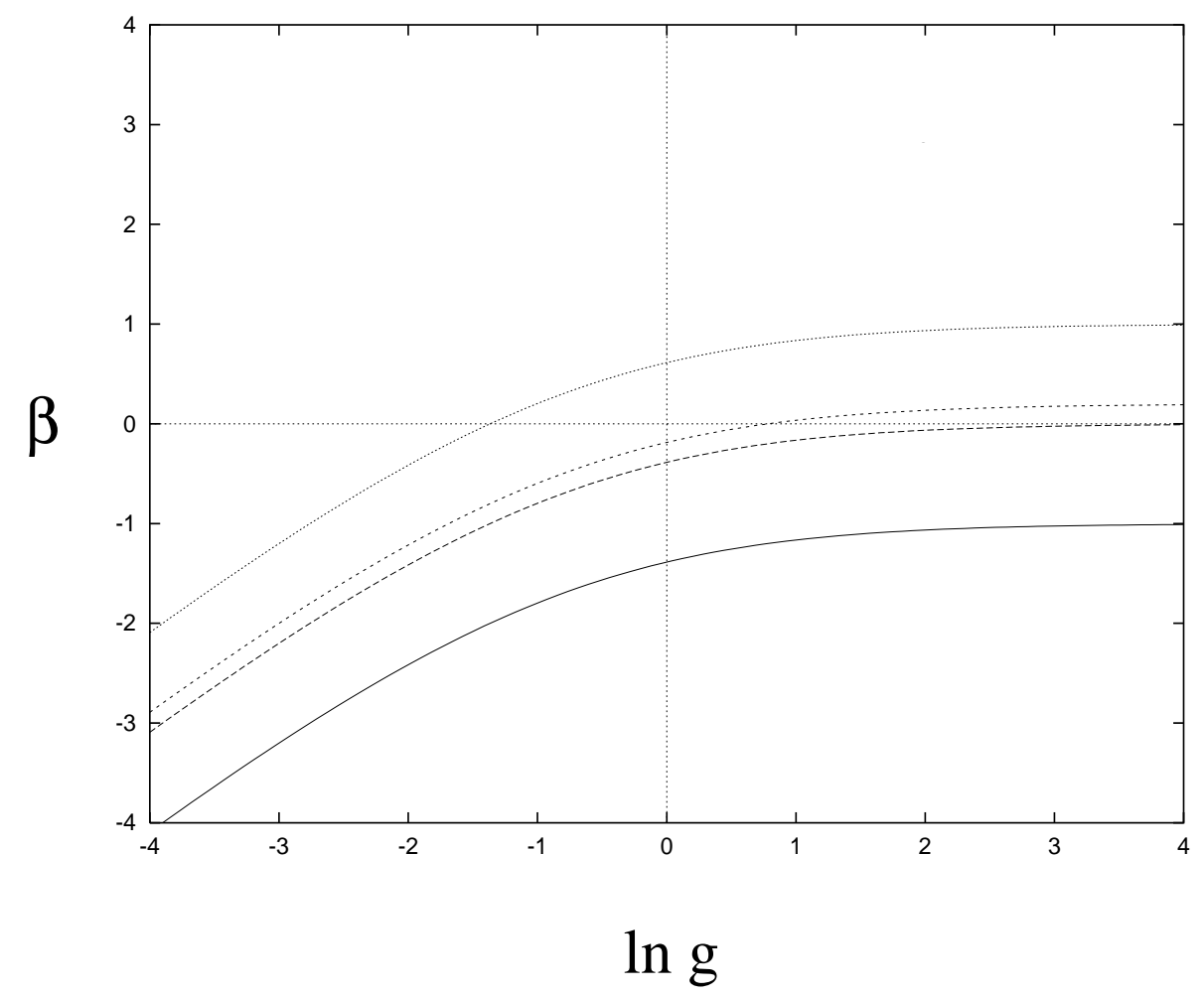



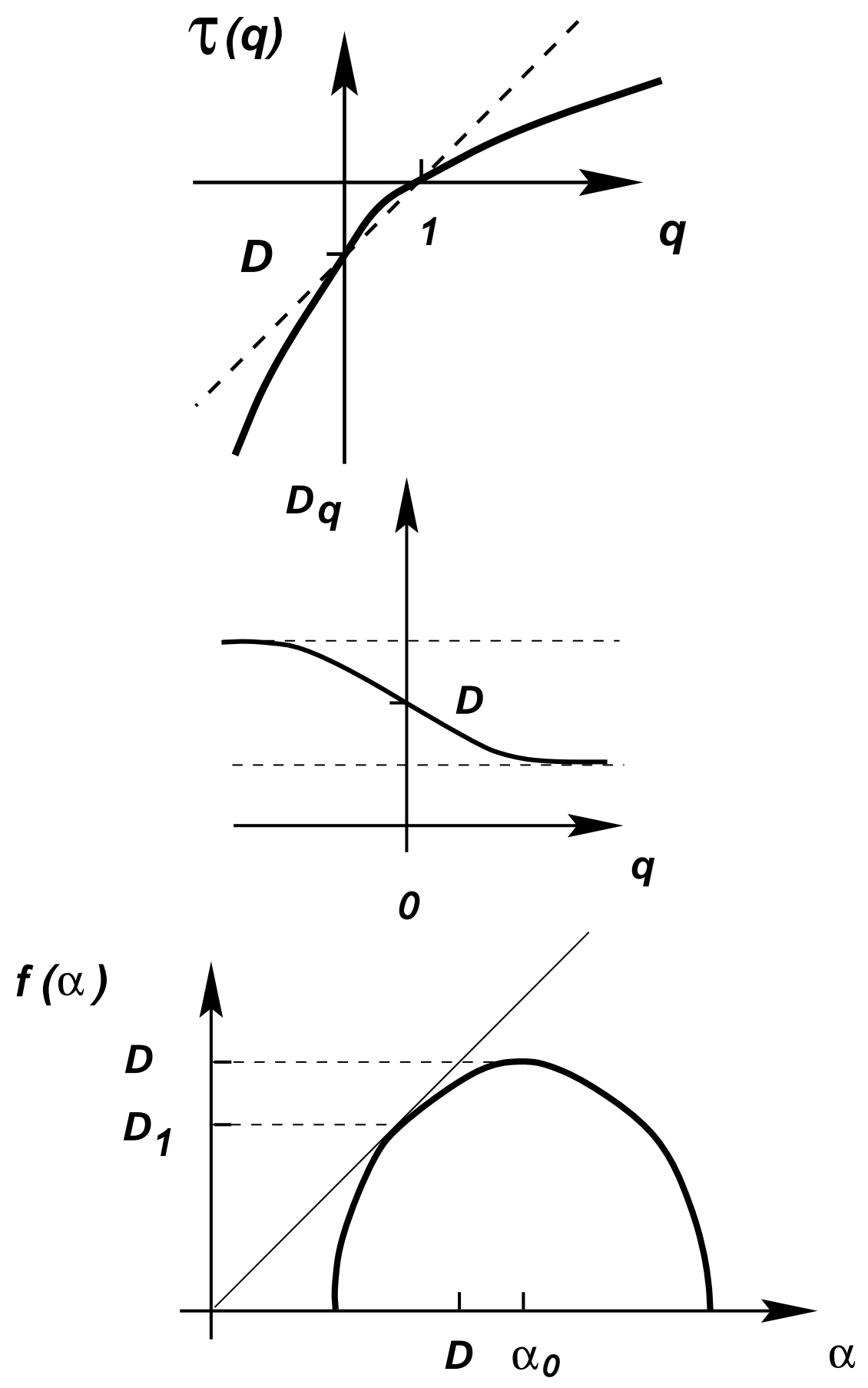


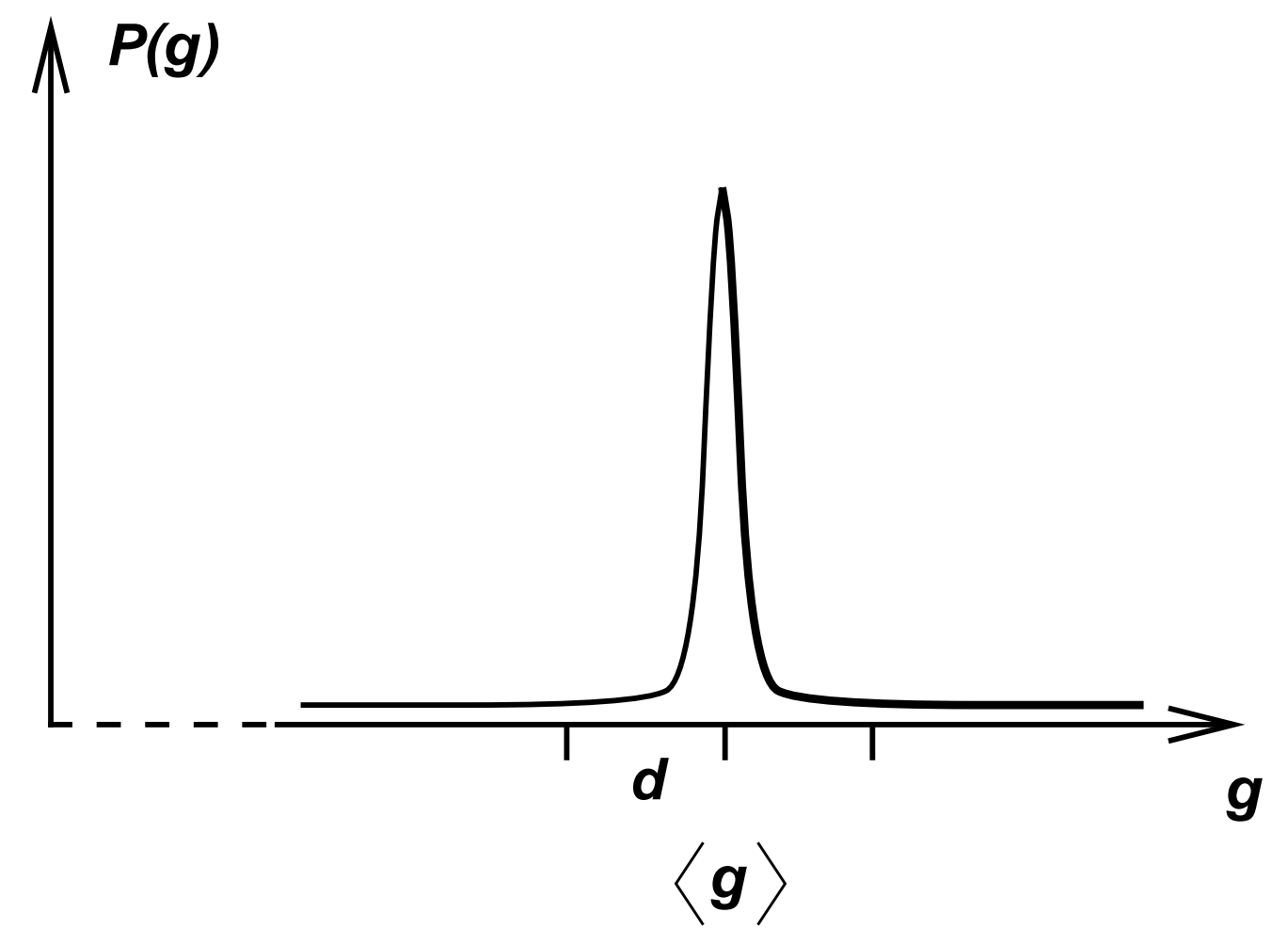




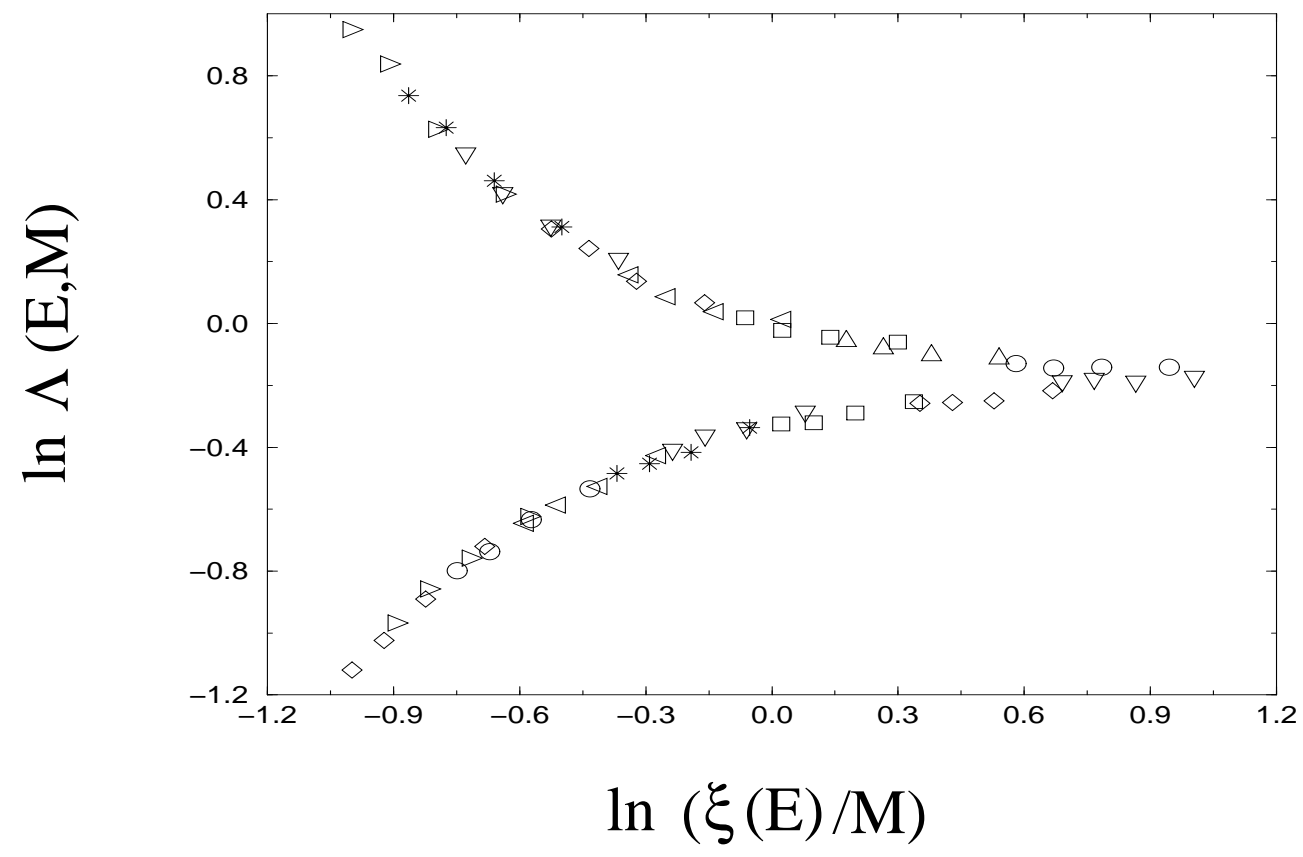




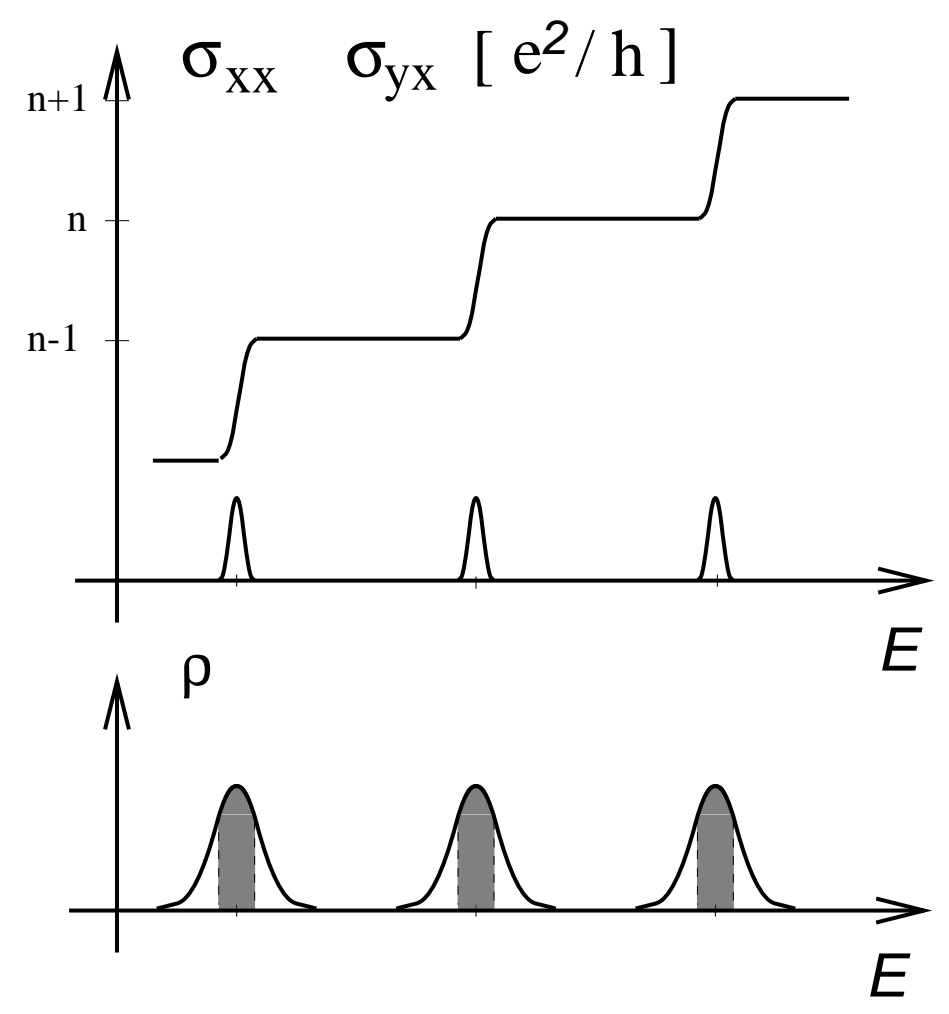




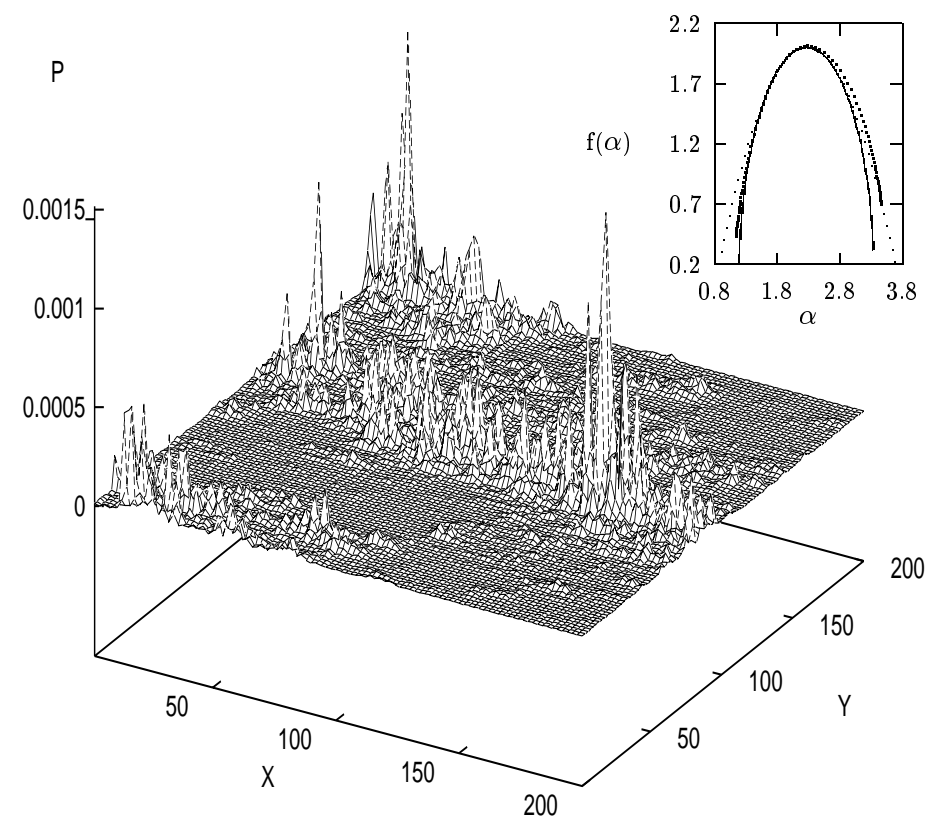




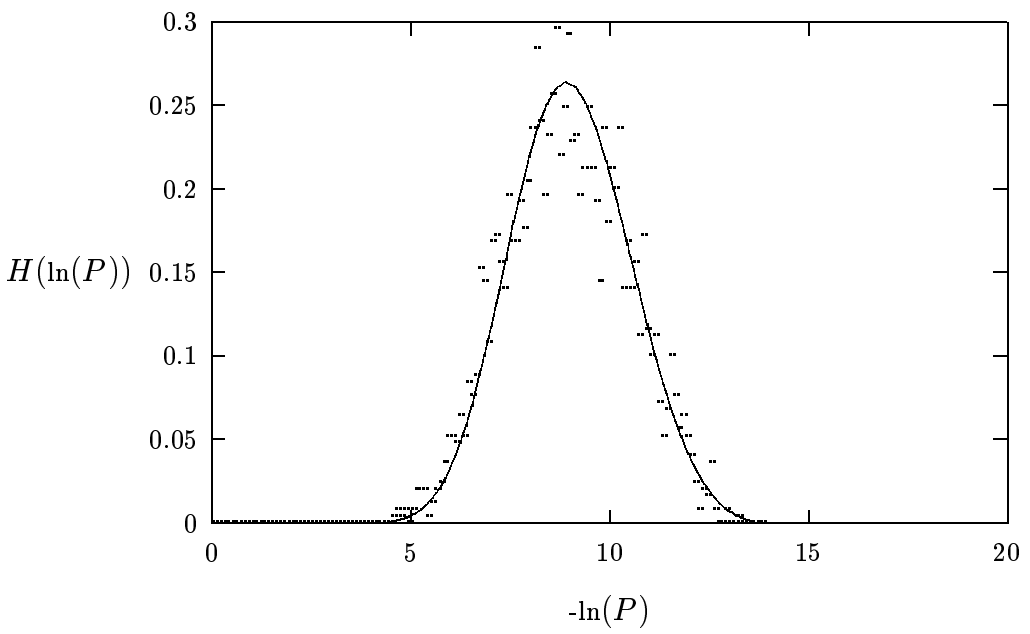




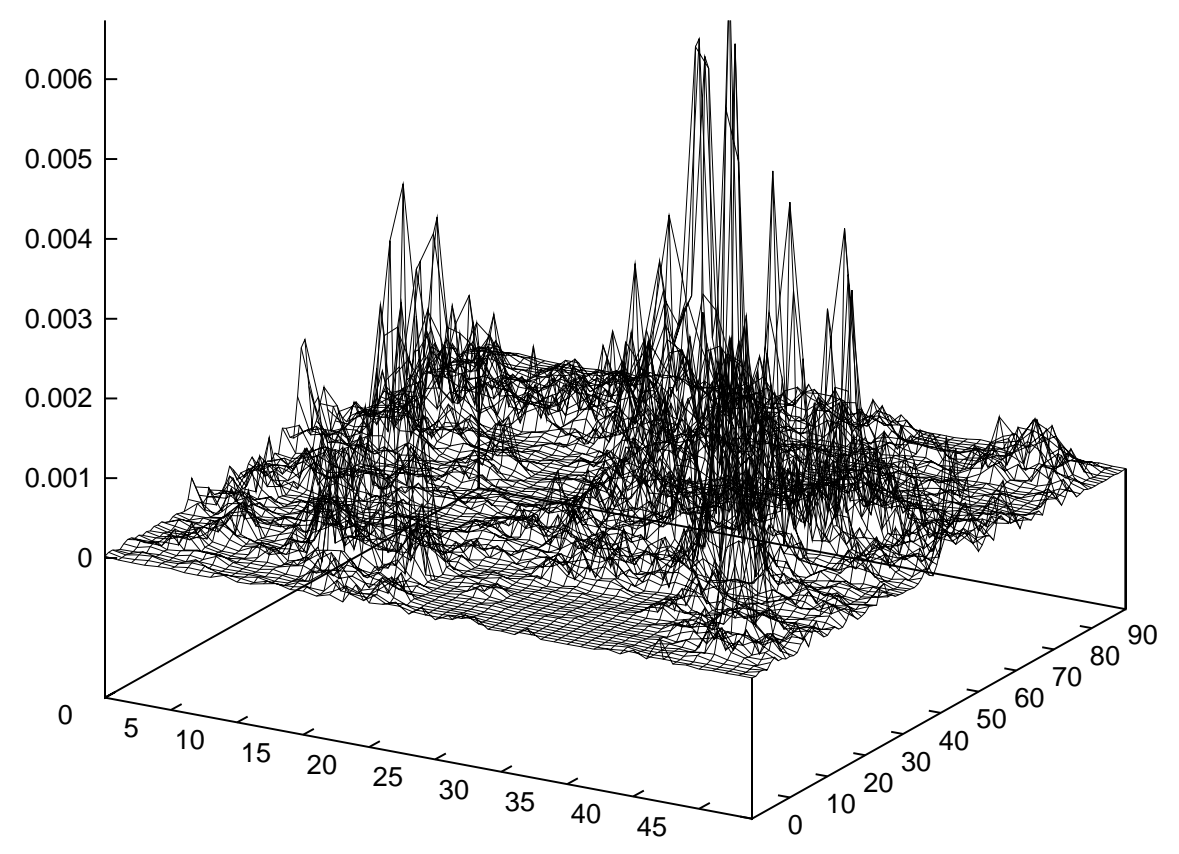




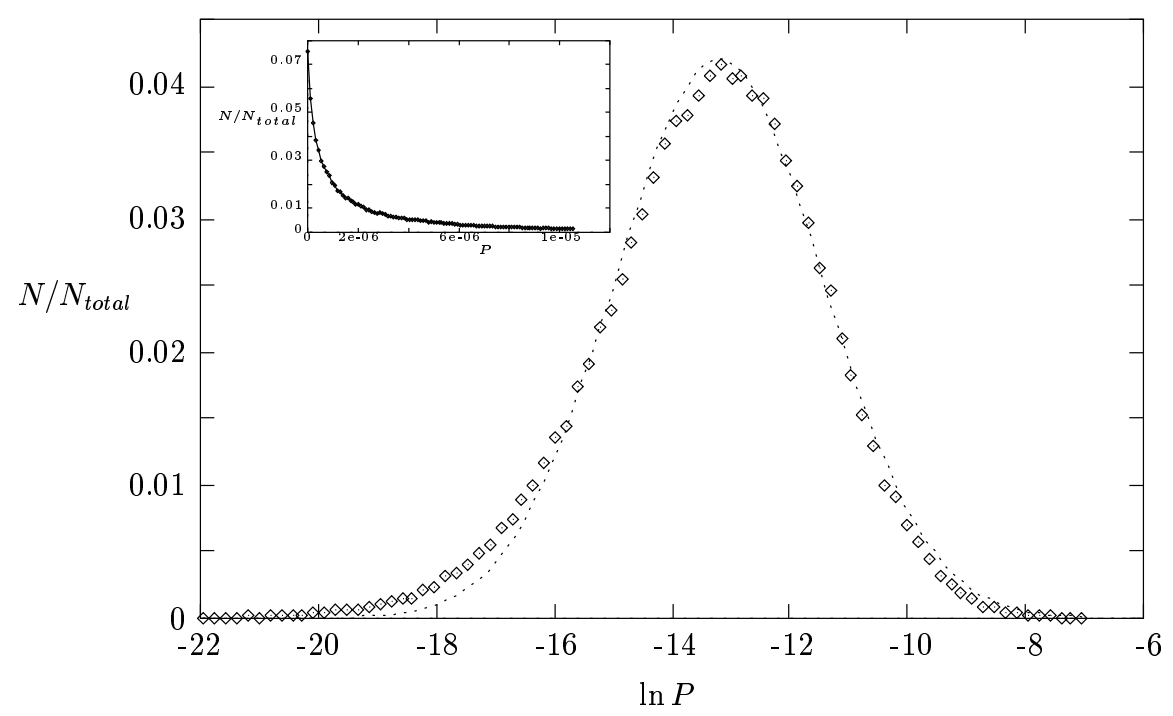




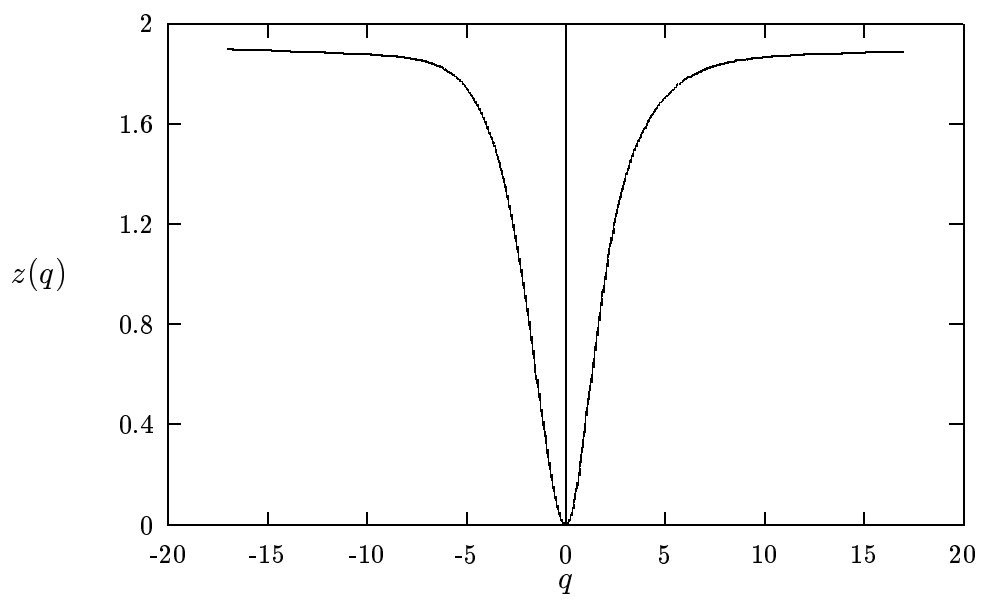




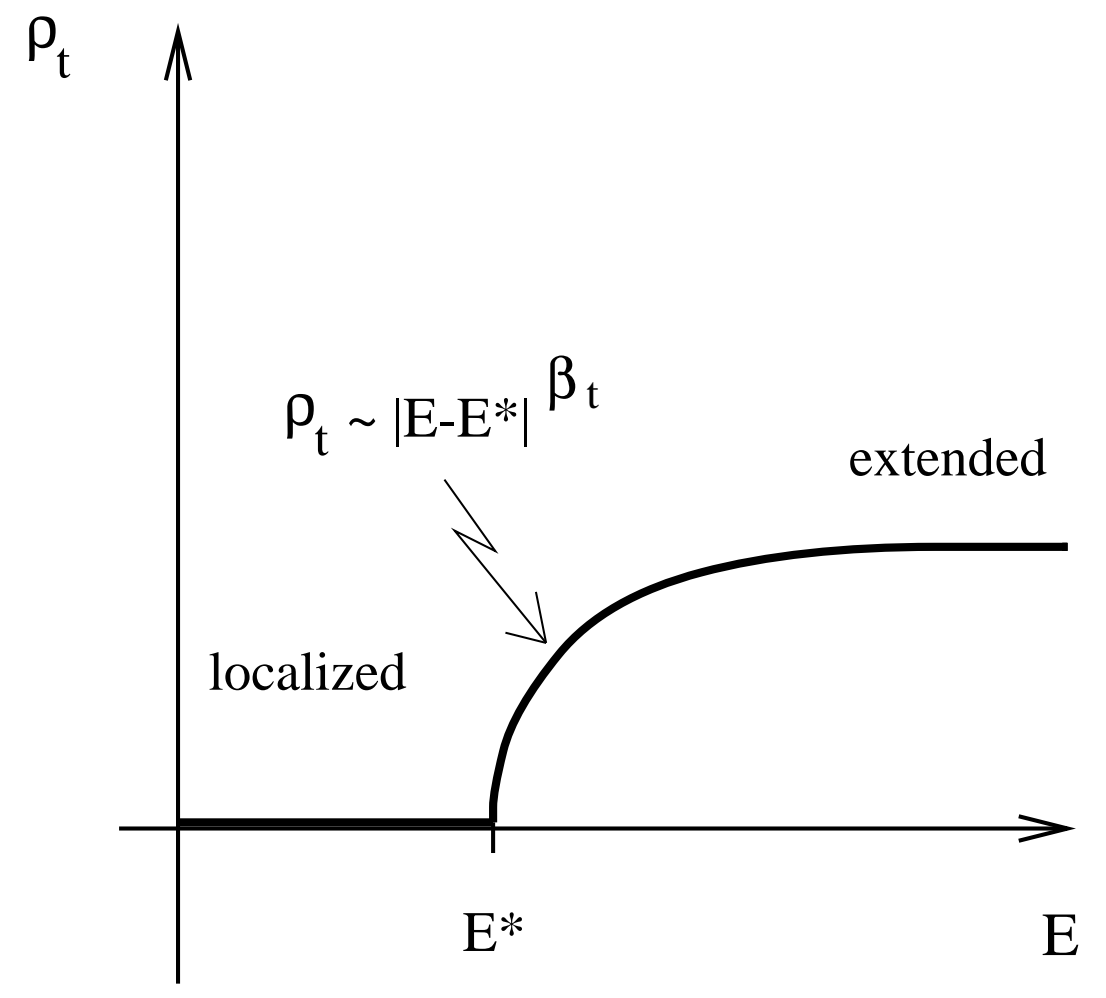

